\title{
PROPAGATION OF SINGULARITIES FOR THE WAVE EQUATION ON EDGE MANIFOLDS
}

\author{
RICHARD MELROSE, ANDRÁS VASY, AND JARED WUNSCH
}

\begin{abstract}
We investigate the geometric propagation and diffraction of singularities of solutions to the wave equation on manifolds with edge singularities. This class of manifolds includes, and is modelled on, the product of a smooth manifold and a cone over a compact fiber. Our main results are a general 'diffractive' theorem showing that the spreading of singularities at the edge only occurs along the fibers and a more refined 'geometric' theorem showing that for appropriately regular (nonfocusing) solutions, the main singularities can only propagate along geometrically determined rays. Thus, for the fundamental solution with initial pole sufficiently close to the edge, we are able to show that the regularity of the diffracted front is greater than that of the incident wave.
\end{abstract}

\section{Contents}

1. Introduction 2

1.1. Main Results 2

1.2. Previous results $\quad 7$

1.3. Proofs and plan of the paper 8

2. Edge manifolds 9

3. Calculi 12

4. Coisotropic regularity 15

5. Domains 19

6. Wavefront sets $(f>1) \quad 22$

7. Edge/b relationship 25

8. Ellipticity 31

9. Law of reflection 40

10. Fiber dimension $1 \quad 56$

11. Edge propagation $\quad 59$

12. Propagation of coisotropic regularity 61

13. Geometric theorem 63

14. Applications to Lagrangian data 65

Date: December 30, 2007.

The authors gratefully acknowledge financial support for this project, the first from the National Science Foundation under grant DMS-0408993, the second under grant DMS0201092, from a Clay Research Fellowship and a Sloan Fellowship and the third from the National Science Foundation under grants DMS-0401323 and DMS-0700318. They thank two anonymous referees for many suggestions that improved the exposition. 
Appendix: Iterative regularity under modules

\section{INTRODUCTION}

1.1. Main Results. In this paper, we investigate the geometric propagation and diffraction of singularities of solutions to the wave equation on manifolds with edge singularities. The main results are extension of those in [19] for the particular case of conic metrics, namely a general 'diffractive' theorem limiting the possible spreading of singularities at the boundary and a more refined 'geometric' theorem showing that for appropriately regular (nonfocusing) solutions the main singularities can only propagate along geometrically determined rays.

Let $X$ be an $n$-dimensional manifold with boundary, where the boundary, $\partial X$, is endowed with a fibration

$$
Z \rightarrow \partial X \stackrel{\pi_{0}}{\rightarrow} Y
$$

where $Y, Z$ are without boundary. Let $b$ and $f$ respectively denote the dimensions of $Y$ and $Z$ (the 'base' and the 'fiber'). By an edge metric $g$ on $X$ we shall mean a metric, $g$, on the interior of $X$ which is a smooth 2-cotensor up to the boundary but which degenerates there in a way compatible with the fibration. To state this condition precisely, consider the Lie algebra $\mathcal{V}(X)$ of smooth vector fields on $X$ which are tangent to the boundary, hence have well-defined restrictions to the boundary which are required to be tangent to the fibers of $\pi_{0}$. Let $x$ be a boundary defining function for $X$, then we require that $g$ be degenerate in the sense that

$$
g(V, V) \in x^{2} \mathcal{C}^{\infty}(X) \forall V \in \mathcal{V}(X) .
$$

This of course only fixes an 'upper bound' on $g$ near the boundary and we require the corresponding lower bound and also a special form of the leading part of the metric near the boundary. The lower bound is just the requirement that for any boundary point,

$$
\begin{aligned}
& V \in \mathcal{V}(X) \text { and } x^{-2} g(V, V)(p)=0 \\
& \quad \Longrightarrow V=\sum_{i} f_{i} V_{i}, V_{i} \in \mathcal{V}(X), f_{i} \in \mathcal{C}^{\infty}(X), f_{i}(p)=0 ;
\end{aligned}
$$

that is the vanishing of $g(V, V)$ to higher order than 2 at a boundary point means that $V$ vanishes at that point as an element of $\mathcal{V}(X)$. To capture the required special form near $\partial X$, consider within $\mathcal{V}(X)$ the subalgebra of vector fields which vanish at the boundary in the ordinary sense, we denote this Lie subalgebra

$$
\mathcal{V}_{0}(X) \subset \mathcal{V}(X)
$$


In addition to (1.1) we require that there be a product decomposition of a neighborhood of the boundary

$$
[0, \epsilon) \times \partial X \stackrel{p}{\underset{\sim}{\longrightarrow}} U \supset \partial X
$$

and a smooth metric $g_{0}$ (in the usual sense of extension across the boundary) on $[0, \epsilon) \times Y$ such that

$$
\begin{gathered}
\left(g-{\widetilde{\pi_{0}}}^{*} g_{0}\right)(V, W) \in x^{2} \mathcal{C}^{\infty}(X), \forall V, W \in \mathcal{V}(X) \text { and } \\
\left(g-{\widetilde{\pi_{0}}}^{*} g_{0}\right)(V, W) \in x^{3} \mathcal{C}^{\infty}(X), \quad \forall V \in \mathcal{V}_{0}(X), W \in \mathcal{V}(X) ;
\end{gathered}
$$

here $\widetilde{\pi_{0}}: U \longrightarrow[0, \epsilon) \times Y$ is the product extension of $\pi_{0}$ using (1.4).

We note that the metric $g_{0}$ on $[0, \epsilon) \times Y$ in (1.5) can always be brought to the product form

$$
g_{0}=d x^{2}+h(x)
$$

near $x=0$ for some boundary defining function $x$, with $h \in \mathcal{C}^{\infty}\left(\left[0, \epsilon^{\prime}\right) \times\right.$ $Y$; $\left.\operatorname{Sym}^{2} T^{*}([0, \epsilon) \times Y)\right), \epsilon^{\prime}>0$, i.e. $h(x)$ an $x$-dependent metric on $Y$. Thus the global restriction (1.5) means that in local coordinates $x, y, z$ near each boundary point,

$$
g=d x^{2}+h(x, y, d y)+x h^{\prime}(x, y, z, d x, d y)+x^{2} k(x, y, z, d x, d y, d z)
$$

where the $h^{\prime} \in \mathcal{C}^{\infty}\left(U ; \operatorname{Sym}^{2} T^{*}([0, \epsilon) \times Y)\right.$ and $k \in \mathcal{C}^{\infty}\left(U ; \operatorname{Sym}^{2} T^{*} X\right)$ and the restriction of $k$ to each fiber of the boundary is positive-definite. This latter condition is equivalent to (1.2).

A manifold with boundary equipped with such an edge metric will also be called an edge manifold or a manifold with edge structure. We draw the reader's particular attention to the two extreme cases: if $Z$ is a point, then an edge metric on $X$ is a simply a metric in the usual sense, smooth up to the boundary, while if $Y$ is a point, $X$ is a conic manifold (cf. [19]). A simple example of a more general edge metric is obtained by performing a real blowup on a submanifold $B$ of a smooth, boundaryless manifold $A$. The blowup operation simply introduces polar 'coordinates' near $B$, i.e. replaces $B$ by its spherical normal bundle, thus yielding a manifold $X$ with boundary. The pullback of a smooth metric on $A$ to $X$ is then an edge metric; here we have $Y=B$ and $F=S^{\operatorname{codim}(B)-1}$. A non-example that is nonetheless quite helpful in visualizing many of the constructions used in this paper is a manifold with a codimension-two corner, equipped with an incomplete metric: if we blow up the corner, we obtain a manifold of the type considered in this paper, but where $F$ is now a manifold with boundary, given by a closed interval representing an angular variable at the corner. More generally, any manifold with corners with an incomplete metric can be considered to have an iterated edge-structure: passing to polar coordinates near a corner yields an edge manifold with fiber given by another manifold with corners, more precisely the intersection of a sphere with an orthant. The authors intend to consider this situation in a subsequent paper. Note that in every case the 
boundary of $X$ is given geometrically by $Y$, as the fibers become metrically small as we approach the boundary.

Since we work principally with the wave equation, set $M=\mathbb{R} \times X$. Thus, the boundary of $M$ has a fibration with fiber $Z$ and base $\mathbb{R} \times Y$; it is an edge manifold with a metric of Lorentzian signature. We consider solutions $u$ to the wave equation

$$
\square u=\left(D_{t}^{2}-\Delta_{g}\right) u=0 \text { on } M
$$

with respect to this Lorentzian metric. In the simplest case, in which $X$ decomposes as the product $\mathbb{R}_{+} \times Y \times Z$ and the metric is a warped product,

$$
\square=D_{t}^{2}-\left(D_{x}^{2}+\frac{c}{x} D_{x}+\Delta_{Y}+\frac{1}{x^{2}} \Delta_{Z}\right) .
$$

In general the form of the operator is a little more complicated than this, but with similar leading part at the boundary.

We shall consider below only solutions of (1.8) lying in some 'finite energy space.' Thus, if $\mathcal{D}_{\alpha}$ is the domain of $\Delta^{\alpha / 2}$, where $\Delta$ is the Friedrichs extension from the space $\dot{\mathcal{C}}^{\infty}(X)$, of smooth functions vanishing to infinite order at the boundary, we require that a solution be admissible in the sense that it lies in $\mathcal{C}\left(\mathbb{R} ; \mathcal{D}_{\alpha}\right)$ for some $\alpha \in \mathbb{R}$.

In terms of adapted coordinates $x, y, z$ near a boundary point, an element of $\mathcal{V}(X)$ is locally an arbitrary smooth combination of the basis vector fields

$$
x \partial_{x}, x \partial_{y_{j}}, \partial_{z_{k}}
$$

and hence $\mathcal{V}(X)$ is equal to the space of all sections of a vector bundle (determined by $\left.\pi_{0}\right)$, which we call the edge tangent bundle and denote ${ }^{\mathrm{e}} T X$. This bundle is canonically isomorphic to the usual tangent bundle over the interior (and non-canonically isomorphic to it globally) with a well-defined bundle map ${ }^{\mathrm{e}} T X \longrightarrow T X$ which has rank $f$ over the boundary. Correspondingly, on $M$, the symbol of the wave operator extends to be of the form $x^{-2} p$ with $p$ a smooth function on the dual bundle to ${ }^{\mathrm{e}} T X$, called the edge cotangent bundle and denoted ${ }^{\mathrm{e}} T^{*} M$. The vector fields in (1.9), and $x \partial_{t}$, define linear functions $\tau, \xi, \eta$ and $\zeta$ on this bundle, in terms of which the canonical one-form on the cotangent bundle lifts under the dual map ${ }^{\mathrm{e}} T^{*} M \longrightarrow T^{*} M$ to the smooth section

$$
\tau \frac{d t}{x}+\xi \frac{d x}{x}+\eta \cdot \frac{d y}{x}+\zeta \cdot d z \text { of }{ }^{\mathrm{e}} T^{*} M .
$$

We will let ${ }^{\mathrm{e}} S^{*} M$ denote the unit cosphere bundle of e ${ }^{*} M$.

The characteristic variety of $\square$, i.e. the zero set of the symbol, will be interpreted as a subset $\Sigma \subset{ }^{\mathrm{e}} T^{*} M \backslash 0$; over the boundary it is given by the vanishing of $b$. Note that one effect of working on this 'compressed' cotangent bundle is that $\Sigma$ is smooth and is given in each fiber by the vanishing of a non-degenerate Lorentzian quadratic form.

The variables $\xi$ and $\zeta$ dual to $x$ and $z$ respectively play a different role to the duals of the base variables $(t, y)$. We will thus define a new bundle, 
denoted $\pi\left({ }^{\mathrm{e}} S^{*}(M)\right)$, whose sections are required to have $\xi=\zeta=0$ at $\partial M$. (For an explanation of the notation, see $\S 7$ ). Over $\partial M$, we may then decompose this bundle into elliptic, glancing, and hyperbolic sets much as in the usual case of manifolds with boundary:

$$
\pi\left({ }^{\mathrm{e}} S^{*} M\right)=\mathcal{E} \cup \mathcal{G} \cup \mathcal{H}
$$

where $\mathcal{G}$ is the light cone, and $\mathcal{E}$ its exterior, i.e. $\mathcal{G} \cup \mathcal{H}$ is the projection of the characteristic set to $\pi\left({ }^{\mathrm{e}} S^{*} M\right)$.

For each normalized point

$$
p=(\bar{t}, \bar{y}, \bar{z}, \bar{\tau}= \pm 1, \bar{\eta}) \in \mathcal{H},|\bar{\eta}|<1,
$$

it is shown below that there are two line segments of 'normal' null bicharacteristics in $\Sigma$, each ending at one of the two points above $p$ given by the solutions $\bar{\xi}$ of $\bar{\xi}^{2}+|\bar{\eta}|^{2}=1$. These will be denoted

$$
\mathcal{F}_{\bullet, p}
$$

where $\bullet$ is permitted to be $I$ or $O$, for 'incoming' or 'outgoing,' as $\operatorname{sgn} \bar{\xi}=$ $\pm \operatorname{sgn} \bar{\tau}(+$ for $I$ and - for $O)$. In the special case that the fibration and metric are of true product form

$$
d x^{2}+h(y, d y)+x^{2} k(z, d z)
$$

these bicharacteristics are simply

$$
\begin{gathered}
\mathcal{F}_{I, p}=\{t \leq \bar{t}, x=(\bar{t}-t)|\bar{\xi}|, y=y(t), z=\bar{z}, \tau=\bar{\tau}, \xi=\bar{\xi}, \eta=\eta(t), \zeta=0\} \\
\quad \text { and } \\
\mathcal{F}_{O, p}=\{t \geq \bar{t}, x=(t-\bar{t})|\bar{\xi}|, y=y(t), z=\bar{z}, \tau=\bar{\tau}, \xi=\bar{\xi}, \eta=\eta(t), \zeta=0\} ;
\end{gathered}
$$

where $(y(t), \eta(t))$ evolves along a geodesic in $Y$ which passes through $(\bar{y}, \bar{\eta})$ at time $t=\bar{t}$, and where $\bar{\tau}^{2}=\bar{\xi}^{2}+|\eta|^{2}=1$, and we have chosen the sign of $\bar{\xi}$ to agree/disagree with the sign of $\bar{\tau}$ in the incoming/outgoing cases.

As it is $Z$-invariant over the boundary, we may write $\mathcal{H}$ as the pull-back to $\partial M$ via $\pi_{0}$ of a corresponding set $\dot{\mathcal{H}}$. We may therefore consider all the bicharacteristic meeting the boundary in in a single fiber, with the same 'slow variables' $(t, y)$ and set

$$
\dot{\mathcal{F}}_{\bullet, q}=\bigcup_{p \in \pi_{0}^{-1}(q)} \mathcal{F}_{\bullet}, p, q \in \dot{\mathcal{H}} .
$$

These pencils of bicharacteristics touching the boundary at a given location in the 'slow' spacetime variables $(t, y)$, with given momenta in those variables, form smooth coisotropic (involutive) manifolds in the cotangent bundle near the boundary. The two main theorems of this paper show how microlocal singularities incoming along a bicharacteristic $\mathcal{F}_{I, p}$ are connected to those along bicharacteristics $\mathcal{F}_{O, p^{\prime}}$ for various values of $p, p^{\prime}$.

Our first main result is global in the fiber, and corresponds to the fact that the propagation of singularities is microlocalized in the slow variables $(t, y)$ and their duals while in general being global in the fiber. Let $\dot{\mathcal{F}}_{\bullet}^{\circ}$, 
denote the part of $\dot{\mathcal{F}}_{\bullet, q}$ over the interior $M^{\circ}$ of $M$ and in an appropriately small neighborhood of the boundary.

Theorem 1.1. For an admissible solution, $u$, to the wave equation and any $q \in \dot{\mathcal{H}}$,

$$
\dot{\mathcal{F}}_{I, q}^{\circ} \cap \mathrm{WF}^{k}(u)=\emptyset \Longrightarrow \dot{\mathcal{F}}_{O, q}^{\circ} \cap \mathrm{WF}^{k}(u)=\emptyset .
$$

Thus singularities interact with the boundary by specular reflection, preserving momentum in the slow variables. The lack of localization in the fibers is reflected by the fact that only the sets $\dot{\mathcal{F}}_{\bullet, q}$ rather than $\mathcal{F}_{\bullet}, p$ enter into the statement. Refinements of this result giving appropriate regularity at the boundary, not just in the interior, are discussed below.

We also prove a result regarding the behavior at glancing rays which touch the boundary at $\mathcal{G}$. In this case, singularities can propagate only along continuations of rays as generalized broken bicharacteristics much as in [18]. These curves are defined in $\S 7$ and in the non-glancing case are just unions of incoming and outgoing bicharacteristics associated to the same point in $\mathcal{H}$, while in the glancing case the definition is subtler. This allows Theorem 1.1 to be extended to the following concise statement.

Theorem 1.2. Singularities for admissible solutions propagate only along generalized broken bicharacteristics.

We also obtain a result which is microlocal in the fiber variable as well as in the slow variables but that necessarily has additional 'nonfocusing' hypotheses, directly generalizing that of [19], whose detailed explanation we postpone to $\S 12$. This condition, which is stated relative to a Sobolev space $H^{s}$, amounts to the requirement (away from, but near, the boundary) that the solution lie in the image of $H^{s}$ under the action of a sum of products of first-order pseudodifferential operators with symbols vanishing along $\dot{\mathcal{F}}_{I, q}$. Now consider two points $p, p^{\prime}$ in $\dot{\mathcal{H}}$ and lying above $q$, so in the same fiber $Z_{y}$. They are said to be geometrically related if they are the endpoints of a geodesic segment of length $\pi$ in $Z_{y}$.

Theorem 1.3. For an admissible solution u satisfying the nonfocusing condition relative to $H^{s}$ for $\dot{\mathcal{F}}_{I, q}^{\circ}, q \in \dot{\mathcal{H}}$, and for $p \in \mathcal{H}$ projecting to $q$,

$$
\begin{aligned}
& \mathcal{F}_{I, p^{\prime}}^{\circ} \cap \mathrm{WF}^{s}(u)=\emptyset \forall p^{\prime} \text { geometrically related to } p \Longrightarrow \\
& \mathcal{F}_{O, p}^{\circ} \cap \mathrm{WF}^{r}(u)=\emptyset \forall r<s .
\end{aligned}
$$

So, if we define 'geometric generalized bicharacteristics' as unions of bicharacteristic segments, entering and leaving $\partial M$ only at geometrically related points, then solutions satisfying an appropriate nonfocusing condition have the property that the strongest singularities propagate only along geometric generalized bicharacteristics. This may be visualized as follows: Consider an example in which only one singularity arrives at $\partial X$, propagating along $\mathcal{F}_{I, p^{\prime}}$ Theorem 1.3 shows that this singularity is diffracted into singularities which may emerge along the whole surface $\dot{\mathcal{F}}_{O, \pi_{0}\left(p^{\prime}\right)}$; these outgoing 
singularities are weaker than the incident singularity at all but the special family of geometrically related bicharacteristics. The geometric generalized bicharacteristics, along which stronger singularities can propagate, are precisely those which can be obtained locally as limits of families of bicharacteristics missing the edge entirely. In the special case in which $X$ is simply a smooth manifold $A$ blown up at a submanifold $B$, the geometric generalized bicharacteristics are just the lifts of bichacteristics in the usual sense. Condensation of singularities arguments and the uniqueness in Hörmander's propagation theorem show that, at least locally, full-strength singularities do indeed propagate along the geometric generalized bicharacteristics for some solutions.

The nonfocusing condition does hold for the forward fundamental solution with pole $o$ sufficiently near the boundary

$$
u_{o}(t)=\frac{\sin t \sqrt{\Delta}}{\sqrt{\Delta}} \delta_{o}
$$

We have $u_{o}(t) \in H_{\mathrm{loc}}^{s}(M)$ for all $s<-n / 2+1$, while the nonfocusing condition holds relative to $H^{s^{\prime}}$ for all $s^{\prime}<-n / 2+1+f / 2$. If $p \in \mathcal{H}$ is sufficiently close to $o$ and $\mathcal{F}_{I, p} \cap \mathrm{WF} U_{o}(t) \neq \emptyset$, so the incoming singularity strikes the boundary at $p$, then for each $p^{\prime}$ projecting to the same point in $\dot{\mathcal{H}}$ as $p$, but not geometrically related to it,

$$
\mathcal{F}_{O, p^{\prime}} \cap \mathrm{WF}^{r}(u)=\emptyset \forall r<-n / 2+1+f / 2 .
$$

Thus, the diffracted wave is almost $f / 2$ derivatives smoother than the primary singularities of the fundamental solution, so we obtain the following description of the structure of $u_{o}\left(X^{\circ}\right.$ will, as usual, denote the interior of $X)$ :

Corollary 1.4. For all $o \in X^{\circ}$ let $\mathcal{L}_{o}$ denote the flowout of $S N^{*}(\{o\})$ along bicharacteristics lying over $X^{\circ}$. If o is sufficiently close to $\partial X$, then for short time, the fundamental solution $u_{o}$ is a Lagrangian distribution along $\mathcal{L}_{o}$ lying in $H^{s}$ for all $s<-n / 2+1$ together with a diffracted wave, singular only at $\mathcal{F}_{O}$, that lies in $H^{r}$ for all $r<-n / 2+1+f / 2$, away from its intersection with $\mathcal{L}_{o}$.

The geometric generalized bicharacteristics in this case are just those at the intersection of the diffracted wave and $\mathcal{L}_{o}$.

1.2. Previous results. It has been known since the work of the first author [14] and Taylor [24] that $\mathcal{C}^{\infty}$-wavefront set of a solution to the wave equation on a manifold with concave boundary and Dirichlet boundary conditions does not propagate into the classical shadow region, that is, that wavefront set arriving tangent to the boundary does not 'stick to' the boundary, but rather continues past it. ${ }^{1}$ By contrast, diffractive effects have long been known to occur for propagation of singularities on more singular spaces.

\footnotetext{
${ }^{1}$ In the analytic category the contrary is the case - see [5], [21, 20, 22].
} 
The first rigorous example is due to Sommerfeld, [23], who analyzed the diffraction into the shadow region behind a straight edge in two dimensions. Many more such examples were studied by Friedlander [4], and a general analysis of the fundamental solution to the wave equation on product cones was carried out by Cheeger and Taylor $[2,3]$. Borovikov [1] has analyzed the structure of the fundamental solution on polyhedra. All of these works rely in an essential way on the technique of separation of variables, using the product structure of the cone. There is a quite general, but heuristic, geometric theory of diffraction due to Keller [10]. This has been confirmed in a few special cases.

Non-product situations and more singular manifolds have been less explored. Gérard and Lebeau in [6] explicitly analyzed the problem of an analytic conormal wave incident on an analytic corner in $\mathbb{R}^{2}$, obtaining a $1 / 2$-derivative improvement of the diffracted wavefront over the incident one, which is the analog of Theorem 1.3 above. Also in the analytic setting, Lebeau $[11,12]$ obtained a diffractive theorem analogous to Theorem 1.1 for a broad class of manifolds, including manifolds with corners; the second author [25] has recently obtained such a theorem in the $\mathcal{C}^{\infty}$ setting on manifolds with corners. As already noted, the first and third authors [19] have previously studied (non-product) conic manifolds in the $\mathcal{C}^{\infty}$ setting and obtained both Theorem 1.1 and Theorem 1.3 in that setting.

1.3. Proofs and plan of the paper. The proofs of Theorems 1.1 and 1.3 use two distinct but related pseudodifferential calculi. We have stated the results in terms of regularity measured by the edge calculus, which is best suited for studying the fine propagation of regularity into and out of the boundary along different bicharacteristics. For arguments that are global in the fiber, however, the $b$-calculus proves to be a better tool, and the proof of Theorem 1.1 is really a theorem about propagation of b-regularity, following the argument in the corners setting in [25]. Essential use is made of the fact that the product $x \xi$, with $\xi$ the the dual variable to $\partial_{x}$, is increasing along the bicharacteristic flow, and the test operators used are fiber constant so as not to incur the large error terms which would otherwise arise from commutation with $\Delta$.

By contrast, as in [19], the extension of the proof of Hörmander's propagation theorem for operators with real principal symbols to the edge calculus necessarily runs into the obstruction presented by manifolds of radial points, at which the Hamilton flow vanishes. The subprincipal terms then come into play, and propagation results are subject to the auxiliary hypothesis of divisibility. In particular, propagation results into and out of the boundary along bicharacteristics in the edge cotangent bundle up to a given Sobolev order are restricted by the largest power of $x$ by which $u$ is divisible, relative to the corresponding scale of edge Sobolev spaces. The divisibility given by energy conservation turns out to yield no useful information, as it yields a regularity for propagated singularities that is less than the regularity following 
directly from energy conservation. To prove Theorem 1.3 we initially settle for less information. As already noted, $\dot{\mathcal{F}}_{I, q}$ is a coisotropic submanifold of the cotangent bundle and as such 'coisotropic regularity' with respect to it may be defined in terms of iterated regularity under the application of pseudodifferential operators with symbols vanishing along $\dot{\mathcal{F}}_{I, \bullet}$. Thus, we begin by showing that coisotropic regularity in this sense, of any order, propagates through the boundary, with a fixed loss of derivatives. By interpolation with the results of Theorem 1.1 it follows that coisotropic regularity propagates through the boundary with epsilon derivative loss. Finally, imposing the nonfocusing condition allows Theorem 1.3 to be proved by a pairing argument, since this condition is a microlocal characterization of the dual to the space of distributions with coisotropic regularity.

\section{EDGE MANIFOLDS}

Let $X$ be an edge manifold, as defined in $\S 1.1$. In this section, we analyze the geodesic flow of $X$ in the edge cotangent bundle, and use this flow to describe a normal form for edge metrics.

As remarked above, by using the product decomposition near the boundary given by the distance along the normal geodesics in the base manifold $[0, \epsilon) \times Y$, we may write an edge metric in the form (1.7). The following result allows us to improve the form of the metric a bit further, so that $d x$ arises only in the $d x^{2}$ term:

Proposition 2.1. On an edge manifold, there exist choices for $x$ and for the product decomposition $U \equiv[0, \epsilon) \times Y$ such that

$$
g=d x^{2}+h(x, y, d y)+x h^{\prime}(x, y, z, d y)+x^{2} k(x, y, z, d y, d z)
$$

To prove Proposition 2.1 (and other results to come) it is natural to study the Hamilton flow associated to $g$ in the edge cotangent bundle of $X$. Let

$$
\alpha=\xi \frac{d x}{x}+\eta \cdot \frac{d y}{x}+\zeta \cdot d z
$$

denote the canonical one-form, lifted to ${ }^{\mathrm{e}} T^{*} X$. The dual basis of vector fields of ${ }^{\mathrm{e}} T X$ is $x \partial_{x}, x \partial_{y}, \partial_{z}$ and in terms of this basis the metric takes the form

$$
g=x^{2}\left(\begin{array}{ccc}
1 & O(x) & O(x) \\
O(x) & H+O(x) & O(x) \\
O(x) & O(x) & K
\end{array}\right)
$$

where $H$ and $K$ (which are nondegenerate) are defined respectively as the $d y \otimes d y$ and $d z \otimes d z$ parts of $h$ and $k$ at $x=0$. Here and henceforth we employ the convention that an $O\left(x^{k}\right)$ term denotes $x^{k}$ times a function in $\mathcal{C}^{\infty}(X)$.

Thus,

$$
g^{-1}=x^{-2}\left(\begin{array}{ccc}
1+O(x) & O(x) & O(x) \\
O(x) & H^{-1}+O(x) & O(x) \\
O(x) & O(x) & K^{-1}+O(x)
\end{array}\right)
$$


In fact, it is convenient to work on $M=X \times \mathbb{R}_{t}$, which has an induced boundary fibration, with base $Y \times \mathbb{R}_{t}$. Correspondingly, elements of ${ }^{\mathrm{e}} T^{*} M$ are written as

$$
\xi \frac{d x}{x}+\tau \frac{d t}{x}+\eta \cdot \frac{d y}{x}+\zeta \cdot d z
$$

so $(x, t, y, z, \xi, \tau, \eta, \zeta)$ are local coordinates on $M$. The canonical symplectic form on ${ }^{\mathrm{e}} T^{*} M^{\circ}$ is $d(\tau d t / x+\alpha)$, and the Hamilton vector field of the symbol of the wave operator $\square, p=(\tau / x)^{2}-g^{-1}$, is then $-2 x^{-2} H$, with

$$
\begin{gathered}
(2.2) \quad H=-\tau x \partial_{t}+(\tau \xi+O(x)) \partial_{\tau}+\left(x \xi+O\left(x^{2}\right)\right) \partial_{x} \\
+\left(\xi^{2}+\zeta_{i} \zeta_{j} K^{i j}+O(x)\right) \partial_{\xi}+\left(\zeta_{i} K^{i j}+O(x)\right) \partial_{z_{j}} \\
+\left(-\frac{1}{2} \zeta_{i} \zeta_{j} \frac{\partial K^{i j}}{\partial z_{k}}+O(x)\right) \partial_{\zeta_{k}}+\left(x \eta_{j} H^{i j}+O\left(x^{2}\right)\right) \partial_{y_{i}}+\left(\xi \eta_{i}+O(x)\right) \partial_{\eta_{i}} .
\end{gathered}
$$

(We employ the superscript $\circ$ to denote the interior of a manifold.)

Note that while it has not been emphasized in the expression above, this vector field is homogeneous of degree 1 in the fiber variables. We will be interested in the restriction of this flow to $\Sigma$, the characteristic variety of $\square$, where $(\tau / x)^{2}=g^{-1}$.

Lemma 2.2. Inside $\Sigma, H$ is radial, i.e. is tangent to the orbits of the fiber dilations on ${ }^{\mathrm{e}} T^{*} M \backslash 0$, if and only if $x=0$ and $\zeta=0$.

Proof. To be radial $H$ must be a multiple of $\xi \partial_{\xi}+\tau \partial_{\tau}+\eta \cdot \partial_{\eta}+\zeta \cdot \partial_{\zeta}$. In particular, in the expression above, the $\partial_{t}$ component must vanish, which inside $\Sigma$ implies $x=0$. The vanishing of the $\partial_{z}$ component further implies $\zeta=0$, and conversely $H$ is indeed radial at $x=0, \zeta=0$.

As usual, it is convenient to work with the cosphere bundle, ${ }^{\mathrm{e}} S^{*} M$, viewed as the boundary 'at infinity' of the radial compactification of e $T^{*} M$. Introducing the new variable

$$
\sigma=|\tau|^{-1}
$$

it follows that $H \sigma=-\xi \sigma+O(x)$. Setting

$$
\hat{\xi}=\xi \sigma, \hat{\eta}=\eta \sigma, \hat{\zeta}=\zeta \sigma
$$

we note that $\sigma \hat{\xi}, \hat{\eta}, \hat{\zeta}$ are coordinates on the fiber compactification of ${ }^{\mathrm{e}} T^{*} M \cap$ $\Sigma$, with $\sigma$ a defining function for ${ }^{\mathrm{e}} S^{*} M$ as the boundary at infinity. Then Lemma 2.2 becomes

Inside ${ }^{\mathrm{e}} S^{*} M \cap \Sigma, \sigma H$ vanishes exactly at $x=0, \hat{\zeta}=0$.

Let the linearization of $\sigma H$ at $q \in \Sigma \cap{ }^{\mathrm{e}} S^{*} M$ (where $x=0, \hat{\zeta}=0$ ) be $A_{q}$.

Lemma 2.3. For $q \in \dot{\mathcal{H}}$, i.e. such that $\hat{\xi}(q) \neq 0$, the eigenvalues of $A_{q}$ are $-\hat{\xi}, 0$ and $\hat{\xi}$, with $d x$ being an eigenvector of eigenvalue $\hat{\xi}$, and $d \sigma$ being an eigenvector of eigenvalue $-\hat{\xi}$. Moreover, modulo the span of $d x$, the $-\hat{\xi}$ eigenspace is spanned by $d \sigma$ and the $d \hat{\zeta}_{j}$, and the 0-eigenspace is spanned by $d t, d y_{j}, d \hat{\eta}_{j}, d \hat{\xi}$, and $d z_{j}+\hat{\xi}^{-1} \sum_{i} K^{i j}(q) d \hat{\zeta}_{i}$. 
Remark 2.4. This shows in particular that the span of the $d \hat{\zeta}_{j}$ (plus a suitable multiple of $d x$ ) is invariantly given as the stable/unstable eigenspace of $A_{q}$ inside $T_{q}^{* \mathrm{e}} S^{*} M$ according to $\hat{\xi}>0$ or $\hat{\xi}<0$. We denote this subspace of $T_{q}^{* \mathrm{e}} S^{*} M$ by $T_{q}^{*,-}\left({ }^{\mathrm{e}} S^{*} M\right)$.

Proof of Lemma 2.3. A straightforward calculation using (2.2) yields, in the coordinates $(x, y, t, z, \hat{\xi}, \hat{\eta}, \sigma, \hat{\zeta})$,

$$
\sigma H=\hat{\xi}\left(x \partial_{x}-\sigma \partial_{\sigma}-\hat{\zeta} \partial_{\hat{\zeta}}\right)+K^{i j} \hat{\zeta}_{i} \partial_{z_{j}}+K^{i j} \hat{\zeta}_{i} \hat{\zeta}_{j} \partial_{\hat{\xi}}-\frac{1}{2} \frac{\partial K^{i j}}{\partial z_{k}} \hat{\zeta}_{i} \hat{\zeta}_{j} \partial_{\hat{\zeta}_{k}}+x H^{\prime},
$$

with $H^{\prime}$ tangent to the boundary. Thus,

$$
\sigma H=\hat{\xi}\left(x \partial_{x}-\sigma \partial_{\sigma}-\hat{\zeta} \partial_{\hat{\zeta}}\right)+K^{i j} \hat{\zeta}_{i} \partial_{z_{j}}+x H^{\prime}+H^{\prime \prime},
$$

where $H^{\prime}$ is tangent to the boundary, and $H^{\prime \prime}$ vanishes quadratically at $q$ (as a smooth vector field). In particular, the linearization $A_{q}$ is independent of the $H^{\prime \prime}$ term, and is only affected by $x H^{\prime}$ as an operator with (at most) onedimensional range, lying in $\operatorname{sp}\{d x\}$. Moreover, $x H^{\prime} x$ vanishes quadratically at $\partial M$, so $d x$ is an eigenvector of $A_{q}$, and by the one-dimensional range observation, modulo $\operatorname{sp}\{d x\}$, the other eigenspaces can be read off from the form of $\sigma H$, proving the lemma.

Therefore, under the flow of $\sigma H$ we have

$$
\begin{aligned}
\hat{\zeta}^{\prime} & =-\hat{\xi} \hat{\zeta}+O\left(\hat{\zeta}^{2}\right)+O(x), \\
x^{\prime} & =\hat{\xi} x+O\left(x^{2}\right) .
\end{aligned}
$$

The Stable/Unstable Manifold Theorem can then be applied to the flow under $H$ and shows that for each $\left(y_{0}, z_{0}\right) \in \partial X, \xi_{0}, \eta_{0}$, with $\xi_{0} \neq 0$, there exists a unique bicharacteristic with the point $x=\hat{\zeta}=0,(y, z)=\left(y_{0}, z_{0}\right)$, $\hat{\xi}=\xi_{0}, \hat{\eta}=\eta_{0}$ in its closure and that a neighborhood of the boundary is foliated by such trajectories. Choosing $\xi_{0}=1, \hat{\eta}=0$ gives a foliation by normal trajectories arriving perpendicular to $\partial X$. Let $\tilde{x}$ be the distance to the boundary along the corresponding normal geodesics; it is a boundary defining function. Gauss's Lemma implies that in terms of the induced product decomposition the metric takes the form

$$
g=d \tilde{x}^{2}+h(\tilde{x}, \tilde{y}, d \tilde{y})+\tilde{x} h^{\prime}(\tilde{x}, \tilde{y}, \tilde{z}, d \tilde{y}, d \tilde{z})+\tilde{x}^{2} k(\tilde{x}, \tilde{y}, \tilde{z}, d \tilde{y}, d \tilde{z}) .
$$

This concludes the proof of Proposition 2.1.

This argument in fact gives a little more than Proposition 2.1, as we may take $\xi_{0} \neq 1$. In fact, for any $q=(t, y, z, \bar{\tau}, \bar{\xi}, \bar{\eta}) \in \mathcal{H}$ there exist two bicharacteristics having this point as its limit in the boundary, ${ }^{2}$ which we denote

$$
\mathcal{F}_{I / O, q}
$$

\footnotetext{
${ }^{2}$ The notation $\mathcal{H}, \dot{\mathcal{H}}$ will be further elucidated in $\S 7$.
} 
with the choice of $I / O$ determined by $\operatorname{sgn} \xi / \tau$. For $p=(t, y, \bar{\tau}, \bar{\xi}, \bar{\eta}) \in \dot{\mathcal{H}}$, we let

and

$$
\pi_{0}^{-1}(p)=\left\{(t, y, z, \bar{\tau}, \bar{\xi}, \bar{\eta}): z \in Z_{y}\right\}
$$

$$
\dot{\mathcal{F}}_{I / O, p}=\bigcup_{q \in \pi_{0}^{-1}(p)} \mathcal{F}_{I / O, p},
$$

and we collect all these bicharacteristics into

$$
\mathcal{F}_{I / O}=\bigcup_{p} \mathcal{F}_{I / O, p}, \quad \mathcal{F}=\mathcal{F}_{I} \cup \mathcal{F}_{O}
$$

For later use, we note that when the metric has been reduced to the normal form guaranteed by Proposition 2.1, the rescaled Hamilton vector field on ${ }^{\mathrm{e}} T^{*}(M)$ reduces to

$$
\begin{gathered}
H=-\tau x \partial_{t}+\tau \xi \partial_{\tau}+\xi x \partial_{x}+\left(\xi^{2}+|\zeta|_{\bar{K}}^{2}\right) \partial_{\xi}+\left(\zeta_{i} \bar{K}^{i j}+O(x)\right) \partial_{z_{j}} \\
+\left(-\frac{1}{2} \zeta_{i} \zeta_{j} \frac{\partial \bar{K}^{i j}}{\partial z_{k}}+O(x)\right) \partial_{\zeta_{k}}+\left(x \eta_{j} H^{i j}+O\left(x^{2}\right)\right) \partial_{y_{i}}+\left(\xi \eta_{i}+O(x)\right) \partial_{\eta_{i}}
\end{gathered}
$$

here we have let $\bar{K}^{i j}$ denote the whole of the $K^{-1}+O(x)$ term in the bottom right block of $g^{-1}$ as expressed in (2.1).

\section{Calculi}

In order to simplify the descriptions below, we assume that $M$ is compact. If $M$ is non-compact, we must insist on all operators having properly supported Schwartz kernels. For such operators all statements but the $L^{2}$-boundedness remain valid; when we restrict ourselves to operators with compactly supported Schwartz kernels, we have $L^{2}$-boundedness as well.

We use two algebras of pseudodifferential operators. Each arises from a Lie algebra $\mathcal{V}$ of smooth vector fields on $M$ tangent to $\partial M$, which is a $\mathcal{C}^{\infty}(M)$-module, and they have rather similar properties:

- For b-ps.d.o's, we take $\mathcal{V}=\mathcal{V}_{\mathrm{b}}(M)$, consisting of all $\mathcal{C}^{\infty}$ vector fields on $M$ that are tangent to $\partial M$.

- For edge ps.d.o's, $\partial M$ has a fibration $\phi: \partial M \rightarrow Y$, and we take $\mathcal{V}=\mathcal{V}_{\mathrm{e}}(M)$, consisting of all $\mathcal{C}^{\infty}$ vector fields on $M$ that are tangent to the fibers of $\phi$ (hence in particular to $\partial M$ ).

In terms of coordinates $x, y, z$ adapted to a local trivialization of the fibration on an edge manifold, with $x$ a boundary defining function and $z$ a coordinate in the fiber, it is easy to verify that $\mathcal{V}_{\mathrm{b}}(M)$ is spanned over $\mathcal{C}^{\infty}(M)$ by $x \partial_{x}$, $\partial_{y_{j}}, \partial_{z_{k}}$ while $\mathcal{V}_{\mathrm{e}}(M)$ is spanned over $\mathcal{C}^{\infty}(M)$ by $x \partial_{x}, x \partial_{y_{j}}, \partial_{z_{k}}$.

In both cases, $\mathcal{V}$ is the space of all smooth sections of a vector bundle $\mathcal{V}_{T M} \rightarrow M$. Thus, in the two cases, these bundles are denoted by ${ }^{\mathrm{b}} T M$ and ${ }^{\mathrm{e}} T M$ respectively; this notation extends in an obvious manner to all $\mathcal{V}$ objects we define below. The dual vector bundle is denoted by ${ }^{\mathcal{V}} T^{*} M$, and the corresponding cosphere bundle, i.e. $\left({ }^{\mathcal{V}} T^{*} M \backslash 0\right) / \mathbb{R}^{+}$is denoted by ${ }^{\mathcal{V}} S^{*} M$. As the space of all smooth vector fields on $M$ includes $\mathcal{V}$, there is a canonical 
bundle map ${ }^{\mathcal{V}} T M \rightarrow T M$, and a corresponding dual map, $T^{*} M \rightarrow{ }^{\mathcal{V}_{T}} T^{*}$. These are isomorphisms over the interior of $M$. Also, being a $\mathcal{C}^{\infty}$ section of $\mathcal{V}_{T M}$, every $V \in \mathcal{V}$ defines a linear functional on each fiber of ${ }^{\mathcal{V}} T^{*} M$. This is the principal symbol map:

$$
\mathcal{V}_{\sigma_{1}}: \mathcal{V} \rightarrow \mathcal{C}^{\infty} \text { fiber-linear functions on }{ }^{\mathcal{V}} T^{*} M
$$

We let $\operatorname{Diff}_{\mathcal{V}}^{k}(M)$ be the space of differential operators generated by $\mathcal{V}$ over $\mathcal{C}^{\infty}(M)$, so elements of $\operatorname{Diff}_{\mathcal{V}}^{k}(M)$ are finite sums of terms of the form $a V_{1} \ldots V_{l}, l \leq k, V_{j} \in \mathcal{V}, a \in \mathcal{C}^{\infty}(M)$. Thus, $\operatorname{Diff}_{\mathcal{V}}(M)=\bigcup_{k} \operatorname{Difff}_{\mathcal{V}}^{k}(M)$ is a filtered algebra over $\mathcal{C}^{\infty}(M)$. Defining $\mathcal{V}_{\sigma_{0}}(a)=\pi^{*} a$ for $a \in \mathcal{C}^{\infty}(M), \pi$ being the bundle projection ${ }^{\mathcal{V}_{T}} M \rightarrow M,{ }^{*}{ }_{\sigma}$ extends to a map

$$
\begin{aligned}
\mathcal{V}_{\sigma_{m}}: \operatorname{Diff}_{\mathcal{V}}^{m}(M) \longrightarrow & \longrightarrow \\
& \left\{\mathcal{C}^{\infty} \text { fiber-homogeneous polynomials of degree } m \text { on }{ }^{\mathcal{V}^{*}} T^{*}\right\},
\end{aligned}
$$

that is a filtered ring-homomorphism in the sense that

$$
\mathcal{V}_{\sigma_{m+l}}(A B)={ }^{\mathcal{V}} \sigma_{m}(A)^{\mathcal{V}} \sigma_{l}(B) \text { for } A \in \operatorname{Diff}_{\mathcal{V}}^{m}(M), B \in \operatorname{Diff}_{\mathcal{V}}^{l}(M) .
$$

We also fix a non-degenerate $b$-density $\nu$ on $M$, hence $\nu$ is of the form $x^{-1} \nu_{0}, \nu_{0}$ a non-degenerate $\mathcal{C}^{\infty}$ density on $M$, i.e. a nowhere-vanishing section of the density bundle $\Omega M:=\left|\wedge^{n}\right|(M)$. The density gives an inner product on $\dot{\mathcal{C}}^{\infty}(M)$. When below we refer to adjoints, we mean this relative to $\nu$, but the statements listed below not only do not depend on $\nu$ of the stated form, but would even hold for any non-degenerate density $x^{-l} \nu_{0}, \nu_{0}$ as above, $l$ arbitrary, as the statements listed below imply that conjugation by $x^{l}$ preserves the calculi.

For each of these spaces $\mathcal{V}$ there is an algebra of pseudodifferential operators, denoted by $\Psi_{\mathcal{V}}(M)$ which is a bifiltered $\mathcal{C}^{\infty}(M)$ *algebra of operators acting on $\dot{\mathcal{C}}^{\infty}(M), \Psi_{\mathcal{V}}(M)=\bigcup_{m, l} \Psi_{\mathcal{V}}^{m, l}(M)$, satisfying

(I) $\operatorname{Diff}_{\mathcal{V}}^{m}(M) \subset \Psi_{\mathcal{V}}^{m, 0}(M)$ (with the latter also denoted by $\Psi_{\mathcal{V}}^{m}(M)$ ),

(II) if $x$ is a boundary defining function of $M$ then $x^{l} \in \Psi_{\mathcal{V}}^{0, l}(M)$, and $x^{l} \Psi_{\mathcal{V}}^{m}(M)=\Psi_{\mathcal{V}}^{m, l}(M)$,

(III) the principal symbol map, already defined above for $\operatorname{Diff}_{\mathcal{V}}(M)$, extends to a bifiltered ${ }^{*}$-algebra homomorphism

$$
\mathcal{V}_{\sigma_{m, l}}: \Psi_{\mathcal{V}}^{m, l}(M) \rightarrow x^{l} S_{\text {hom }}^{m}\left({ }^{\mathcal{V}^{*}} T^{*} M \backslash 0\right)
$$

(IV) the principal symbol sequence is exact

$$
0 \rightarrow \Psi_{\mathcal{V}}^{m-1, l}(M) \hookrightarrow \Psi_{\mathcal{V}}^{m, l}(M) \rightarrow x^{l} S_{\text {hom }}^{m}\left({ }^{\mathcal{V}} T^{*} M \backslash 0\right) \rightarrow 0,
$$

$(\mathrm{V})$ for $A \in \Psi_{\mathcal{V}}^{m, l}(M), B \in \Psi_{\mathcal{V}}^{m^{\prime}, l^{\prime}}(M),[A, B] \in \Psi_{\mathcal{V}}^{m+m^{\prime}-1, l+l^{\prime}}(M)$ satisfies

$$
\mathcal{V}_{\sigma_{m+m^{\prime}-1, l+l^{\prime}}}([A, B])=\frac{1}{i}\left\{{ }^{\mathcal{V}} \sigma_{m, l}(A),{ }^{\mathcal{V}} \sigma_{m^{\prime}, l^{\prime}}(B)\right\},
$$


with the Poisson bracket defined a priori as a homogeneous function on $T^{*} M^{\circ} \backslash 0$, but extends to $x^{l+l^{\prime}}$ times a smooth function on ${ }^{\mathcal{V}} T^{*} M \backslash$ 0 ,

(VI) every $A \in \Psi_{\mathcal{V}}^{0,0}(M)$ extends from $\dot{\mathcal{C}}^{\infty}(M)$ by continuity to define a continuous linear map on $L^{2}(M)$, and for each $A \in \Psi_{\mathcal{V}}^{0,0}(M)$ there exists $A^{\prime} \in \Psi_{\mathcal{V}}^{-1,0}(M)$ such that for all $u \in L^{2}(M)$,

$$
\|A u\|_{L^{2}} \leq(2 \sup |a|)\|u\|_{L^{2}}+\left\|A^{\prime} u\right\|_{L^{2}},
$$

where $a=\mathcal{V}_{\sigma_{0,0}}(A)$.

In addition, there is an operator wave front set (or microsupport) $\mathrm{WF}_{\mathcal{V}}^{\prime}$ such that for $A \in \Psi_{\mathcal{V}}(M), \mathrm{WF}_{\mathcal{V}}^{\prime}(A) \subset{ }^{\mathcal{V}} S^{*} M$ is closed, and satisfies

(A) $\mathrm{WF}_{\mathcal{Y}}^{\prime}(A B) \subset \mathrm{WF}_{\mathcal{V}}^{\prime}(A) \cap \mathrm{WF}_{\mathcal{V}}^{\prime}(B)$,

(B) $\mathrm{WF}_{\mathcal{V}}^{\prime}(A+B) \subset \mathrm{WF}_{\mathcal{V}}^{\prime}(A) \cup \mathrm{WF}_{\mathcal{V}}^{\prime}(B)$,

(C) $\mathrm{WF}_{\mathcal{V}}^{\prime}(a)=\pi^{-1} \operatorname{supp} a$ for $a \in \mathcal{C}^{\infty}(M)$, where $\pi$ is the bundle projection,

(D) for any $K \subset{ }^{\mathcal{V}} S^{*} M$ closed and $U \subset{ }^{\mathcal{V}} S^{*} M$ open with $K \subset U$, there exists $A \in \Psi_{\mathcal{V}}^{0,0}(M)$ with $\mathrm{WF}_{\mathcal{V}}^{\prime}(A) \subset U$ and $\mathcal{V}_{\sigma_{0,0}}(A)=1$ on $K$ (so $\Psi_{\mathcal{V}}(M)$ is microlocal on $\left.{ }^{\mathcal{V}} S^{*} M\right)$,

(E) if $A \in \Psi_{\mathcal{V}}^{m, l}(M),{ }^{\mathcal{V}} \sigma_{m, l}(A)(q) \neq 0, q \in{ }^{\mathcal{V}} S^{*} M$, then there exists a microlocal parametrix $G \in \Psi_{\mathcal{V}}^{-m,-l}(M)$ such that

$$
q \notin \mathrm{WF}_{\mathcal{V}}^{\prime}(G A-\mathrm{Id}), q \notin \mathrm{WF}_{\mathcal{V}}^{\prime}(A G-\mathrm{Id}),
$$

(F) if $A \in \Psi_{\mathcal{V}}^{m, l}(M)$ and $\mathrm{WF}_{\mathcal{V}}^{\prime}(A)=\emptyset$ then $A \in \Psi_{\mathcal{V}}^{-\infty, l}=\bigcap_{m^{\prime}} \Psi_{\mathcal{V}}^{m^{\prime}, l}$ - thus, $\mathrm{WF}_{\mathcal{V}}^{\prime}$ captures $A$ modulo operators of order $-\infty$ in the smoothing sense, just as ${ }^{\mathcal{V}} \sigma_{m, l}$ captures $A$ modulo operators of order $(m-1, l)$.

Note that if $b \in x^{l} S_{\text {hom }}^{m}\left(\mathcal{V}^{*} M \backslash 0\right)$ then for any $U \subset{ }^{\mathcal{V}} S^{*} M$ open satisfying $U \supset \operatorname{supp} b$ there exists $B \in \Psi_{\mathcal{V}}^{m, l}(M)$ with $\operatorname{WF}_{\mathcal{V}}^{\prime}(B) \subset U$ and $\mathcal{V}_{\sigma_{m, l}}(B)=b$. Indeed, with $K=\operatorname{supp} b$, let $A \in \Psi_{\mathcal{V}}^{0,0}(M)$ be as in (D) above, so $\mathrm{WF}_{\mathcal{V}}^{\prime}(A) \subset$ $U$ and $\mathcal{V}_{\sigma_{0,0}}(A)=1$ on $K$. By the exactness of the symbol sequence, there is $B_{0} \in \Psi_{\mathcal{V}}^{m, l}(M)$ and $\mathcal{V}_{\sigma_{m, l}}(B)=b$. Then $B=A B_{0}$ satisfies all requirements.

Beyond these rather generic properties, shared by the b- and edge-calculi, we will use one further, more specialized property of the b-calculus: if $V \in$ $\mathcal{V}_{\mathrm{b}}(M)$ is such that $\left.V\right|_{\partial M}=x D_{x}$ (restriction in the sense of $\mathcal{V}_{\mathrm{b}}(M)$, i.e. as sections of $\left.{ }^{\mathrm{b}} T M\right)$, then for $A \in \Psi_{\mathrm{b}}^{m}(M),[A, V] \in x \Psi_{\mathrm{b}}^{m}(M)$, i.e. there is a gain of $x$ over the a priori statements. (Note that $x \partial_{x}$, the 'radial vector field', is a well-defined section of ${ }^{\mathrm{b}} T M$ at $\partial M$.)

The above property in fact follows from the analysis of the normal operator associated to $V$. More generally, for a differential operator in the $b$-calculus, we obtain a model, or normal operator by freezing coefficients of b-vector fields at the boundary: in coordinates $(x, w) \in \mathbb{R}_{+} \times \mathbb{R}^{k}$ with $x$ a 
boundary defining function, if

$$
P=\sum a_{i, \alpha}(x, w)\left(x D_{x}\right)^{i} D_{w}^{\alpha}
$$

then

$$
N(P)=\sum a_{i, \alpha}(0, w)\left(x D_{x}\right)^{i} D_{w}^{\alpha}
$$

is now an operator on $\mathbb{R}_{+} \times \partial M$. Likewise, if $Q$ is an edge operator given by

$$
Q=\sum b_{i, \alpha, \beta}(x, y, z)\left(x D_{x}\right)^{i}\left(x D_{y}\right)^{\alpha} D_{z}^{\beta}
$$

on a manifold with fibered boundary then

$$
N(Q)=\sum b_{i, \alpha, \beta}(0, y, z)\left(x D_{x}\right)^{i}\left(x D_{y}\right)^{\alpha} D_{z}^{\beta}
$$

is an operator on $\mathbb{R}_{+}^{b+1} \times F$, where $F$ is the fiber and $b$ the dimension of the base of the boundary fibration. These normal operators are in fact homomorphisms from the respective algebras, and they extend to act on the algebras $\Psi_{\mathcal{V}}^{m}(M)$. Their principal utility is the following:

$$
A \in x \Psi_{\mathcal{V}}^{m}(M) \Longleftrightarrow N(A)=0 .
$$

We will make very little use of the normal operators; for more detailed discussion, see [13].

We will also require a conormal variant of the b-calculus described above, where polyhomogeneous symbols $\left(S_{\mathrm{hom}}^{m}\right)$ are replaced by those satisfying Kohn-Nirenberg type symbol estimates $\left(S^{m}\right)$. We denote this calculus $\Psi_{\mathrm{b} \infty}$. It satisfies all the properties above, except that the symbol map now takes values in $S^{m}\left({ }^{\mathrm{b}} T^{*} M\right) / S^{m-1}\left({ }^{\mathrm{b}} T^{*} M\right)$. This calculus will be important in making approximation arguments in our b-calculus-based positive commutator estimates.

A unified treatment of the two calculi discussed in this section can be found in Mazzeo [13]. This was the original treatment of the edge calculus, while the $b$-calculus originates with the work of the first author [15].

\section{Coisotropic Regularity}

Theorem 4.1. Away from glancing rays, the set $\mathcal{F}$ is a coisotropic submanifold of the symplectic manifold ${ }^{\mathrm{e}} T^{*} M$, i.e. contains its symplectic orthocomplement.

Proof. We split $\mathcal{F}$ into its components $\mathcal{F}_{I}$ and $\mathcal{F}_{O}$; by symmetry, it suffices to work on $\mathcal{F}_{I}$.

It suffices to exhibit a set of functions $\alpha_{i}$ vanishing on $\mathcal{F}_{I}$ whose differentials yield a basis of sections of $N^{*} \mathcal{F}_{I}$ such that the Poisson bracket of any two also vanishes on $\mathcal{F}_{I}$.

To begin, we let $H_{p_{0}}$ denote the Hamilton vector field of $p_{0}={ }^{\mathrm{e}} \sigma_{2,-2}(\square)$ (and more generally, $H_{q}$ the Hamilton vector field of any symbol $q$ ); let $\varphi=x^{2} /|\tau|$ so $\varphi H_{p_{0}} \in \mathcal{V}_{b}\left({ }^{\mathrm{e}} T^{*}(M) \backslash 0\right)$, and is homogeneous of degree zero. The vector field $\varphi H_{p_{0}}$ is not itself a Hamilton vector field: we have

$$
\varphi H_{p_{0}}=H_{\varphi p_{0}}-p_{0} H_{\varphi} \text {. }
$$


This last vector field has the virtue of vanishing on $\mathcal{F}_{I}$, however.

Now $\mathcal{F}_{I}$ is a conic submanifold of ${ }^{\mathrm{e}} T^{*} M$, hence has a set of defining functions that are homogeneous of degree 0 . Letting $\hat{\zeta}_{i}=\zeta_{i} /|\tau|$ we note that it follows from (2.3) that we may take defining functions for $\mathcal{F}_{I}$ to be homogeneous functions of degree zero of the form

$$
\alpha_{i}=\hat{\zeta}_{i}+O(x), \quad i=1, \ldots, f,
$$

with $d \alpha_{i}(q)$ an eigenvector of the linearization $A_{q}$ of $\varphi H_{p_{0}}$ of eigenvalue $-\hat{\xi}$, plus one extra to keep us in the characteristic set: $\alpha_{0}=x^{2} p_{0} /|\tau|=\varphi p_{0}$. We immediately note that for $i \geq 1$, we have

$$
\left\{\alpha_{0}, \alpha_{i}\right\}=\varphi H_{p_{0}} \alpha_{i}+p_{0} H_{\varphi} \alpha_{i}=p_{0} H_{\varphi}\left(\alpha_{i}\right)
$$

which is a smooth multiple of $p_{0}$, so that Poisson brackets with $\alpha_{0}$ never present difficulties. It remains to show that $\left\{\alpha_{i}, \alpha_{j}\right\}$ is in the span of the $\alpha_{i}$ 's for $i=1, \ldots, f$.

The Jacobi identity yields

$$
H_{\varphi p_{0}}\left\{x \alpha_{i}, x \alpha_{j}\right\}=\left\{H_{\varphi p_{0}}\left(x \alpha_{i}\right), x \alpha_{j}\right\}-\left\{H_{\varphi p_{0}}\left(x \alpha_{j}\right), x \alpha_{i}\right\}, \quad i, j=1, \ldots, f .
$$

Using (4.1) and the tangency of $\varphi H_{p_{0}}$ to $\mathcal{F}_{I}$ and to $x=0$ we find that $H_{\varphi p_{0}}\left(x \alpha_{i}\right)$ is a smooth function vanishing at both $x=0$ and on $\mathcal{F}_{I}$, hence we have

$$
H_{\varphi p_{0}}\left(x \alpha_{i}\right)=\sum b_{i k}^{\prime} x \alpha_{k}
$$

where $b_{i k}^{\prime} \in \mathcal{C}^{\infty}$. We can in fact say a little more: since $\varphi H_{p_{0}}$ is a smooth vector field tangent both to $x=0$ and to $\mathcal{F}_{I}$, and as $d \alpha_{i}(q)$, resp. $d x$ are eigenvectors of the linearization of $\varphi H_{p_{0}}$ at $\partial \mathcal{F}_{I}$ with eigenvalue $-\hat{\xi}$, resp. $\hat{\xi}$, we have

$$
\varphi H_{p_{0}} x=\hat{\xi} x+x^{2} b+\sum c_{j} x \alpha_{j}, \varphi H_{p_{0}} \alpha_{i}=-\hat{\xi} \alpha_{i}+x \sum r_{i j} \alpha_{j}+r_{i},
$$

with $b, c_{i}, r_{i j}, r_{j}$ all smooth, $r_{i}$ vanishing quadratically at $\mathcal{F}_{I}$. Thus,

$$
\varphi H_{p_{0}}\left(x \alpha_{i}\right)=\sum b_{i k}\left(x^{2} \alpha_{k}\right)+r_{i}^{\prime}
$$

for smooth functions $b_{i k}$ and $r_{i}^{\prime}$ and with $r_{i}^{\prime}$ quadratically vanishing on $\mathcal{F}_{I}$.

Observe now that $p_{0} H_{\varphi}\left(x \alpha_{i}\right)=\left(\varphi p_{0}\right) x c_{i}$ with $c_{i}$ smooth, and $\left\{\left(\varphi p_{0}\right) x c_{i}, x \alpha_{j}\right\}$ vanishes at $p_{0}=0$ (cf. (4.2)). As a result, by (4.1) and (4.3), we see that

$$
\varphi H_{p_{0}}\left\{x \alpha_{i}, x \alpha_{j}\right\}=\sum x c_{i j k l}\left\{x \alpha_{k}, x \alpha_{l}\right\}+r
$$

where $r$ is a smooth term vanishing on $\mathcal{F}_{I}$.

Now we restrict to $\mathcal{F}_{I}$. By construction of $\mathcal{F}_{I}$, the vector field $\varphi H_{p_{0}}$ vanishes identically at $\mathcal{F}_{I} \cap \partial M$, hence it is divisible by a factor of $x$ when restricted to $\mathcal{F}_{I}$, with the quotient being a vector field on $\mathcal{F}_{I}$ transverse to $\partial \mathcal{F}_{I}$. Thus if we let $s$ be a parameter along the flow generated by $\varphi H_{p_{0}}$, with $s=0$ at $\partial \mathcal{F}_{I}$, we have with $c_{i j k l}^{\prime}$ smooth,

$$
\left.\frac{d}{d s}\left\{x \alpha_{i}, x \alpha_{j}\right\}\right|_{\mathcal{F}_{I}}=\left.\sum c_{i j k l}^{\prime}\left\{x \alpha_{k}, x \alpha_{l}\right\}\right|_{\mathcal{F}_{I}}
$$


Now $x \alpha_{k}$ and $x \alpha_{l}$ Poisson commute at $\partial M$, so we conclude that $\left\{x \alpha_{i}, x \alpha_{j}\right\}=$ 0 on $\mathcal{F}_{I}$.

This implies that in fact $\left\{\alpha_{i}, \alpha_{j}\right\}=0$ on $\mathcal{F}_{I}$, as $\left\{x \alpha_{i}, x \alpha_{j}\right\}-x^{2}\left\{\alpha_{i}, \alpha_{j}\right\}$ also vanishes there.

We now fix an arbitrary open set $U \subset{ }^{\mathrm{e}} T^{*} M$ disjoint from rays meeting $x=\xi=0$. We omit the set $U$ from the notation below, by abuse of notation.

\section{Definition 4.2 .}

(a) Let $\Psi_{\mathrm{e}}(U)$ be the subset of $\Psi_{\mathrm{e}}(M)$ consisting of operators $A$ with $\mathrm{WF}_{\mathrm{e}}{ }^{\prime} A \subset U$.

(b) Let $\mathcal{M}$ denote the module of pseudodifferential operators in $\Psi_{\mathrm{e}}^{1}(M)$ given by

$$
\mathcal{M}=\left\{A \in \Psi_{\mathrm{e}}^{1}(U):\left.\sigma(A)\right|_{\dot{\mathcal{F}}}=0\right\} .
$$

(c) Let $\mathcal{A}$ be the algebra generated by $\mathcal{M}$, with $\mathcal{A}^{k}=\mathcal{A} \cap \Psi_{\mathrm{e}}^{k}(M)$. Let $\mathrm{H}$ be a Hilbert space on which $\Psi_{\mathrm{e}}^{0}(M)$ acts, and let $K \subset{ }^{\mathrm{e}} T^{*}(M)$ be a conic set.

(d) We say that $u$ has coisotropic regularity of order $k$ relative to $\mathrm{H}$ in $K$ if there exists $A \in \Psi_{\mathrm{e}}^{0}(M)$, elliptic on $K$, such that $\mathcal{A}^{k} A u \subset \mathrm{H}$.

(e) We say that $u$ satisfies the nonfocusing condition of degree $k$ relative to $\mathrm{H}$ on $K$ if there exists $A \in \Psi_{\mathrm{e}}^{0}(M)$, elliptic on $K$, such that $A u \subset \mathcal{A}^{k} \mathrm{H}$. We say that $u$ satisfies the nonfocusing condition relative to $\mathrm{H}$ on $K$ if it satisfies the condition to some degree.

Remark 4.3. The conditions of coisotropic regularity and nonfocusing are dual to one another, at least in a microlocal sense: if $u$ satisfies (d) and $v$ satisfies (e) then the $L^{2}$ pairing of $A u$ and $A v$ makes sense.

Lemma 4.4. The module $\mathcal{M}$ is a test module in a sense analogous to [7, Definition 6.1]. That is, $\mathcal{M}$ is closed under commutators and is finitely generated in the sense that there exist finitely many $A_{i} \in \Psi_{\mathrm{e}}^{1}(M), i=$ $0,1, \ldots, N, A_{0}=\mathrm{Id}$, such that

$$
\mathcal{M}=\left\{A \in \Psi_{\mathrm{e}}^{1}(U): \exists Q_{i} \in \Psi_{\mathrm{e}}^{0}(U), A=\sum_{j=0}^{N} Q_{i} A_{i}\right\}
$$

Moreover, we may take $A_{N}$ to have symbol $|\tau| a_{N}=x^{2}|\tau|^{-1} p, p=\sigma(\square)$, and $A_{i}, i=1, \ldots, N-1$ to have symbol $|\tau| a_{i}$ with $d a_{i}(q) \in T_{q}^{*,-}\left({ }^{\mathrm{e}} S^{*} M\right)$ for $q \in \partial \dot{\mathcal{F}}$, where we used the notation of Remark 2.4.

Proof. For $A, B \in \mathcal{M},[A, B] \in \Psi_{\mathrm{e}}^{1}(M), \mathrm{WF}_{\mathrm{e}}{ }^{\prime}([A, B]) \subset U$ and $\sigma([A, B])=$ $\frac{1}{i}\{\sigma(A), \sigma(B)\}$, so $\left.\sigma([A, B])\right|_{\mathcal{F}}=0$ as $\dot{\mathcal{F}}$ is coisotropic. Thus, $\mathcal{M}$ is closed under commutators.

Let $a_{i}, i=1, \ldots, N$, be smooth homogeneous degree 0 functions on ${ }^{\mathrm{e}} T^{*} M \backslash 0$ such that $a_{i}$ vanish on $\dot{\mathcal{F}}$ and $d a_{i}(q), i=1, \ldots, N$, spans $N_{q}^{*} \dot{\mathcal{F}}$ for each $q \in \dot{\mathcal{F}}$. In particular, we may take $a_{N}=x^{2}|\tau|^{-1} p_{0}=\varphi p_{0}$ and choose $a_{i}$, $i=1, \ldots, N-1$ as in the statement of the lemma. Let $A_{i}$ be any elements 
of $\Psi_{\mathrm{e}}^{1}(M)$ with $\sigma\left(A_{i}\right)=|\tau| a_{i}$. In view of this spanning property, every homogeneous degree 1 function $a \in \mathcal{C}^{\infty}\left({ }^{\mathrm{e}} T^{*} M \backslash 0\right)$ with supp $a \subset U$ and $\left.a\right|_{\dot{\mathcal{F}}}=0$ can be written as $\sum_{i=1}^{N} q_{i}|\tau| a_{i}$ with $\operatorname{supp} q_{i} \subset U, q_{i}$ smooth homogeneous degree 0 , so if $Q_{i} \in \Psi_{\mathrm{e}}^{0}(M)$ are chosen to satisfy $\mathrm{WF}_{\mathrm{e}}{ }^{\prime}\left(Q_{i}\right) \subset U$ and $\sigma\left(Q_{i}\right)=q_{i}$, then $Q_{0}=A-\sum_{i=1}^{N} Q_{i} A_{i}$ satisfies $\mathrm{WF}_{\mathrm{e}}{ }^{\prime}(Q) \subset U, Q_{0} \in \Psi_{\mathrm{e}}^{0}(M)$, proving the lemma.

We recall from [7]:

Lemma 4.5. (See [7, Lemma 6.3].) If $A_{i}, 0 \leq i \leq N$, are generators for $\mathcal{M}$ in the sense of Lemma 4.4 with $A_{0}=\mathrm{Id}$, then

$$
\mathcal{A}^{k}=\left\{\sum_{|\alpha| \leq k} Q_{\alpha} \prod_{i=1}^{N} A_{i}^{\alpha_{i}}, Q_{\alpha} \in \Psi_{\mathrm{e}}^{0}(U)\right\}
$$

where $\alpha$ runs over multiindices $\alpha:\{1, \ldots, N\} \rightarrow \mathbb{N}_{0}$ and $|\alpha|=\alpha_{1}+\cdots+\alpha_{N}$.

Remark 4.6. The notation here is that the empty product is $A_{0}=\mathrm{Id}$, and the product is ordered by ascending indices $A_{i}$. The lemma is an immediate consequence of $\mathcal{M}$ being both a Lie algebra and a module; the point being that products may be freely rearranged, modulo terms in $\mathcal{A}^{k-1}$.

Now, $A_{N}$ being a multiple of $\square$, modulo $\Psi_{\mathrm{e}}^{0}(M)$, regularity under summands $Q_{\alpha} \prod_{i=1}^{N} A_{i}^{\alpha_{i}}$ with $\alpha_{N} \neq 0$ and $|\alpha|=k$ is automatic for solutions of $\square u=0$, once regularity with respect to $\mathcal{A}^{k-1}$ is known. The key additional information we need is:

Lemma 4.7. For $l=1, \ldots, N-1$,

$$
x^{2} i\left[A_{l}, \square\right]=\sum_{j=0}^{N} C_{l j} A_{j}, C_{l j} \in \Psi_{\mathrm{e}}^{1}(M),\left.\sigma\left(C_{l j}\right)\right|_{\partial \dot{\mathcal{F}}}=0 \text { for } j \neq 0 .
$$

Remark 4.8. In fact, it would suffice to have $\left.\sigma\left(C_{l j}\right)\right|_{\partial \dot{\mathcal{F}}}$ diagonal with

$$
\left.\operatorname{Re} \sigma\left(C_{l l}\right)\right|_{\partial \dot{\mathcal{F}}} \geq 0
$$

as in [7, Equation (6.14)] or an even weaker lower (or upper) triangular statement, see [7, Section 6].

Proof. Let $|\tau| a_{l}=\sigma\left(A_{l}\right)$, considered as a homogeneous degree 1 function on ${ }^{\mathrm{e}} T^{*} M$. Note that $a_{N}=\sigma\left(A_{N}\right)=x^{2}|\tau|^{-1} p_{0}$, so $\sigma(\square)=x^{-2}|\tau|^{2} a_{N}$. As $x^{2} i\left[A_{l}, \square\right] \in \Psi_{\mathrm{e}}^{2}(M)$ and $A_{0}=\mathrm{Id}$, it suffices to prove that for suitable $c_{l j}$, homogeneous degree 1 with $\left.c_{l j}\right|_{\mathcal{F}}=0$,

$$
\sigma\left(x^{2} i\left[A_{l}, \square\right]\right)=\sum_{j=1}^{N} c_{l j}|\tau| a_{j} .
$$

But $\sigma\left(x^{2} i\left[A_{l}, \square\right]\right)=-x^{2} H_{p_{0}}\left(|\tau| a_{l}\right)=-H_{p_{0}}\left(|\tau| a_{l}\right)$ is homogeneous of degree 2 , vanishing at $\dot{\mathcal{F}}$, so (4.6) follows, though not necessarily with $\left.c_{l j}\right|_{\mathcal{F}}=0$. 
By Lemma 2.3 and the part of the statement of Lemma 4.4 regarding $d a_{l}(q), d a_{l}$ is an eigenvector of the linearization of $\sigma H_{p_{0}}$ with eigenvalue $-\hat{\xi}$, and $d \sigma$ is also an eigenvector with eigenvalue $-\hat{\xi}$. Correspondingly, using the homogeneity and working on ${ }^{\mathrm{e}} T^{*} M, \sigma H_{p_{0}}\left(\sigma^{-1} a_{l}\right)=\left(\sigma H_{p_{0}} \sigma^{-1}\right) a_{l}+$ $\sigma^{-1}\left(\sigma H_{p_{0}}\right)\left(a_{l}\right)$ vanishes at $\partial \dot{\mathcal{F}}$, so we may indeed take $\left.c_{l j}\right|_{\partial \dot{\mathcal{F}}}=0$.

The final piece of information we needed to analyze propagation of regularity with respect to $\mathcal{M}$ is to recall from Lemma 2.3 that $x$, resp. $\sigma$ are eigenvalues of the linearization of $\sigma H_{p}$ of eigenvalue $\hat{\xi}$, resp. $-\hat{\xi}$, and correspondingly for $W_{m, l} \in \Psi_{\mathrm{e}}^{m, l}(M)$ with symbol $w_{m, l}=|\tau|^{m} x^{l}=\sigma^{-m} x^{l}$,

$$
i\left[W_{m, l}, \square\right]=W_{m-1, l-1} C_{0}, \text { where } C_{0} \in \Psi_{\mathrm{e}}^{0,0}(M),\left.\sigma\left(C_{0}\right)\right|_{\partial \dot{\mathcal{F}}}=-(m+l) \hat{\xi} .
$$

\section{Domains}

An essential ingredient in our use of the edge calculus will be the identification of domains of powers of the Laplacian with weighted edge Sobolev spaces.

Definition 5.1. Let $\mathcal{D}$ denote the Friedrichs form domain of $\Delta$, i.e. the closure of $\dot{\mathcal{C}}^{\infty}(X)$ with respect to the norm $\left(\|d u\|_{L_{g}^{2}}^{2}+\|u\|_{L_{g}^{2}}^{2}\right)^{1 / 2}$. Let $\mathcal{D}_{s}$ denote the corresponding domain of $\Delta^{s / 2}$ (hence $\mathcal{D}=\mathcal{D}_{1}$ ), and $\mathcal{D}^{\prime}=\mathcal{D}_{-1}$.

Recall that $L_{g}^{2}(X)=x^{-(f+1) / 2} L_{b}^{2}(X)$, and that we use the b weight in our definition of edge Sobolev spaces.

We remark that multiplication by elements of $\mathcal{C}_{Y}^{\infty}(X)$ (the subspace of $\mathcal{C}^{\infty}(X)$ consisting of fiber constant functions at $\left.\partial X\right)$ preserves $\mathcal{D}$, as for

$$
\phi \in \mathcal{C}_{Y}^{\infty}(X), V \in x^{-1} \mathcal{V}_{\mathrm{e}}(X)
$$

we have

$$
V \phi u=\phi V u+[V, \phi] u,
$$

and

$$
[V, \phi] \in \mathcal{C}^{\infty}(X)
$$

is bounded on $L_{g}^{2}$. Thus, $\mathcal{D}$ can be characterized locally away from $\partial X$, plus locally in $Y$ near $\partial X$ (i.e. near $\partial X$ the domain does not have a local characterization, but it is local in the base $Y$, so the non-locality is in the fiber $Z$ ).

The basic lemma is the following.

Lemma 5.2. We have, for $v \in \dot{\mathcal{C}}^{\infty}(X), f>1$,

$$
\left\|x^{-1} v\right\|^{2}+\left\|x^{-1} D_{z} v\right\|^{2}+\left\|D_{x} v\right\|^{2}+\left\|D_{y} v\right\|^{2} \leq C\|v\|_{\mathcal{D}}^{2} .
$$

Remark 5.3. This is the only result we need for the diffractive theorem, which uses the b-calculus. If $f=1$, we use more carefully crafted ps.d.o's to make sure that the control of $\|v\|$, rather than $\left\|x^{-1} v\right\|$, suffices -indeed, $\left\|x^{-1} v\right\|$ is not controlled by $\|v\|_{\mathcal{D}}$ if $f=1$. A fortiori, in the extreme case $f=0$, we do not control $\left\|x^{-1} v\right\|$ by the domain norm. 
That a reverse inequality to (5.1) holds too is immediate from the definition of $\mathcal{D}$ and the form of the metric.

Proof. As $\|v\|_{\mathcal{D}}^{2}=\|d v\|_{L_{g}^{2}}^{2}+\|v\|_{L_{g}^{2}}^{2}$, the form of the metric $g$ implies

$$
\left\|x^{-1} D_{z} v\right\|^{2}+\left\|D_{x} v\right\|^{2}+\left\|D_{y} v\right\|^{2} \leq C\|v\|_{\mathcal{D}}^{2} .
$$

So it remains to show that $\left\|x^{-1} v\right\|^{2} \leq C\|v\|_{\mathcal{D}}^{2}$ for $v$ supported near $\partial X$, which in turn will follow from $\left\|x^{-1} v\right\|^{2} \leq C\left(\left\|D_{x} v\right\|^{2}+\|v\|^{2}\right)$. Treating $y, z$ as parameters, this in turn is an immediate consequence of the standard analogous one-dimensional result (with respect to the measure $x^{f} d x$ ).

As an immediate consequence, we deduce the following:

Proposition 5.4. Suppose that either $s \in[0,1)$, or $s=1$ and $f>1$. Then $\mathcal{D}_{s}=x^{-(f+1) / 2+s} H_{\mathrm{e}}^{s}(X)$.

Proof. For $s=1, f>1$, this follows immediately from Lemma 5.2 and the definition of the Friedrichs quadratic form domain $\mathcal{D}$ as the closure of $\dot{\mathcal{C}}^{\infty}(X)$ with respect to $\|\cdot\|_{\mathcal{D}}$. Indeed, the lemma gives $\|u\|_{x^{-(f+1) / 2+1} H_{\mathrm{e}}^{1}(X)} \leq C\|u\|_{\mathcal{D}}$ for $u \in \dot{\mathcal{C}}^{\infty}(X)$; the analogous reverse inequality is immediate, so the density of $\dot{\mathcal{C}}^{\infty}(X)$ in both spaces proves the proposition for $s=1$. As $\mathcal{D}_{\theta}, \theta \in[0,1]$, resp. $x^{\theta-(f+1) / 2} H_{\mathrm{e}}^{\theta}(X)$, are the complex interpolation spaces for $\left(L^{2}, \mathcal{D}\right)$, resp. $\left(L^{2}, x^{1-(f+1) / 2} H_{\mathrm{e}}^{1}(X)\right)$, the proposition follows for $s \in[0,1], f>1$.

A different way of identifying $\mathcal{D}$, which indeed works for arbitrary $f$, is to note that working in a local coordinate chart $O^{\prime}$ in $Y$ over which the fibration of $\partial X$ is trivial, and extending the fibration to a neighborhood of $\partial X$, one has an open set $O$ in $X$ which one can identify with $[0, \epsilon)_{x} \times O^{\prime} \times Z$. As $\mathcal{D}$ is a $\mathcal{C}_{Y}^{\infty}(X)$ module, we only need to characterize the elements of $\mathcal{D}$ supported in compact subsets of $O$ which have a product decomposition. But directly from the definition of $\mathcal{D}$, such functions are exactly the correspondingly supported elements of

$$
H^{1}\left(Y ; L^{2}\left([0, \epsilon)_{x} \times Z ; x^{f} d x d z\right)\right) \cap L^{2}(Y ; D([0, \epsilon) \times Z)),
$$

where $D$ stands for the Friedrichs form domain of any conic Laplacian on $[0, \epsilon) \times Z$. These domains $D$ are well-known, and are described in [19, Equation (3.11)]. In particular, for $f>1, D([0, \epsilon) \times Z)=x^{1-(f+1) / 2} H_{\mathrm{b}}^{1}([0, \epsilon) \times Z)$, providing an alternative method of characterization of the domain $\mathcal{D}$ for $f>1$.

Now, $x^{1-(f+1) / 2} H_{\mathrm{e}}^{1}(X) \subset \mathcal{D}$ always (with a continuous inclusion) as both are completions of $\dot{\mathcal{C}}^{\infty}(X)$, with the first norm being stronger than the second. Complex interpolation with $L^{2}$ (of which both are a subspace) gives the inclusion $x^{\theta-(f+1) / 2} H_{\mathrm{e}}^{\theta}(X) \subset \mathcal{D}_{\theta}$ for $\theta \in[0,1]$.

To see the reverse inclusion, we proceed as follows. In order to simplify the notation, we localize to a product neighborhood $O$ of $\partial X$, but do not denote this explicitly. Since complex interpolation of the spaces $L^{2}=\mathcal{D}_{0}$ and $\mathcal{D}=\mathcal{D}_{1}$ yields $\mathcal{D}_{\theta}$ for $0 \leq \theta \leq 1$, with an analogous statement for 
$D([0, \epsilon) \times Z)$, while complex interpolation of $L^{2}$ and $H^{1}$ yields $H^{\theta}$ for $0 \leq$ $\theta \leq 1$, we deduce that

$$
\mathcal{D}_{\theta} \subset H^{\theta}\left(Y ; L^{2}\left([0, \epsilon)_{x} \times Z\right)\right) \cap L^{2}\left(Y ; D_{\theta}([0, \epsilon) \times Z)\right),
$$

meaning elements of $\mathcal{D}_{\theta}$ multiplied by a cutoff supported in $O$ identically 1 near $\partial X$ are in the right hand side. For $\theta \in[0,1), D_{\theta}([0, \epsilon) \times Z)=$ $x^{\theta-(f+1) / 2} H_{\mathrm{b}}^{\theta}([0, \epsilon) \times Z)$, even if $f=1$, so we deduce that for $\theta \in[0,1)$,

$$
\mathcal{D}_{\theta} \subset H^{\theta}\left(Y ; L^{2}\left([0, \epsilon)_{x} \times Z\right)\right) \cap L^{2}\left(Y ; x^{\theta-(f+1) / 2} H_{\mathrm{b}}^{\theta}([0, \epsilon) \times Z)\right) .
$$

The right side is exactly $x^{\theta-(f+1) / 2} H_{\mathrm{e}}^{\theta}(O)$. In fact, this follows from

$$
\begin{aligned}
H^{1}\left(Y ; L^{2}\left([0, \epsilon)_{x} \times Z\right)\right), L^{2}\left(Y ; x^{1-(f+1) / 2} H_{\mathrm{b}}^{1}([0, \epsilon) \times Z)\right) \\
\text { and } x^{1-(f+1) / 2} H_{\mathrm{e}}^{1}(O)
\end{aligned}
$$

being the form domains of commuting self-adjoint operators $A_{1}, A_{2}$, resp. $A=A_{1}+A_{2}$, with $A_{j} \geq \mathrm{Id}$, as this implies the non-trivial (and relevant) direction, i.e. that

$H^{\theta}\left(Y ; L^{2}\left([0, \epsilon)_{x} \times Z\right)\right) \cap L^{2}\left(Y ; x^{\theta-(f+1) / 2} H_{\mathrm{b}}^{\theta}([0, \epsilon) \times Z)\right) \subset x^{\theta-(f+1) / 2} H_{\mathrm{e}}^{\theta}(O)$ by using $A^{(\theta-z) / 2}$ to produce the holomorphic family used in the definition of complex interpolation. For instance, one may take $A_{1}=\Delta_{Y}$ for some metric on $Y$, and $A_{2}=\Delta_{[0, \epsilon) \times Z}+c x^{-2}$ for a conic metric and $c>0$ sufficiently large.

Thus, the proposition follows for $s \in[0,1)$ and $f$ arbitrary (as well as $s=1, f>1)$.

We write $\tilde{\mathcal{D}}$, etc. for the analogous spaces on $M$ :

Definition 5.5. $\|u\|_{\tilde{\mathcal{D}}}^{2}=\left\|D_{t} u\right\|_{L^{2}(M)}^{2}+\left\|d_{X} u\right\|_{L^{2}(M)}^{2}+\|u\|_{L^{2}(M)}^{2}$. We also write $\tilde{\mathcal{D}}([a, b])$ for the space with the same norm on $[a, b] \times X$.

We will require the following result about the interaction of the test module $\mathcal{M}$ from Definition 4.2 with the norm on $\tilde{\mathcal{D}}$ :

Lemma 5.6. Let $A \in \mathcal{M}$. Let $A_{\tilde{\mathcal{D}}}^{*}$ denote the adjoint of $A$ respect to the norm on $\tilde{\mathcal{D}}$. Then

$$
A_{\tilde{\mathcal{D}}}^{*}=A^{*}+B+R
$$

with $B \in \Psi_{\mathrm{e}}^{0,0}(M)$ and $R$ a smoothing operator mapping $\tilde{\mathcal{D}}(M) \rightarrow \mathcal{C}^{\infty}(M)$.

Proof. Let $\tilde{\square}=D_{t}^{2}+\Delta+1$. If $u, v \in \dot{\mathcal{C}}^{\infty}(M)$ have compact support, we have

$$
\begin{aligned}
\langle A u, v\rangle_{\tilde{\mathcal{D}}} & =\langle\tilde{\square} A u, v\rangle \\
& =\left\langle u, A^{*} v\right\rangle_{\tilde{\mathcal{D}}}+\langle[\tilde{\square}, A] u, v\rangle .
\end{aligned}
$$

Using the usual elliptic parametrix construction, we may write

$$
[\tilde{\square}, A]=B^{*} \tilde{\square}+R^{\prime}
$$


where $B^{*} \in \Psi_{\mathrm{e}}^{0,0}(M)$ and $R^{\prime} \in \Psi_{\mathrm{e}}^{-\infty,-2}(M)$. By the Riesz Lemma, $\left\langle R^{\prime} u, v\right\rangle=$ $\langle R u, v\rangle_{\tilde{\mathcal{D}}}$ for some smoothing operator $R$, so we obtain the desired form of the adjoint.

The domain $\mathcal{D}$ localizes with respect to fiber-constant functions. Thus, we make the definition:

Definition 5.7. For $u \in \mathcal{C}^{-\infty}(X)$, we say that $u \in \mathcal{D}_{\text {loc }}$ if $\phi u \in \mathcal{D}$ for all fiber constant $\phi \in \mathcal{C}_{c}^{\infty}(X)$. Similarly, for $u \in \mathcal{C}^{-\infty}(X)$, we say that $u \in \mathcal{D}_{\text {loc }}^{\prime}$ if $\phi u \in \mathcal{D}^{\prime}$ for all fiber constant $\phi \in \mathcal{C}_{c}^{\infty}(X)$.

We define the localized domains on $M$ analogously.

Note that if $u \in \mathcal{D}$ then certainly $u \in \mathcal{D}_{\text {loc }}$.

\section{WAVEFront Sets $(f>1)$}

Throughout this section, we assume $f>1$. For the $f=1$ case, see Section $10 .^{3}$

By convention all ps.d.o's in this section have Schwartz kernels supported in a fixed compact set $\tilde{K} \times \tilde{K}$, and we define associated local norms by fixing a function $\tilde{\phi} \in \mathcal{C}_{c}^{\infty}(M)$ equal to 1 on neighborhood of $\tilde{K}$; we let

$$
\|u\|_{\bullet, \text { loc }}=\|\tilde{\phi} u\|_{\bullet}
$$

for various choices of Sobolev space.

Lemma 6.1. If $V \in x^{-1} \mathcal{V}_{\mathrm{e}}(M)$ and $A \in \Psi_{\mathrm{b} \infty}^{m}(M)$ then

$$
[A, V] \in x^{-1} \Psi_{\mathrm{b} \infty}^{m}(M) .
$$

(Recall that the calculus with conormal estimates, $\Psi_{\mathrm{b} \infty}^{*}(M)$, is described at the end of $\S 3$.)

Proof. The result follows from the fact that $V \in x^{-1} \operatorname{Diff}_{\mathrm{b}}^{1}(M)$.

Lemma 6.2. Any $A \in \Psi_{\mathrm{b} \infty}^{0}(M)$ with compact support defines a continuous linear map $A: \tilde{\mathcal{D}} \rightarrow \tilde{\mathcal{D}}$ with a norm bounded by a seminorm of $A$ in $\Psi_{\mathrm{b} \infty}^{0}(M)$.

Moreover, for any $K \subset M$ compact, any $A \in \Psi_{\mathrm{b} \infty}^{0}(M)$ with proper support defines a continuous map from the subspace of $\tilde{\mathcal{D}}$ consisting of distributions supported in $K$ to $\tilde{\mathcal{D}}_{c}$.

Proof. The norm on $\tilde{\mathcal{D}}$ is $\left(\left\|d_{M} u\right\|^{2}+\|u\|^{2}\right)^{1 / 2}$, and $\mathcal{C}_{c}^{\infty}\left(M^{\circ}\right)$ is dense on $\tilde{\mathcal{D}}$, so it suffices to show that for $u \in \mathcal{C}_{C}^{\infty}\left(M^{\circ}\right),\|A u\|_{L^{2}} \leq C\|u\|_{L^{2}}$, and $\|V A u\|_{L^{2}} \leq C\left(\left\|d_{M} u\right\|+\|u\|\right)$ for $V \in x^{-1} \mathcal{V}_{\mathrm{e}}(M)$. As $\Psi_{\mathrm{b} \infty}^{0}(M)$ is bounded on $L^{2}$, the first claim is clear. Moreover,

$$
\|V A u\|_{L^{2}} \leq\|A V u\|_{L^{2}}+\|[V, A] u\|_{L^{2}} \leq C\|V u\|_{L^{2}}+C\left\|x^{-1} u\right\|_{L^{2}}
$$

\footnotetext{
${ }^{3}$ As always, for the case of a manifold with boundary, i.e. $f=0$, we refer the reader to $[17,18]$.
} 
where we used the previous lemma for the second term, and also that $\Psi_{\mathrm{b} \infty}^{0}(M)$ is bounded on $L^{2}$. Finally, as $f>1,\left\|x^{-1} u\right\|_{L^{2}} \leq C\left(\left\|D_{x} u\right\|_{L^{2}}+\right.$ $\left.\|u\|_{L^{2}}\right)$, finishing the proof.

We note that this lemma is in fact false without the assumption $f>1$.

We are now able to follow the treatment of [25] almost verbatim, using the preceding Lemma in place of [25, Lemma 3.2].

Definition 6.3. For $m \geq 0$, we define $H_{\tilde{\mathcal{D}}, b, c}^{m}(M)$ as the subspace of $\tilde{\mathcal{D}}$ consisting of $u \in \tilde{\mathcal{D}}$ with $\operatorname{supp} u$ compact and $A u \in \tilde{\mathcal{D}}$ for some (hence any, see [25]) $A \in \Psi_{\mathrm{b}}^{m}(M)$ (with compact support) which is elliptic over $\operatorname{supp} u$, i.e. $A$ such that such that $\sigma_{\mathrm{b}, m}(A)(q) \neq 0$ for any $q \in{ }^{\mathrm{b}} T_{\operatorname{supp} u}^{*} M \backslash 0$.

We let $H_{\tilde{\mathcal{D}}, b \text {,loc }}^{m}(M)$ be the subspace of $\tilde{\mathcal{D}}_{\text {loc }}$ consisting of $u \in \tilde{\mathcal{D}}_{\text {loc }}$ such that for any $\phi \in \mathcal{C}_{c}^{\infty}(M), \phi u \in H_{\tilde{\mathcal{D}}, b, c}^{m}(M)$.

Thus, $H_{\tilde{\mathcal{D}}, b, \text { loc }}^{m}(M)$ consists of distributions conormal to finite order relative to $\tilde{\mathcal{D}}$. We also define the spaces with a negative order of conormal regularity relative to $\tilde{\mathcal{D}}$ :

Definition 6.4. Let $m<0$, and $A \in \Psi_{\mathrm{b}}^{-m}(M)$ be elliptic on ${ }^{\mathrm{b}} S^{*} M$ with proper support. We let $H_{\tilde{\mathcal{D}}, \mathrm{b}, c}^{m}(M)$ be the space of all $u \in \mathcal{C}^{-\infty}(M)$ of the form $u=u_{1}+A u_{2}$ with $u_{1}, u_{2} \in \tilde{\mathcal{D}}_{c}$. We let

$$
\|u\|_{H_{\tilde{\mathcal{D}}, \mathrm{b}, c}^{m}(M)}=\inf \left\{\left\|u_{1}\right\|_{\tilde{\mathcal{D}}}+\left\|u_{2}\right\|_{\tilde{\mathcal{D}}}: u=u_{1}+A u_{2}\right\} .
$$

We also let $H_{\tilde{\mathcal{D}} \text {,b,loc }}^{m}(M)$ be the space of all $u \in \mathcal{C}^{-\infty}(M)$ such that $\phi u \in$ $H_{\tilde{\mathcal{D}}, \mathrm{b}, c}^{m}(M)$ for all $\phi \in \mathcal{C}_{c}^{\infty}(M)$.

The spaces $H_{\tilde{\mathcal{D}}, \mathrm{b}, \mathrm{c}}^{m}$ and $H_{\tilde{\mathcal{D}} \text {,b,loc }}^{m}$ do not depend on the choice of $A$; see [25]. With this definition, $A \in \Psi_{\mathrm{b} \infty}^{k}(M)$ defines a map

$$
A: H_{\tilde{\mathcal{D}}, b, c}^{m}(M) \rightarrow H_{\tilde{\mathcal{D}}, b, c}^{m-k}(M) ;
$$

if the supports are kept in a fixed compact set, this is a continuous linear map between Banach spaces. We now proceed to microlocalize these spaces.

Definition 6.5. Let $u \in H_{\tilde{\mathcal{D}} \text {,b,loc }}^{s}$ for some $s$ and let $m \geq 0$. We say that $q \in{ }^{\mathrm{b}} T^{*} M \backslash 0$ is not in $\mathrm{WF}_{\mathrm{b}, \tilde{\mathcal{D}}}^{m}(u)$ if there exists $A \in \Psi_{\mathrm{b}}^{m}(M)$ such that $\sigma_{\mathrm{b}, m}(A)(q) \neq 0$ and $A u \in \tilde{\mathcal{D}}$.

For $m=\infty$, we say that $q \in{ }^{\mathrm{b}} T^{*} M \backslash 0$ is not in $\mathrm{WF}_{\mathrm{b}, \tilde{\mathcal{D}}}^{m}(u)$ if there exists $A \in \Psi_{\mathrm{b}}^{0}(M)$ such that $\sigma_{\mathrm{b}, 0}(A)(q) \neq 0$ and $L A u \in \tilde{\mathcal{D}}$ for all $L \in \operatorname{Diff}_{\mathrm{b}}(M)$, i.e. if $A u \in H_{\tilde{\mathcal{D}}, b, \text { loc }}^{\infty}(M)$.

We also use the notation $\mathrm{WF}_{\mathrm{b}, \tilde{\mathcal{D}}}(u)=\mathrm{WF}_{\mathrm{b}, \tilde{\mathcal{D}}}^{\infty}(u)$. 
Remark 6.6. Note that $\mathrm{WF}_{\mathrm{b}, \tilde{\mathcal{D}}}(u)$ is only defined for $u \in \bigcup_{s} H_{\tilde{\mathcal{D}} \text {,b,loc }}^{s}(M)$. It is reasonable thus to call $\bigcup_{s} H_{\tilde{\mathcal{D}} \text {,b,loc }}^{s}(M)$ the space of $\tilde{\mathcal{D}}$-admissible distributions, and to refer to solutions of $\square u=0$ with $u \in \bigcup_{s} H_{\tilde{\mathcal{D}}, \mathrm{b}, \text { loc }}^{s}(M)$ as admissible solutions - even, in a more general setting, if $\square$ is the 'Laplacian' of a general pseudo-Riemannian metric.

We recall the definition of the operator wave front set of a family of ps.d.o's.

Definition 6.7. Suppose that $\mathcal{B}$ is a bounded subset of $\Psi_{\mathrm{b} \infty}^{k}(M)$, and $q \in$ ${ }^{\mathrm{b}} S^{*} M$. We say that $q \notin \mathrm{WF}_{\mathrm{b}}^{\prime}(\mathcal{B})$ if there is some $A \in \Psi_{\mathrm{b}}(M)$ which is elliptic at $q$ such that $\{A B: B \in \mathcal{B}\}$ is a bounded subset of $\Psi_{\mathrm{b}}^{-\infty}(M)$.

Note that the wave front set of a family $\mathcal{B}$ is only defined for bounded families. It can be described directly in terms of quantization of (full) symbols, much like the operator wave front set of a single operator. All standard properties of the operator wave front set also hold for a family; e.g. if $E \in \Psi_{\mathrm{b}}(M)$ with $\mathrm{WF}_{\mathrm{b}}^{\prime}(E) \cap \mathrm{WF}_{\mathrm{b}}^{\prime}(\mathcal{B})=\emptyset$ then $\{B E: B \in \mathcal{B}\}$ is bounded in $\Psi_{\mathrm{b}}^{-\infty}(M)$.

The key lemma here concerns families $\mathcal{B}$ with wave front set disjoint from a wave front set $\mathrm{WF}_{\mathrm{b}, \tilde{\mathcal{D}}}^{k}(u)$ of a distribution $u$; it is the quantitative version of the microlocality of $\Psi_{\mathrm{b} \infty}(M)$ :

$$
\mathrm{WF}_{\mathrm{b}, \tilde{\mathcal{D}}}^{m-k}(B u) \subset \mathrm{WF}_{\mathrm{b}}^{\prime}(B) \cap \mathrm{WF}_{\mathrm{b}, \tilde{\mathcal{D}}}^{m}(u), B \in \Psi_{\mathrm{b} \infty}^{k}(M),
$$

see [25, Lemma 3.9].

Lemma 6.8. Suppose that $K \subset{ }^{\mathrm{b}} S^{*} M$ is compact, and $U$ a neighborhood of $K$ in ${ }^{\mathrm{b}} S^{*} M$. Let $Q \in \Psi_{\mathrm{b}}^{k}(M)$ be elliptic on $K$ with $\mathrm{WF}_{\mathrm{b}}^{\prime}(Q) \subset U$. Let $\mathcal{B}$ be a bounded subset of $\Psi_{\mathrm{b} \infty}^{k}(M)$ with $\mathrm{WF}_{\mathrm{b}}^{\prime}(\mathcal{B}) \subset K$. Then for any $s \in \mathbb{R}$ there is a constant $C>0$ such that

$$
\|B u\|_{\tilde{\mathcal{D}}} \leq C\left(\|u\|_{H_{\tilde{\mathcal{D}}, \mathrm{b}, l o c}^{s}}+\|Q u\|_{\tilde{\mathcal{D}}}\right) .
$$

for $B \in \mathcal{B}, u \in H_{\tilde{\mathcal{D}}, \mathrm{b}, \text { loc }}^{s}$ with $\mathrm{WF}_{\mathrm{b}, \tilde{\mathcal{D}}}^{k}(u) \cap U=\emptyset$,

For the proof, see [25, Lemma 3.13 and Lemma 3.18].

Remark 6.9. Instead of working with $\tilde{\mathcal{D}}$, one can also work with $\tilde{\mathcal{D}}^{\prime}$, as $\Psi_{\mathrm{b} \infty}^{0}(M)$ acts on it by duality. All the above results have their analogues with $\mathrm{WF}_{\mathrm{b}, \tilde{\mathcal{D}}}$ replaced by $\mathrm{WF}_{\mathrm{b}, \tilde{\mathcal{D}}^{\prime}}$.

We will also employ a wave front set on a quotient space of ${ }^{\mathrm{b}} S^{*} M$. In $\S 7$ we will define a space ${ }^{\mathrm{b}} \dot{S}^{*} M$ with a proper, surjective map $p:{ }^{\mathrm{b}} S^{*} M \rightarrow{ }^{\mathrm{b}} \dot{S}^{*} M$; ${ }^{\mathrm{b}} \dot{S}^{*} M$ will be equipped with the quotient topology. In this case, by abusing the notation slightly, we use the following terminology:

Definition 6.10. $\mathrm{WF}_{\mathrm{b}, \tilde{\mathcal{D}}}(u)$ and $\mathrm{WF}_{\mathrm{b}, \tilde{\mathcal{D}}^{\prime}}(u)$, considered as subsets of ${ }^{\mathrm{b}} \dot{S}^{*} M$, are the images under the quotient map $p$ of $\mathrm{WF}_{\mathrm{b}, \tilde{\mathcal{D}}}(u)$ and $\mathrm{WF}_{\mathrm{b}, \tilde{\mathcal{D}}^{\prime}}(u)$, considered as subsets of ${ }^{\mathrm{b}} S^{*} M$. 
Thus, for $q \in{ }^{\mathrm{b}} \dot{S}^{*} M, q \notin \mathrm{WF}_{\mathrm{b}, \tilde{\mathcal{D}}}(u)$ if and only if $p^{-1}(q) \cap \mathrm{WF}_{\mathrm{b}, \tilde{\mathcal{D}}}(u)=\emptyset$, i.e. $u$ is well-behaved at all pre-images of $q$.

\section{EDGE/B RELATIONSHIP}

Let $N$ denote an edge manifold with fibration

$$
Z \rightarrow \partial N \stackrel{\pi_{0}}{\rightarrow} W .
$$

Canonical coordinates on ${ }^{\mathrm{e}} T^{*} N$ induced by coordinates $(x, w, z)$ (with $w$ pull-backs of coordinates on $W$ ) on $N$ are $(x, w, z, \xi, \mu, \zeta)$ corresponding to writing covectors in ${ }^{\mathrm{e}} T^{*} N$ as

$$
\xi \frac{d x}{x}+\mu \cdot \frac{d w}{x}+\zeta \cdot d z
$$

Analogously, canonical coordinates on ${ }^{\mathrm{b}} T^{*} N$ are $(x, w, z, \underline{\xi}, \underline{\mu}, \underline{\zeta})$ corresponding to writing covectors in ${ }^{\mathrm{b}} T^{*} N$ as

$$
\underline{\xi} \frac{d x}{x}+\underline{\mu} \cdot d w+\underline{\zeta} \cdot d z .
$$

Let $\pi$ denote the bundle map ${ }^{\mathrm{e}} T^{*} N \rightarrow{ }^{\mathrm{b}} T^{*} N$ given in canonical coordinates by

$$
\pi(x, w, z, \xi, \mu, \zeta)=(x, w, z, x \xi, \mu, x \zeta) .
$$

This map can be obtained more invariantly by identifying ${ }^{\mathrm{e}} T^{*} N$ with the bundle ${ }^{x \mathrm{e}} T^{*} N:=x^{\mathrm{e}} T^{*} N$, i.e. the bundle whose sections can be written as $x$ times an edge one-form; the identification is via multiplication by $x$, naturally. The map $\pi$ is then the inclusion map of this bundle into ${ }^{\mathrm{b}} T^{*} M$. We define the compressed cotangent bundle by setting

$$
\begin{aligned}
{ }^{\mathrm{b}} \dot{T}^{*} N & =\pi\left({ }^{\mathrm{e}} T^{*} N\right) / Z, \\
\dot{\pi}:{ }^{\mathrm{e}} T^{*} N & \rightarrow{ }^{\mathrm{b}} \dot{T}^{*} N
\end{aligned}
$$

the projection, where, here and henceforth, we take the quotient by $Z$ to act only over the boundary (where it is defined) and the topology to be given by the quotient topology. We remark that ${ }^{\mathrm{b}} \dot{T}^{*}{ }_{\partial N} N$ can be identified with $T^{*} W$.

In our geometric setting, we have $N=M:=\mathbb{R} \times X, W=\mathbb{R} \times Y$, and the $w$ variables above are replaced by $w=(t, y)$, and correspondingly $\mu=(\tau, \eta)$, hence $\underline{\mu}=(\underline{\tau}, \underline{\eta})$ and the canonical one-form on the b-cotangent bundle is

$$
\underline{\xi} \frac{d x}{x}+\underline{\tau} d t+\underline{\eta} \cdot d y+\underline{\zeta} \cdot d z
$$

Below it is much more convenient to work with the cosphere bundles rather than the cotangent bundles. These are defined as

$$
{ }^{\mathrm{b}} S^{*} M=\left({ }^{\mathrm{b}} T^{*} M \backslash 0\right) / \mathbb{R}^{+},{ }^{\mathrm{b}} \dot{S}^{*} M={ }^{\mathrm{b}} S^{*} M / Z,{ }^{\mathrm{e}} S^{*} M=\left({ }^{\mathrm{e}} T^{*} M \backslash 0\right) / \mathbb{R}^{+},
$$

where 0 denotes the zero sections of the respective bundles and $\mathbb{R}^{+}$acts by scalar multiplication in the fibers. The maps $\pi$ and $\dot{\pi}$ commute with 
the $\mathbb{R}^{+}$-action on the cotangent bundles, but they do map some non-zero covectors to the zero section of ${ }^{\mathrm{b}} T^{*} M$ (at $x=0$ ), so they do not descend to maps ${ }^{\mathrm{e}} S^{*} M \rightarrow{ }^{\mathrm{b}} \dot{S}^{*} M$. (We remark that ${ }^{\mathrm{e}} S^{*} M$ and ${ }^{x}{ }^{\mathrm{e}} S^{*} M$ are naturally identified, which is one of the reasons for working with cosphere bundles.) Nonetheless, we introduce the notation

$$
\begin{aligned}
& \pi\left({ }^{\mathrm{e}} S^{*} M\right)=\left(\pi\left({ }^{\mathrm{e}} T^{*} M\right) \backslash 0\right) / \mathbb{R}^{+} \subset{ }^{\mathrm{b}} S^{*} M, \\
& \dot{\pi}\left({ }^{\mathrm{e}} S^{*} M\right)=\left(\dot{\pi}\left({ }^{\mathrm{e}} T^{*} M\right) \backslash 0\right) / \mathbb{R}^{+} \subset{ }^{\mathrm{b}} \dot{S}^{*} M
\end{aligned}
$$

for convenience.

However, letting $p=\sigma_{e}\left(x^{2} \square\right) \in \mathcal{C}^{\infty}\left({ }^{\mathrm{e}} T^{*} M \backslash 0\right)$, we have

$$
\left.p\right|_{x=0}=\tau^{2}-\left(\xi^{2}+|\eta|_{h}^{2}+|\zeta|_{k}^{2}\right) .
$$

Since

$$
\pi(0, t, y, z, \xi, \tau, \eta, \zeta)=(0, w, z, 0, \tau, \eta, 0)
$$

on the characteristic set $p^{-1}(\{0\}) \subset{ }^{\mathrm{e}} T^{*} M$ of $\square$, where $\tau \neq 0$, non-zero covectors are mapped to non-zero covectors by $\pi$, hence also by $\dot{\pi}$. (In other words, $\partial M$ is non-characteristic.) Thus $\pi, \dot{\pi}$ also define maps, denoted with the same letter:

$$
\pi: \Sigma \rightarrow{ }^{\mathrm{b}} S^{*} M, \dot{\pi}: \Sigma \rightarrow{ }^{\mathrm{b}} \dot{S}^{*} M, \Sigma=p^{-1}(\{0\}) / \mathbb{R}^{+} .
$$

We set

$$
\dot{\Sigma}=\dot{\pi}(\Sigma)
$$

this is the compressed characteristic set.

In order to obtain coordinates on a subset of the cosphere bundle from the canonical coordinates on a cotangent bundle we need to fix a homogeneous degree 1 function that does not vanish on the cone corresponding to this subset. In our case, on $\Sigma,|\tau|$ can be taken as a canonical choice. We thus let

$$
\hat{\xi}=\frac{\xi}{|\tau|}, \hat{\eta}=\frac{\eta}{|\tau|}, \hat{\zeta}=\frac{\zeta}{|\tau|}
$$

(so $\hat{\tau} \equiv 1$ ), and likewise, in the 'b-coordinates' of (7.1),

$$
\underline{\hat{\xi}}=\frac{\underline{\xi}}{|\underline{\tau}|}, \underline{\hat{\eta}}=\frac{\underline{\eta}}{|\underline{\tau}|}, \hat{\hat{\zeta}}=\frac{\underline{\zeta}}{|\underline{\tau}|} .
$$

In these coordinates, at $x=0, \Sigma$ is given by $\hat{\xi}^{2}+|\hat{\eta}|_{h}^{2}+|\hat{\zeta}|_{k}^{2}=1$, while at $x=0, \dot{\Sigma}$ is given by $\underline{\hat{\xi}}=0, \underline{\hat{\zeta}}=0,|\underline{\hat{\eta}}|_{h}^{2} \leq 1$ (with the subscripts $h, k$ as usual denoting lengths with respect to the indicated metrics).

Lemma 7.1. Every neighborhood of a point $q_{0}=\left(t_{0}, y_{0}, \tau=1, \underline{\hat{\eta}}_{0}\right) \in \dot{\Sigma}$ in $\dot{\Sigma}$ contains an open set of the form

$$
\left\{q:|x(q)|^{2}+\left|y(q)-y_{0}\right|^{2}+\left|t(q)-t_{0}\right|^{2}+\left|\underline{\hat{\eta}}(q)-\underline{\hat{\eta}}_{0}\right|^{2}<\delta\right\} .
$$


Proof. Let $\rho:{ }^{\mathrm{b}} S^{*} M \rightarrow{ }^{\mathrm{b}} \dot{S}^{*} M$ be the quotient map. A neighborhood $U$ of $q_{0}$ in $\dot{\Sigma}$ is, by definition, of the form $U^{\prime} \cap \dot{\Sigma}$ where $U^{\prime} \subset{ }^{\mathrm{b}} \dot{S}^{*} M$ is open. But, as $U^{\prime}$ being open, $Z$ is compact, and $q_{0} \in U^{\prime}, U^{\prime}$ contains an open set of the form

$$
\begin{aligned}
\left\{q \in{ }^{\mathrm{b}} \dot{S}^{*} M:|x(q)|^{2}\right. & +\left|y(q)-y_{0}\right|^{2}+\left|t(q)-t_{0}\right|^{2} \\
& \left.+|\underline{\hat{\xi}}(q)|^{2}+\left|\underline{\hat{\gamma}}(q)-\underline{\hat{\eta}}_{0}\right|^{2}+|\underline{\hat{\zeta}}(q)|^{2}<\delta^{\prime}\right\}
\end{aligned}
$$

for some $\delta^{\prime}>0$. But $\dot{\Sigma}=\pi(\Sigma)$, so on $\dot{\Sigma}, \underline{\hat{\xi}}(q)=x\left(q^{\prime}\right) \hat{\xi}\left(q^{\prime}\right)$ with $q^{\prime} \in \Sigma$, $q=\dot{\pi}\left(q^{\prime}\right)$, so $|\hat{\xi}(q)| \leq x(q)\left|\hat{\xi}\left(q^{\prime}\right)\right| \leq x(q)$, hence on a set in $\dot{\Sigma}$ with $x(q)^{2}<\delta^{\prime \prime}$, we have in fact $|\hat{\xi}(q)|^{2}<\delta^{\prime \prime}$, and similarly, $|\hat{\zeta}(q)|^{2}<C \delta^{\prime \prime}$. Thus, the set

$$
\left\{q \in \dot{\Sigma}:|x(q)|^{2}+\left|y(q)-y_{0}\right|^{2}+\left|t(q)-t_{0}\right|^{2}+\left|\underline{\hat{\eta}}(q)-\underline{\hat{\eta}}_{0}\right|^{2}<(C+2)^{-1} \delta^{\prime}\right\}
$$

is indeed contained in (7.3), proving the lemma.

Lemma 7.2. $\dot{\Sigma}$ is a metrizable space.

Proof. This follows from ${ }^{\mathrm{b}} \dot{S}^{*} M$ being metrizable, which can be shown easily directly. We provide an alternative argument From Lemma 7.1 it is immediate that $\dot{\Sigma}$ is regular, i.e. if $q_{0} \in \dot{\Sigma}, F \subset \dot{\Sigma}$ closed, $q_{0} \notin F$, then there are disjoint neighborhoods of $U$ of $q_{0}$ and $U^{\prime}$ of $F$ : indeed, we can use sub- and superlevel sets of the continuous function $f_{q_{0}}(q)=|x(q)|^{2}+$ $\left|y(q)-y_{0}\right|^{2}+\left|t(q)-t_{0}\right|^{2}+\left|\underline{\hat{\eta}}(q)-\underline{\hat{\eta}}_{0}\right|^{2}$. If now $K$ is compact, $F$ is closed, for each $q_{0} \in K$ there is a function $\chi_{q_{0}} \in \mathcal{C}_{c}^{\infty}(\mathbb{R})$, identically 1 near 0 such that $\operatorname{supp}\left(\chi_{q_{0}} \circ f_{q_{0}}\right) \cap F=\emptyset$. Let $V_{q_{0}}=\left\{q: \chi_{q_{0}} \circ f_{q_{0}}>1 / 2\right\}$, then $\left\{V_{q_{0}}: q_{0} \in K\right\}$ is an open cover of $K$. Let $\left\{V_{q_{1}}, \ldots, V_{q_{k}}\right\}$ be a finite subcover. Then $F(q)=\sum_{j=1}^{k}\left(\chi_{q_{j}} \circ f_{q_{j}}\right)(q)$ satisfies $F>1 / 2$ on $K, F=0$ on $F$, so again sub- and superlevel sets of $F$ can be used as disjoint neighborhoods of $F$ and $K$. If $K$ is closed but not compact, the sum can still be made locally finite, proving that $\dot{\Sigma}$ is normal. This proves the lemma.

We may define the following three subsets of $\pi\left({ }^{\mathrm{e}} S_{\partial M}^{*} M\right)$ ('elliptic,' 'glancing' and 'hyperbolic'):

$$
\begin{aligned}
& \mathcal{E}=\pi\left({ }^{\mathrm{e}} S_{\partial M}^{*} M\right) \backslash \pi(\Sigma), \\
& \mathcal{G}=\left\{q \in \pi\left({ }^{\mathrm{e}} S_{\partial M}^{*} M\right): \operatorname{Card}\left(\pi^{-1}(q) \cap \Sigma\right)=1\right\}, \\
& \mathcal{H}=\left\{q \in \pi\left({ }^{\mathrm{e}} S_{\partial M}^{*} M\right): \operatorname{Card}\left(\pi^{-1}(q) \cap \Sigma\right) \geq 2\right\} .
\end{aligned}
$$

In coordinates, we have

$$
\pi(0, t, y, z, \xi, \hat{\tau}, \hat{\eta}, \zeta)=(0, t, y, z, 0, \hat{\tau}, \hat{\eta}, 0)
$$

hence the three sets are defined by $\left\{\underline{\hat{\tau}}^{2}<|\underline{\hat{\eta}}|^{2}\right\},\left\{\underline{\hat{\tau}}^{2}=|\underline{\hat{\eta}}|^{2}\right\},\left\{\underline{\hat{\tau}}^{2}>|\underline{\hat{\eta}}|^{2}\right\}$ respectively inside $\pi\left({ }^{\mathrm{e}} S_{\partial M}^{*} M\right)$, which is given by $x=0, \underline{\hat{\zeta}}=0, \underline{\hat{\xi}}=0$. 
We may also define the corresponding sets in ${ }^{\mathrm{b}} \dot{S}^{*}{ }_{\partial M} M$ (hence quotiented by $Z$ and denoted with a dot):

$$
\begin{aligned}
& \dot{\mathcal{E}}=\mathcal{E} / Z, \\
& \dot{\mathcal{G}}=\mathcal{G} / Z, \\
& \dot{\mathcal{H}}=\mathcal{H} / Z
\end{aligned}
$$

Remark 7.3. The hyperbolic and glancing sets can be visualized as (co-) vectors at the boundary that are respectively transverse to the geometric boundary $Y$ and tangent to it. A major difference from the situation of manifolds with boundary is that given a point in $\partial X$ and tangential momenta $\underline{\hat{\tau}}, \underline{\hat{\eta}}$ with $\hat{\underline{\tau}}^{2}>|\underline{\hat{\eta}}|^{2}$ we have specified not just a single point in the hyperbolic set but a whole manifold of them parametrized by $Z$. If $X=\left[\mathbb{R}^{3} ; \gamma\right]$ is given by the blowup of a smooth curve $\gamma$, then we may visualize these points as the tangents to the cone of rays emanating from a single point in $\gamma$ making a fixed angle to $\gamma$ (hence striking the blown-up space at all possible points in a fixed fiber). It is thus the whole cone that is being considered at once when we specify a point in the quotient space $\dot{\mathcal{H}}$.

Remark 7.4. In the related (but distinct) case of manifolds with corners with smooth nondegenerate metrics, one defines the hyperbolic, glancing, and elliptic sets with respect to the most singular boundary stratum (smallest corner) at a given point - cf. $[12,25]$. Upon blowup of this stratum, we recover the immediate analogs of the definitions above. For certain more refined results, it is necessary to keep track of the relationships of rays with the different boundary hypersurfaces; for instance, a ray might be hyperbolic with respect to one hypersurface and glancing with respect to another.

Bicharacteristics are usually defined as curves in the cotangent bundle. It is in fact more natural to define them in the cosphere bundle. However, the Hamilton vector field of a homogeneous degree $m$ function is homogenous of degree $m-1$, so the curves in the cosphere bundle are only defined up to reparametrization unless $m=1$. To fix a parameterization, we renormalize $\sigma_{e}(\square)$ to make it homogeneous of degree 1 , by considering

$$
\hat{p}=x|\tau|^{-1} \sigma_{e}(\square) \in x^{-1} \mathcal{C}^{\infty}\left({ }^{\mathrm{e}} T^{*} M \backslash 0\right) .
$$

Note that $\hat{p}$ is actually independent of the choice of $x ; x^{-1} \tau=\sigma_{e}\left(D_{t}\right)$, which is constant along bicharacteristics in $T^{*} M^{\circ}$, so the bicharacteristics do not 'slow down' as they approach $\partial M$, unlike the integral curves of $x^{2} H_{p}$. In particular, their projection to $X$ are constant speed geodesics. Then $H_{\hat{p}}$ is homogeneous of degree 0 , hence defines a vector field on ${ }^{\mathrm{e}} S_{M^{\circ}}{ }^{\circ} M$. Equivalently, on $\Sigma$, we can renormalize $H_{\sigma_{e}(\square)}$ :

$$
H_{\hat{p}}=|\tau|^{-1} x H_{\sigma_{e}(\square)}=-\frac{2}{|\tau| x} H, H=-2^{-1} x^{2} H_{\sigma_{e}(\square)},
$$


$H$ as in (2.2). Explicitly,

$$
\begin{aligned}
& -\frac{1}{2} H_{\hat{p}}= \\
& -\hat{\tau} \partial_{t}+\hat{\xi} \partial_{x}+x^{-1}\left(|\hat{\zeta}|^{2}+O\left(x^{2}\right) \hat{\zeta}^{2}+O\left(x^{2}\right) \hat{\eta} \hat{\zeta}+O(x) \hat{\eta}^{2}\right) \partial_{\hat{\xi}} \\
& +\left(x^{-1} \hat{\zeta}_{i} \bar{K}^{i j}+O(1) \hat{\eta}\right) \partial_{z_{j}}+\left(-\frac{1}{2} x^{-1} \hat{\zeta}_{i} \hat{\zeta}_{j} \frac{\partial \bar{K}^{i j}}{\partial z_{k}}+O(1) \hat{\eta} \hat{\zeta}+O(x) \hat{\eta}^{2}\right) \partial_{\hat{\zeta}_{k}} \\
& +\left(\hat{\eta}_{j} H^{i j}+O\left(x^{2}\right) \hat{\eta}+O(x) \hat{\zeta}\right) \partial_{y_{i}}+\left(O(1) \hat{\eta}^{2}+O(x) \hat{\eta} \hat{\zeta}+O(1) \hat{\zeta}^{2}\right) \partial_{\hat{\eta}_{i}} .
\end{aligned}
$$

While $H_{\hat{p}}$ is not a $\mathcal{C}^{\infty}$ vector field (it is $x H_{\hat{p}}$ that is $\mathcal{C}^{\infty}$ ) on ${ }^{\mathrm{e}} S^{*} M$, it acts on $\mathcal{C}^{\infty}$ functions independent of $z, \hat{\xi}, \hat{\zeta}$ at $x=0$, hence on pull-backs of functions from ${ }^{\mathrm{b}} \dot{S}^{*} M$.

Note that by definition

$$
\dot{\Sigma} \cap{ }^{b} T_{\partial M}^{*} M=\dot{\mathcal{G}} \cup \dot{\mathcal{H}} .
$$

Definition 7.5. Let $I$ be an interval. We say that a continuous map $\gamma: I \rightarrow \dot{\Sigma}$ is a generalized broken bicharacteristic if for all $f \in \mathcal{C}\left({ }^{\mathrm{b}} \dot{S}^{*} M\right)$ real valued with $\dot{\pi}^{*} f \in \mathcal{C}^{\infty}\left({ }^{\mathrm{e}} S^{*} M\right)$,

$$
\begin{aligned}
& \liminf _{s \rightarrow s_{0}} \frac{(f \circ \gamma)(s)-(f \circ \gamma)\left(s_{0}\right)}{s-s_{0}} \\
& \geq \inf \left\{H_{\hat{p}}\left(\dot{\pi}^{*} f\right)(q): q \in \dot{\pi}^{-1}\left(\gamma\left(s_{0}\right)\right) \cap \Sigma\right\}
\end{aligned}
$$

holds.

Remark 7.6. This definition may appear slightly complicated, especially as compared to the more explicit statement of the next lemma, which could be used as an alternative definition. However, it is the natural definition, for certainly any putative notion of generalized broken bicharacteristic should have the stated property. While it may be possible to strengthen the definition so as to rule out certain tangential rays in some cases (as happens in the smooth boundary setting), this would be a delicate matter. In $N$ body scattering one can also define generalized broken bicharacteristics as in Definition 7.5, but these do not have a simple characterization analogous to that given below.

Since ${ }^{\mathrm{e}} S^{*} M \ni q \mapsto H_{\hat{p}}\left(\dot{\pi}^{*} f\right)(q)$ is continuous for $f$ as in the definition, and as $\dot{\pi}^{-1}\left(\gamma\left(s_{0}\right)\right) \cap \Sigma$ is compact, the infimum on the right hand side is finite. Applying the same estimate to $-f$, we deduce that $f \circ \gamma$ is locally Lipschitz.

Lemma 7.7. Suppose $\gamma$ is a generalized broken bicharacteristic.

(1) If $\gamma\left(s_{0}\right) \in \dot{\mathcal{G}}$ then for all $f \in \mathcal{C}\left({ }^{\mathrm{b}} \dot{S}^{*} M\right)$ real valued with $\dot{\pi}^{*} f \in$ $\mathcal{C}^{\infty}\left({ }^{\mathrm{e}} S^{*} M\right), f \circ \gamma$ is differentiable at $s_{0}$ with

$$
(f \circ \gamma)^{\prime}\left(s_{0}\right)=H_{p}\left(\dot{\pi}^{*} f\right)(q),
$$


where $q$ is the unique point in $\dot{\pi}^{-1}\left(\gamma\left(s_{0}\right)\right) \cap \Sigma$.

(2) If $\gamma\left(s_{0}\right) \in \dot{\mathcal{H}}$ then there exists $\epsilon>0$ such that $\left|s-s_{0}\right|<\epsilon, s \neq s_{0}$ implies $x(\gamma(s)) \neq 0$, i.e. $\gamma(s)$ does not lie over the boundary.

Proof. Part (1) follows from the definition by applying it to both $f$ and $-f$, and noting that the set over which the inf is taken has a single element, so in fact $\lim _{s \rightarrow s_{0}}\left(s-s_{0}\right)^{-1}\left((f \circ \gamma)(s)-(f \circ \gamma)\left(s_{0}\right)\right)$ exists.

For part (2), consider $f=\hat{\xi} \in \mathcal{C}\left({ }^{\mathrm{b}} \dot{S}^{*} M\right)$, so $\dot{\pi}^{*} f \in \mathcal{C}^{\infty}\left({ }^{\mathrm{e}} S^{*} M\right)$, and indeed $\dot{\pi}^{*} f=x \hat{\xi}$. For $q \in \Sigma$ with $x(\bar{q})=0,(7.4)$ gives

$$
H_{\hat{p}} \dot{\pi}^{*} f(q)=\hat{\xi}^{2}(q)+|\hat{\zeta}|_{k}^{2}(q)=\hat{\tau}^{2}(q)-|\hat{\eta}|_{h}^{2}(q)>0,
$$

so $\dot{\pi}^{*} f$ is strictly increasing, and consequently non-zero in a punctured neighborhood of $s_{0}$, so the same holds for $f$. But on $\dot{\Sigma}$, at $x=0, f=0$, so we deduce that $x \neq 0$ in a punctured neighborhood of $s_{0}$ as claimed.

As an immediate corollary, we deduce that near points in $\dot{\mathcal{H}}$, generalized broken bicharacteristics consist of two bicharacteristic segments of $x^{2} H_{p}$ in ${ }^{\mathrm{e}} S^{*} M$ (projected to ${ }^{\mathrm{b}} \dot{S}^{*} M$ ), one incoming and one outgoing.

The following are useful general facts about generalized broken bicharacteristics.

Corollary 7.8. (cf. Lebeau, [12, Corollaire 2]) Suppose that $K$ is a compact subset of $\dot{\Sigma}$. Then there is a constant $C>0$ such that for all generalized broken bicharacteristics $\gamma: I \rightarrow K$, and for all functions $f$ on a neighborhood of $K$ in $\dot{\Sigma}$ with $\dot{\pi}^{*} f \in \mathcal{C}^{\infty}\left({ }^{\mathrm{e}} S^{*} M\right)$, one has the uniform Lipschitz estimate

$$
\left|f \circ \gamma\left(s_{1}\right)-f \circ \gamma\left(s_{2}\right)\right| \leq C\left\|\dot{\pi}^{*} f\right\|_{C^{2}}\left|s_{1}-s_{2}\right|, s_{1}, s_{2} \in I .
$$

In particular, (locally) the functions $x, y, t, \underline{\tau}$ and $\eta$ are Lipschitz on generalized broken bicharacteristics.

We also need to analyze the uniform behavior of generalized broken bicharacteristics. Here we quote Lebeau's results.

Proposition 7.9. (cf. Lebeau, [12, Proposition 5]) Suppose that $K$ is a compact subset of $\dot{\Sigma}, \gamma_{n}:[a, b] \rightarrow K$ is a sequence of generalized broken bicharacteristics which converge uniformly to $\gamma$. Then $\gamma$ is a generalized broken bicharacteristic.

Proposition 7.10. (cf. Lebeau, [12, Proposition 6]) Suppose that $K$ is a compact subset of $\dot{\Sigma},[a, b] \subset \mathbb{R}$ and

$$
\mathcal{R}=\{\text { generalized broken bicharacteristics } \gamma:[a, b] \rightarrow K\} .
$$

If $\mathcal{R}$ is not empty then it is compact in the topology of uniform convergence.

Proof. $\mathcal{R}$ is equicontinuous, as in Lebeau's proof, so the proposition follows from the theorem of Ascoli-Arzelà and Proposition 7.9.

Corollary 7.11. (Lebeau, [12, Corollaire 7]) If $\gamma:(a, b) \rightarrow \dot{\Sigma}$ is a generalized broken bicharacteristic then $\gamma$ extends to $[a, b]$. 
We now make the connection with the $\mathcal{F}, \dot{\mathcal{F}}$ notation introduced above. Given $q \in \mathcal{H}$, we recall from $\S 2$ that there exist unique maximally extended incoming/outgoing bicharacteristics $\gamma_{I / O}$, where $\operatorname{sgn} \xi= \pm \operatorname{sgn} \tau$, such that $q=\partial\left(\overline{\gamma_{\bullet}}\right) ;$ as above, we denote these curves

$$
\mathcal{F}_{\bullet, q} \cdot
$$

Likewise, for $p \in \dot{\mathcal{H}}$ we let

$$
\dot{\mathcal{F}}_{\bullet, p}=\bigcup_{q \in \pi_{0}^{-1} p} \mathcal{F}_{\bullet}, q .
$$

We will abuse notation slightly to write

$$
\dot{\mathcal{H}}_{I / O}=\partial \dot{\mathcal{F}}_{I / O}
$$

for the endpoints of incoming/outgoing hyperbfolic bicharacteristics at the boundary.

As opposed to generalized broken bicharacteristics, we may define a different flow relation, at least away from glancing rays, as follows:

Definition 7.12. Let $q, q^{\prime} \in \mathcal{H}$, with $\pi_{0}(q)=\pi_{0}\left(q^{\prime}\right)$. We say that

$$
\mathcal{F}_{I, q}, \mathcal{F}_{O, q^{\prime}}
$$

are related under the forward geometric flow (and vice-versa under the backward flow) if there exists a geodesic of length $\pi$ in $Z_{y(q)}$ connecting $z(q)$ and $z\left(q^{\prime}\right)$. If $a \in \mathcal{F}_{I, q}$ with $q \in \mathcal{H}$, we let the forward flowout of $a$ be the union of the forward geodesic segment through $a$ and all the $\mathcal{F}_{O, q^{\prime}}$ that are related to $\mathcal{F}_{I, q}$ under the forward geometric flow (and vice-versa for backward flow). (If the forward flow through $a$ stays in $M^{\circ}$, we simply let its forward flowout be the ordinary flowout under geodesic flow.)

We note that the flow relation generated by generalized broken bicharacteristics differs from that of Definition 7.12 as follows: a ray in $\mathcal{F}_{I, q}$ can be continued as a generalized broken bicharacteristic by any $\mathcal{F}_{O, q^{\prime}}$ with $\pi_{0}\left(q^{\prime}\right)=\pi_{0}(q)$; there is no requirement on the relative locations of $q, q^{\prime}$ in the fiber.

\section{ElLipticity}

First note that products of elements of $x^{-1} \operatorname{Diff}_{\mathrm{e}}^{1}(M)$ and $\Psi_{\mathrm{b}}^{m}(M)$ can be written with the products taken in either order:

Lemma 8.1. For any $Q \in x^{-1} \operatorname{Diff}_{\mathrm{e}}^{1}(M)$ and $A \in \Psi_{\mathrm{b}}^{m}(X)$ there exist $A^{\prime} \in$ $\Psi_{\mathrm{b}}^{m}(X), Q^{\prime} \in x^{-1} \operatorname{Diff}_{\mathrm{e}}^{1}(M)$ such that

$$
Q A=A Q+A^{\prime} Q^{\prime} .
$$

Proof. Since $x^{-1} \operatorname{Diff}_{\mathrm{e}}^{1}(M) \subset x^{-1} \operatorname{Diff}_{\mathrm{b}}^{1}(M),[Q, A] \in x^{-1} \Psi_{\mathrm{b}}^{m}(M)$, so (8.1) holds with $A^{\prime}=[Q, A] x \in \Psi_{\mathrm{b}}^{m}(M)$ and $Q^{\prime}=x^{-1} \in x^{-1} \operatorname{Diff}_{\mathrm{e}}^{1}(M)$. 
In fact the span of products on the left in (8.1), and hence on the right, may be characterized as the subspace of $x^{-1} \Psi_{\mathrm{b}}^{m+1}(M)$ fixed by the vanishing of the principal symbol at $x=0, \sigma=0, \zeta=0$. Similarly one may consider higher order products.

Definition 8.2. Let

$$
x^{-k} \operatorname{Diff}_{\mathrm{e}}^{k} \Psi_{\mathrm{b}}^{m}(M) \subset x^{-k} \Psi_{\mathrm{b}}^{k+m}(M)
$$

be the span of the products $Q A$ with $Q \in x^{-k} \operatorname{Diff}_{\mathrm{e}}^{k}(M)$ and $A \in \Psi_{\mathrm{b}}^{m}(M)$.

Induction based on Lemma 8.1 gives

Corollary 8.3. The space $x^{-k} \operatorname{Diff}_{\mathrm{e}}^{k} \Psi_{\mathrm{b}}^{m}(M)$ is also the span of the products $A Q$ with $Q \in x^{-k} \operatorname{Diff}_{\mathrm{e}}^{k}(M)$ and $A \in \Psi_{\mathrm{b}}^{m}(M)$ and hence

$$
\bigcup_{k, m} x^{-k} \operatorname{Diff}_{\mathrm{e}}^{k} \Psi_{\mathrm{b}}^{m}(M)
$$

forms a bigraded ring which is closed under adjoints with respect to any $b$-density.

Next consider the Hamilton vector field of the principal symbol of an element of $\Psi_{\mathrm{b}}^{m}(M)$.

Lemma 8.4. If $A \in \Psi_{\mathrm{b}}^{m}(M)$ then the Hamilton vector field $H_{a}$ of $a=$ $\sigma_{\mathrm{b}, m}(A)$, defined initially on $T^{*} M^{\circ}$, extends to an element of $\mathcal{V}_{\mathrm{b}}\left({ }^{\mathrm{b}} T^{*} M\right)$ and in canonical local coordinates $(x, w, z, \underline{\xi}, \underline{\mu}, \underline{\zeta})$ on ${ }^{\mathrm{b}} T^{*} M$, induced by local coordinates $(x, w, z)$ on $M$,

$$
H_{a}=\left(\partial_{\underline{\xi}} a\right) x \partial_{x}+\left(\partial_{\underline{\mu}} a\right) \partial_{w}+\left(\partial_{\underline{\zeta}} a\right) \partial_{z}-\left(x \partial_{x} a\right) \partial_{\underline{\xi}}-\left(\partial_{w} a\right) \partial_{\underline{\mu}}-\left(\partial_{z} a\right) \partial_{\underline{\zeta}} .
$$

Proof. Let $(x, w, z, \sigma, \underline{\mu}, \underline{\zeta})$ be the canonical coordinates on $T^{*} M$ induced by local coordinates $(x, \bar{w}, \bar{z})$ on $M$. In $M^{\circ}, T_{M^{\circ}}^{*} M$ and ${ }^{\mathrm{b}} T_{M^{\circ}}^{*} M$ are naturally identified via the map

$$
\pi_{\mathrm{b}}: T^{*} M \rightarrow{ }^{\mathrm{b}} T^{*} M, \pi_{\mathrm{b}}(x, y, z, \sigma, \underline{\mu}, \underline{\zeta})=(x, w, z, x \sigma, \underline{\mu}, \underline{\zeta}) .
$$

Moreover,

$$
H_{\pi_{\mathrm{b}}^{*} a}=\partial_{\sigma} \pi_{\mathrm{b}}^{*} a \partial_{x}+\partial_{\underline{\mu}} \pi_{\mathrm{b}}^{*} a \partial_{w}+\partial_{\underline{\zeta}} \pi_{\mathrm{b}}^{*} a \partial_{z}-\partial_{x} \pi_{\mathrm{b}}^{*} a \partial_{\sigma}-\partial_{w} \pi_{\mathrm{b}}^{*} a \partial_{\underline{\mu}}-\partial_{z} \pi_{\mathrm{b}}^{*} a \partial_{\underline{\zeta}} .
$$

Since $\partial_{\sigma} \pi_{\mathrm{b}}^{*} a=x \pi_{\mathrm{b}}^{*} \partial_{\underline{\xi}} a$ and $\partial_{x} \pi_{\mathrm{b}}^{*} a=\pi_{\mathrm{b}}^{*}\left(\partial_{x}+x^{-1} \underline{\xi} \partial_{\xi}\right) a$,

$$
\begin{aligned}
H_{\pi_{\mathrm{b}}^{*} a} \pi_{\mathrm{b}}^{*} c=\pi_{\mathrm{b}}^{*} & \left(\partial_{\underline{\xi}} a\left(x \partial_{x}+\underline{\xi} \partial_{\underline{\xi}}\right) c+\partial_{\underline{\mu}} a \partial_{w} c+\partial_{\underline{\zeta}} a \partial_{z} c\right. \\
& \left.-\left(x \partial_{x} a+\underline{\xi} \partial_{\underline{\xi}} a\right) \partial_{\underline{\xi}} c-\partial_{w} a \partial_{\underline{\mu}} c-\partial_{z} a \partial_{\underline{\zeta}} c\right), \quad \text { if } c \in \mathcal{C}^{\infty}\left({ }^{\mathrm{b}} T^{*} M\right) .
\end{aligned}
$$

The terms in which both $a$ and $c$ are differentiated with respect to $\underline{\xi}$ cancel, proving the lemma.

The test operator used below has the special property that its symbol is fiber constant at the compressed cotangent bundle: 
Definition 8.5. A symbol $a \in \mathcal{C}^{\infty}\left({ }^{\mathrm{b}} T^{*} M\right)$ of order $m$ is said to be basic if it is constant on the fibers above ${ }^{\mathrm{b}} \dot{T}^{*}(M)$, i.e. in terms of local coordinates $\partial_{z}(a)=0$ at $\{x=\underline{\xi}=\underline{\zeta}=0\}$. An operator having such a principal symbol is also said to be basic.

Lemma 8.6. If $A \in \Psi_{\mathrm{b}}^{m}(M)$ there exist $B \in \Psi_{\mathrm{b}}^{m}(M), C \in \Psi_{\mathrm{b}}^{m-1}(M)$, depending continuously on $A$, such that

$$
\left[D_{x}, A\right]=B+C D_{x}
$$

with $\sigma(B)=-i \partial_{x}(\sigma(A)), \sigma(C)=-i \partial_{\underline{\xi}}(\sigma(A))$. If in addition $A$ is a basic operator,

$$
\left[x^{-1} D_{z_{i}}, A\right]=B_{i}+C_{i} D_{x}+\sum_{j} E_{i j} x^{-1} D_{z_{j}}+x^{-1} F_{i}
$$

with $B_{i} \in \Psi_{\mathrm{b}}^{m}(M), C_{i}, E_{i j}, F_{i} \in \Psi_{\mathrm{b}}^{m-1}(M)$ depending continuously on $A$ and

$$
-i \partial_{z_{i}}(\sigma(A))-i \underline{\zeta}_{j} \partial_{\underline{\xi}}\left(\sigma(A)=x \sigma\left(B_{i}\right)+\underline{\xi}_{\sigma} \sigma\left(C_{i}\right)+\sum_{j} \underline{\zeta}_{j} \sigma\left(E_{i j}\right) .\right.
$$

Proof. Writing

$$
\left[D_{x}, A\right]=\left[x^{-1}\left(x D_{x}\right), A\right]=x^{-1}\left[x D_{x}, A\right]+\left[x^{-1}, A\right] x D_{x},
$$

we recall from the end of $\S 3$ that $\left[x D_{x}, A\right]$ is one power of $x$ more regular than the commutator of two generic b-operators, i.e. $\left[x D_{x}, A\right] \in x \Psi_{\mathrm{b}}^{m}(M)$, so setting $B=x^{-1}\left[x D_{x}, A\right] \in \Psi_{\mathrm{b}}^{m}(M)$ Lemma 8.4 shows $\sigma_{m}(B)=-i x^{-1} H_{\xi} a=$ $-i \partial_{x} a$ with $a=\sigma(A)$. Moreover, $C=\left[x^{-1}, A\right] x \in \Psi_{\mathrm{b}}^{m-1}(M)$ has $\sigma_{m-1}(\bar{C})=$ $-i H_{x^{-1}} a=-i \partial_{\underline{\xi}} a$.

On the other hand, $x^{-1} D_{z_{j}} \in x^{-1} \Psi_{\mathrm{b}}^{1}(M)$, so $\left[x^{-1} D_{z_{j}}, A\right] \in x^{-1} \Psi_{\mathrm{b}}^{m}(M)$, and $\sigma\left(\left[x^{-1} D_{z_{j}}, A\right]\right)=-i\left(x^{-1} \underline{\zeta}_{j} \partial_{\underline{\xi}} a+x^{-1} \partial_{z_{j}} a\right)$. By assumption, $\partial_{z_{j}} a$ vanishes at $x=0, \underline{\zeta}=0, \underline{\xi}=0$, so we can write $\partial_{z_{j}} a=x b_{j}+\xi c_{j}+\sum_{k} \zeta_{k} e_{j k}$ with $b_{j}$ homogeneous of degree $m, c_{j}, e_{j k}$ homogeneous of degree $m-1$. Now let $B_{j} \in \Psi_{\mathrm{b}}^{m}(M)$ with $\sigma\left(B_{j}\right)=-i b_{j}, C_{j}, E_{j k} \in \Psi_{\mathrm{b}}^{m-1}(M)$ with $\sigma\left(C_{j}\right)=-i c_{j}$, $\sigma\left(E_{j k}\right)=-i\left(e_{j k}+\delta_{j k} \partial_{\xi} a\right)$. Then $R=\left[x^{-1} D_{z_{j}}, A\right]-B_{j}-C_{j} D_{x}-\sum E_{j k} D_{z_{k}}$ satisfies $R \in x^{-1} \Psi_{\mathrm{b}}^{m}(M)$ and $\sigma(R)=0$, so $R \in x^{-1} \Psi_{\mathrm{b}}^{m-1}(M)$, i.e. $R=x^{-1} F$ with $F \in \Psi_{\mathrm{b}}^{m-1}(M)$, finishing the proof of (8.3).

Remark 8.7. Since $M$ is non-compact and the results here are microlocal, we shall fix a compact set $\tilde{K} \subset M$ and assume that all pseudodifferential operators under consideration have their Schwartz kernels supported in $\tilde{K} \times$ $\tilde{K}$. Choose $\phi \in \mathcal{C}_{c}^{\infty}(M)$ which is identically equal to 1 in a neighborhood of $\tilde{K}$ and fiber constant. Below we use the notation $\|\cdot\|_{\tilde{\mathcal{D}}_{\text {loc }}}$ for $\|\phi u\|_{\tilde{\mathcal{D}}}$ to avoid having to specify $\tilde{U}$. We also write $\|\phi v\|_{\tilde{\mathcal{D}}^{\prime}}$ as $\|v\|_{\tilde{\mathcal{D}}_{\text {loc }}^{\prime}}$.

Next comes the crucial estimate. 
Lemma 8.8. Suppose that $K \subset U \subset{ }^{\mathrm{b}} S^{*} X$ with $K$ compact and $U$ open and that $\mathcal{A}=\left\{A_{r} \in \Psi_{\mathrm{b}}^{s-1}(X): r \in(0,1]\right\}$ a basic family with $\mathrm{WF}_{\mathrm{b}}^{\prime}(\mathcal{A}) \subset$ $K$ which is bounded in $\Psi_{\mathrm{b} \infty}^{s}(X)$. Then there exist $G \in \Psi_{\mathrm{b}}^{s-1 / 2}(X), \tilde{G} \in$ $\Psi_{\mathrm{b}}^{s+1 / 2}(X)$ with $\mathrm{WF}_{\mathrm{b}}^{\prime}(G), \mathrm{WF}_{\mathrm{b}}^{\prime}(\tilde{G}) \subset U$ and $C_{0}>0$ such that

$$
\begin{aligned}
& \left|\int_{M}\left(\left|d_{X} A_{r} u\right|^{2}-\left|D_{t} A_{r} u\right|^{2}\right)\right| \\
& \leq C_{0}\left(\|u\|_{\tilde{\mathcal{D}}_{l o c}}^{2}+\|G u\|_{\tilde{\mathcal{D}}^{\prime}}^{2}+\|\square u\|_{\tilde{\mathcal{D}}_{l o c}^{\prime}}^{2}+\|\tilde{G} \square u\|_{\tilde{\mathcal{D}}^{\prime}}^{2}\right), \\
& \forall r \in(0,1], u \in H_{0, l o c}^{1}(X) \\
& \text { with } \mathrm{WF}_{\mathrm{b}, \tilde{\mathcal{D}}}^{s-1 / 2}(u) \cap U=\emptyset, \mathrm{WF}_{\mathrm{b}, \tilde{\mathcal{D}}^{\prime}}^{s+1 / 2}(\square u) \cap U=\emptyset
\end{aligned}
$$

where the meaning of $\|u\|_{\tilde{\mathcal{D}}_{\text {loc }}}^{2}$ and $\|\square u\|_{\tilde{\mathcal{D}}_{\text {loc }}^{\prime}}^{2}$ is stated above in Remark 8.7.

It follows that if $\square u=0$

$$
\left|\int_{M}\left(\left|d_{X} A_{r} u\right|^{2}-\left|D_{t} A_{r} u\right|^{2}\right)\right| \leq C_{0}\left(\|u\|_{\tilde{\mathcal{D}}_{\text {loc }}}^{2}+\|G u\|_{\tilde{\mathcal{D}}}^{2}\right) .
$$

The main point of this lemma is that $G$ is $1 / 2$ order lower than the family $\mathcal{A}$. In the limit, $r \rightarrow 0$, this gives control of the Dirichlet form evaluated on $A_{0} u, A_{0} \in \Psi_{\mathrm{b} \infty}^{s}(M)$, in terms of lower order information. The role of $A_{r}$ for $r>0$ is to regularize such an argument, i.e. to ensure that the terms in a formal computation, in which one uses $A_{0}$ directly, actually make sense.

Proof. The assumption on the wavefront set of $u$ implies that $A_{r} u \in \tilde{\mathcal{D}}$ for $r \in(0,1]$, so, writing $\langle\cdot, \cdot\rangle$ for the pairing in $L^{2}(M)$,

$$
\left\|d_{X} A_{r} u\right\|_{L^{2}(M)}^{2}-\left\|D_{t} A_{r} u\right\|_{L^{2}(M)}^{2}=-\left\langle\square A_{r} u, A_{r} u\right\rangle .
$$

Here the right hand side is the pairing of $\tilde{\mathcal{D}}^{\prime}$ with $\tilde{\mathcal{D}}$. Writing $\square A_{r}=A_{r} \square+$ $\left[\square, A_{r}\right]$, the right hand side can be estimated by

$$
\left|\left\langle A_{r} \square u, A_{r} u\right\rangle\right|+\left|\left\langle\left[\square, A_{r}\right] u, A_{r} u\right\rangle\right| .
$$

The lemma is therefore proved once it is shown that the first term of (8.5) is bounded by

$$
C_{0}^{\prime}\left(\|u\|_{\tilde{\mathcal{D}}_{\text {loc }}}^{2}+\|G u\|_{\tilde{\mathcal{D}}}^{2}+\|\square u\|_{\tilde{\mathcal{D}}_{\text {loc }}^{\prime}}^{2}+\|\tilde{G} \square u\|_{\tilde{\mathcal{D}}^{\prime}}^{2}\right),
$$

and the second term is bounded by $C_{0}^{\prime \prime}\left(\|u\|_{\tilde{\mathcal{D}}_{\text {loc }}}^{2}+\|G u\|_{\tilde{\mathcal{D}}}^{2}\right)$.

The first estimate is straightforward. Let $\Lambda_{-1 / 2} \in \Psi_{\mathrm{b}}^{-1 / 2}(M)$ be elliptic with $\Lambda_{1 / 2} \in \Psi_{\mathrm{b}}^{1 / 2}(M)$ a parametrix (hence also elliptic), so

$$
E=\Lambda_{-1 / 2} \Lambda_{1 / 2}-\mathrm{Id}, \quad E^{\prime}=\Lambda_{1 / 2} \Lambda_{-1 / 2}-\mathrm{Id} \in \Psi_{\mathrm{b}}^{-\infty}(M) .
$$

Then

$$
\begin{aligned}
\left\langle A_{r} \square u, A_{r} u\right\rangle & =\left\langle\left(\Lambda_{-1 / 2} \Lambda_{1 / 2}-E\right) A_{r} \square u, A_{r} u\right\rangle \\
& =\left\langle\Lambda_{1 / 2} A_{r} \square u, \Lambda_{-1 / 2}^{*} A_{r} u\right\rangle-\left\langle A_{r} \square u, E^{*} A_{r} u\right\rangle .
\end{aligned}
$$


Since $\Lambda_{1 / 2} A_{r}$ is uniformly bounded in $\Psi_{\mathrm{b} \infty}^{s+1 / 2}(M)$, and $\Lambda_{-1 / 2}^{*} A_{r}$ is uniformly bounded in $\Psi_{\mathrm{b} \infty}^{s-1 / 2}(M),\left\langle\Lambda_{1 / 2} A_{r} \square u, \Lambda_{-1 / 2}^{*} A_{r} u\right\rangle$ is uniformly bounded, with a bound like (8.6) using Cauchy-Schwarz and Lemma 6.8. Indeed, using Lemma 6.8 , choosing any $G \in \Psi_{\mathrm{b}}^{s-1 / 2}(M)$ which is elliptic on $K$, there is a constant $C_{1}>0$ such that

$$
\left\|\Lambda_{-1 / 2}^{*} A_{r} u\right\|_{\tilde{\mathcal{D}}}^{2} \leq C_{1}\left(\|u\|_{\tilde{\mathcal{D}}_{\mathrm{loc}}}^{2}+\|G u\|_{\tilde{\mathcal{D}}}^{2}\right)
$$

Similarly, by Lemma 6.8 (or more precisely, its version with $\mathrm{WF}_{\mathrm{b}, \tilde{\mathcal{D}}^{\prime}}$ ) choosing any $\tilde{G} \in \Psi_{\mathrm{b}}^{s+1 / 2}(M)$ which is elliptic on $K$, there is a constant $C_{1}^{\prime}>0$ such that $\left\|\Lambda_{1 / 2} A_{r} \square u\right\|_{\tilde{\mathcal{D}}^{\prime}}^{2} \leq C_{1}^{\prime}\left(\|\square u\|_{\tilde{\mathcal{D}}_{\text {loc }}^{\prime}}^{2}+\|\tilde{G} \square u\|_{\tilde{\mathcal{D}}^{\prime}}^{2}\right)$. Combining these gives, with $C_{0}^{\prime}=C_{1}+C_{1}^{\prime}$,

$$
\begin{aligned}
\left|\left\langle\Lambda_{1 / 2} A_{r} \square u, \Lambda_{-1 / 2}^{*} A_{r} u\right\rangle\right| & \leq\left\|\Lambda_{1 / 2} A_{r} \square u\right\|\left\|\Lambda_{-1 / 2}^{*} A_{r} u\right\| \\
\leq & \left\|\Lambda_{1 / 2} A_{r} \square u\right\|^{2}+\left\|\Lambda_{-1 / 2}^{*} A_{r} u\right\|^{2} \\
& \leq C_{0}^{\prime}\left(\|u\|_{\tilde{\mathcal{D}}_{\text {loc }}}^{2}+\|G u\|_{\tilde{\mathcal{D}}^{2}}^{2}+\|\square u\|_{\tilde{\mathcal{D}}_{\text {loc }}^{\prime}}^{2}+\|\tilde{G} \square u\|_{\tilde{\mathcal{D}}^{\prime}}^{2}\right),
\end{aligned}
$$

as desired.

A similar argument using the assumption that $A_{r}$ is uniformly bounded in $\Psi_{\mathrm{b} \infty}^{s}(M)$, and the uniform boundedness of $E^{*} A_{r}$ in $\Psi_{\mathrm{b} \infty}^{s-1 / 2}(M)$ (in fact it is bounded in $\Psi_{\mathrm{b} \infty}^{-\infty}(M)$, ) shows that $\left\langle A_{r} \square u, E^{*} A_{r} u\right\rangle$ is uniformly bounded.

Now we turn to the second term in (8.5). Let $Q_{i} \in x^{-1} \operatorname{Diff}_{\mathrm{e}}^{1}(M)$ be a local basis of $x^{-1} \operatorname{Diff}_{\mathrm{e}}^{1}(M)$ as a $\mathcal{C}^{\infty}(M)$-module. Using Lemma 8.6,

$$
\left[\square, A_{r}\right]=\sum_{i, j} Q_{i} Q_{j} B_{i j, r}^{\prime}+\sum Q_{j} B_{j, r}^{\prime}+B_{r}^{\prime}
$$

$B_{r}^{\prime} \in \Psi_{\mathrm{b}}^{s}(M), B_{j, r}^{\prime} \in \Psi_{\mathrm{b}}^{s-1}(M), B_{i j, r}^{\prime} \in \Psi_{\mathrm{b}}^{s-2}(M)$, uniformly bounded in $\Psi_{\mathrm{b} \infty}^{s+1}(M)$, resp. $\Psi_{\mathrm{b} \infty}^{s}(M)$, resp. $\Psi_{\mathrm{b} \infty}^{s-1}(M)$. With $\Lambda_{-1 / 2} \in \Psi_{\mathrm{b}}^{-1 / 2}(M)$ as above, using Lemma 8.1, we can write further

$$
\Lambda_{1 / 2}\left[\square, A_{r}\right]=\sum_{i, j} Q_{i} Q_{j} B_{i j, r}+\sum Q_{j} B_{j, r}+B_{r}
$$

with $B_{r}$, etc, as their primed analogues, but of order $1 / 2$ greater: $B_{r} \in$ $\Psi_{\mathrm{b}}^{s+1 / 2}(M), B_{j, r} \in \Psi_{\mathrm{b}}^{s-1 / 2}(M), B_{i j, r} \in \Psi_{\mathrm{b}}^{s-3 / 2}(M)$, uniformly bounded in 
$\Psi_{\mathrm{b} \infty}^{s+3 / 2}(M), \operatorname{resp} . \Psi_{\mathrm{b} \infty}^{s+1 / 2}(M)$, resp. $\Psi_{\mathrm{b} \infty}^{s-1 / 2}(M)$. Thus,

$$
\begin{aligned}
\left\langle\left[\square, A_{r}\right] u, A_{r} u\right\rangle= & \sum_{i j}\left\langle Q_{i} Q_{j} B_{i j, r} u, \Lambda_{-1 / 2}^{*} A_{r} u\right\rangle-\sum_{i j}\left\langle Q_{i} Q_{j} E B_{i j, r} u, A_{r} u\right\rangle \\
& +\sum_{j}\left\langle Q_{j} B_{j, r} u, \Lambda_{-1 / 2}^{*} A_{r} u\right\rangle-\sum_{j}\left\langle Q_{j} E B_{j, r} u, A_{r} u\right\rangle \\
& +\left\langle B_{r} u, \Lambda_{-1 / 2}^{*} A_{r} u\right\rangle-\left\langle E B_{r} u, A_{r} u\right\rangle \\
= & \sum_{i j}\left\langle Q_{j} B_{i j, r} u, Q_{i}^{*} \Lambda_{-1 / 2}^{*} A_{r} u\right\rangle-\sum_{i j}\left\langle Q_{j} E B_{i j, r} u, Q_{i}^{*} A_{r} u\right\rangle \\
& +\sum_{j}\left\langle B_{j, r} u, Q_{j}^{*} \Lambda_{-1 / 2}^{*} A_{r} u\right\rangle-\sum_{j}\left\langle E B_{j, r} u, Q_{j}^{*} A_{r} u\right\rangle \\
& +\left\langle B_{r} u, \Lambda_{-1 / 2}^{*} A_{r} u\right\rangle-\left\langle E B_{r} u, A_{r} u\right\rangle,
\end{aligned}
$$

where $Q_{i}^{*} \in x^{-1} \operatorname{Diff}_{\mathrm{e}}^{1}(M)$ is the formal adjoint of $Q_{i}$ with respect to $d g$, and where in the last step we used

$$
B_{i j, r} u, \Lambda_{-1 / 2}^{*} A_{r} u, E B_{i j, r} u, A_{r} u \in \tilde{\mathcal{D}} .
$$

We estimate the term

$$
\left|\left\langle Q_{j} B_{i j, r} u, Q_{i}^{*} \Lambda_{-1 / 2}^{*} A_{r} u\right\rangle\right|
$$

by Cauchy-Schwarz: both factors are uniformly bounded for $r \in(0,1]$ since $\Lambda_{-1 / 2}^{*} A_{r}, B_{i j, r}$ are uniformly bounded in $\Psi_{\mathrm{b} \infty}^{s-1 / 2}(M)$ with a uniform wave front bound disjoint from $\mathrm{WF}_{\mathrm{b}, \tilde{\mathcal{D}}}^{s-1 / 2}(u)$. Indeed, as noted above, by Lemma 6.8 , choosing any $G \in \Psi_{\mathrm{b}}^{s-1 / 2}(M)$ which is elliptic on $K$, there is a constant $C_{1}>0$ such that this term is bounded by $C_{1}\left(\|u\|_{\tilde{\mathcal{D}}_{\text {loc }}}^{2}+\|G u\|_{\tilde{\mathcal{D}}}^{2}\right)$. Similar estimates apply to the other terms on the right hand side of (8.7) (with the slight technical point that for the penultimate term one uses the pairing between $\tilde{\mathcal{D}}^{-1}$ and $\left.\tilde{\mathcal{D}}\right)$, showing that $\left\langle\left[\square, A_{r}\right] u, A_{r} u\right\rangle$ is uniformly bounded for $r \in(0,1]$, indeed is bounded by $C_{0}\left(\|u\|_{\tilde{\mathcal{D}}_{\text {loc }}}^{2}+\|G u\|_{\tilde{\mathcal{D}}}^{2}\right)$, proving the lemma.

Next we refine Lemma 8.8 by including a second parameter and arranging that $\tilde{G}$ have order $s$ as opposed to $s+1 / 2$.

Lemma 8.9. Under the same hypotheses as Lemma 8.8 there exist $G \in$ $\Psi_{\mathrm{b}}^{s-1 / 2}(X)$ and $\tilde{G} \in \Psi_{\mathrm{b}}^{s}(X)$ with $\mathrm{WF}_{\mathrm{b}}^{\prime}(G), \mathrm{WF}_{\mathrm{b}}^{\prime}(\tilde{G}) \subset U$ and $C_{0}>0$ such that for $\epsilon>0, r \in(0,1]$ and $u \in \tilde{\mathcal{D}}_{l o c}$ with $\mathrm{WF}_{\mathrm{b}, \tilde{\mathcal{D}}}^{s-1 / 2}(u) \cap U=\emptyset$, $\mathrm{WF}_{\mathrm{b}, \tilde{\mathcal{D}}^{\prime}}^{s}(\square u) \cap U=\emptyset$

$$
\begin{gathered}
\left|\int_{M}\left(\left|d_{X} A_{r} u\right|^{2}-\left|D_{t} A_{r} u\right|^{2}\right)\right| \leq \epsilon \\
d_{M} A_{r} u \|_{L^{2}(M)}^{2}+C_{0}\left(\|u\|_{\tilde{\mathcal{D}}_{l o c}}^{2}+\|G u\|_{\tilde{\mathcal{D}}}^{2}\right. \\
\left.+\epsilon^{-1}\|\square u\|_{\tilde{\mathcal{D}}_{l o c}^{\prime}}^{2}+\epsilon^{-1}\|\tilde{G} \square u\|_{\tilde{\mathcal{D}}^{\prime}}^{2}\right) .
\end{gathered}
$$


Proof. It is only necessary to treat the term $\left|\left\langle A_{r} \square u, A_{r} u\right\rangle\right|$ slightly differently, using Cauchy-Schwarz:

$$
\left|\left\langle A_{r} \square u, A_{r} u\right\rangle\right| \leq\left\|A_{r} \square u\right\|_{\tilde{\mathcal{D}}^{\prime}}\left\|A_{r} u\right\|_{\tilde{\mathcal{D}}} \leq \epsilon\left\|A_{r} u\right\|_{\tilde{\mathcal{D}}^{2}}^{2}+\epsilon^{-1}\left\|A_{r} \square u\right\|_{\tilde{\mathcal{D}}^{\prime}}^{2} .
$$

Now the lemma follows by using Lemma 6.8. Definition 6.5, namely choosing any $\tilde{G} \in \Psi_{\mathrm{b}}^{s}(M)$ which is elliptic on $K$, there is a constant $C_{1}^{\prime}>0$ such that $\left\|A_{r} \square u\right\|_{\tilde{\mathcal{D}}^{\prime}}^{2} \leq C_{1}^{\prime}\left(\|\square u\|_{\tilde{\mathcal{D}}_{\text {loc }}^{\prime}}^{2}+\|\tilde{G} \square u\|_{\tilde{\mathcal{D}}^{\prime}}^{2}\right)$; the end of the proof follows that of Lemma 8.8.

Proposition 8.10. (Microlocal elliptic regularity.) If $u \in \tilde{\mathcal{D}}_{l o c}$ then

$$
\mathrm{WF}_{\mathrm{b}, \tilde{\mathcal{D}}}^{m}(u) \subset \mathrm{WF}_{\mathrm{b}, \tilde{\mathcal{D}}^{\prime}}^{m}(\square u) \cup \dot{\pi}\left({ }^{\mathrm{e}} S^{*} M\right) \text {, and } \mathrm{WF}_{\mathrm{b}, \tilde{\mathcal{D}}}^{m}(u) \cap \dot{\mathcal{E}} \subset \mathrm{WF}_{\mathrm{b}, \tilde{\mathcal{D}}^{\prime}}^{m}(\square u) \text {. }
$$

Proof. We first prove a slightly weaker result in which $\mathrm{WF}_{\mathrm{b}, \tilde{\mathcal{D}}^{\prime}}^{m}(\square u)$ is replaced by $\mathrm{WF}_{\mathrm{b}, \tilde{\mathcal{D}}^{\prime}}^{m+1 / 2}(\square u)$-we rely on Lemma 8.8. We then prove the original statement using Lemma 8.9.

Suppose that either $q \in{ }^{\mathrm{b}} \dot{S}^{*} M \backslash \dot{\pi}\left({ }^{\mathrm{e}} S^{*} M\right)$ or $q \in \dot{\mathcal{E}}$. We may assume iteratively that $q \notin \mathrm{WF}_{\mathrm{b}, \tilde{\mathcal{D}}}^{s-1 / 2}(u)$; we need to prove then that $q \notin \mathrm{WF}_{\mathrm{b}, \tilde{\mathcal{D}}}^{s}(u)$ (note that the inductive hypothesis holds for $s=1 / 2$ since $u \in \tilde{\mathcal{D}}_{\text {loc }}$ ). Let $A \in \Psi_{\mathrm{b}}^{s}(M)$ be basic and such that $\mathrm{WF}_{\mathrm{b}}^{\prime}(A) \cap \mathrm{WF}_{\mathrm{b}, \tilde{\mathcal{D}}}^{s-1 / 2}(u)=\emptyset, \mathrm{WF}_{\mathrm{b}}^{\prime}(A) \cap$ $\mathrm{WF}_{\mathrm{b}, \tilde{\mathcal{D}}^{\prime}}^{s+1 / 2}(\square u)=\emptyset$, with $\mathrm{WF}_{\mathrm{b}}^{\prime}(A)$ in a small neighborhood $U$ of $q$ so that for a suitable $C>0$ or $\epsilon>0$, in $U$

(1) $1<C\left(\underline{\hat{\xi}}^{2}+|\underline{\hat{\zeta}}|^{2}\right)$ if $q \in{ }^{\mathrm{b}} \dot{S}^{*} M \backslash \dot{\pi}\left({ }^{\mathrm{e}} S^{*} M\right)$, or

(2) $|\underline{\hat{\xi}}|+|\underline{\hat{\zeta}}|<\epsilon\left(1+|\underline{\hat{\eta}}|^{2}\right)^{1 / 2}$, and $\hat{\eta}>1+\epsilon$, if $q \in \dot{\mathcal{E}}$.

Let $\Lambda_{r} \in \Psi_{\mathrm{b}}^{-2}(M)$ for $r>0$, such that $\mathcal{L}=\left\{\Lambda_{r}: r \in(0,1]\right\}$ is a bounded family in $\Psi_{\mathrm{b}}^{0}(M)$, and $\Lambda_{r} \rightarrow \mathrm{Id}$ as $r \rightarrow 0$ in $\Psi_{\mathrm{b}}^{\tilde{\epsilon}}(M), \tilde{\epsilon}>0$; we let the symbol of $\Lambda_{r}$ be $\left(1+r\left(\underline{\tau}^{2}+|\underline{\eta}|^{2}+|\underline{\zeta}|^{2}+|\underline{\xi}|^{2}\right)\right)^{-1}$. Let $A_{r}=\Lambda_{r} A$. Let $a$ be the symbol of $A$, hence for $r>\overline{0}$,

$$
\sigma\left(A_{r}\right)=\left(1+r\left(\underline{\tau}^{2}+|\underline{\eta}|^{2}+|\underline{\zeta}|^{2}+|\underline{\xi}|^{2}\right)\right)^{-1} a .
$$

We now have $A_{r} \in \Psi_{\mathrm{b}}^{s-2}(M)$ for $r>0$, and $A_{r}$ is uniformly bounded in $\Psi_{\mathrm{b} \infty}^{s}(M), A_{r} \rightarrow A$ in $\Psi_{\mathrm{b} \infty}^{s+\tilde{\epsilon}}(M)$.

By Lemma 8.8,

$$
\left\|d_{X} A_{r} u\right\|^{2}-\left\|D_{t} A_{r} u\right\|^{2}
$$

is uniformly bounded for $r \in(0,1]$. Write the dual metric as

$$
\begin{aligned}
g^{-1}=\partial_{x}^{2}+2 & \sum_{i} B_{i} \partial_{x} \partial_{y_{i}}+2 \sum_{i} C_{i} \partial_{x}\left(x^{-1} \partial_{z_{i}}\right)+\sum_{i, j} B_{i j} \partial_{y_{i}} \partial_{y_{j}} \\
& +\sum_{i, j} C_{i j}\left(x^{-1} \partial_{z_{i}}\right)\left(x^{-1} \partial_{z_{j}}\right)+2 \sum_{i, j} E_{i j} \partial_{y_{i}}\left(x^{-1} \partial_{z_{j}}\right)
\end{aligned}
$$


Then

$$
\begin{gathered}
\int_{M}\left|d_{X} A_{r} u\right|^{2} d g=\int_{M}\left|D_{x} A_{r} u\right|^{2} d g+\int_{M} \sum B_{i} D_{x} A_{r} u \overline{D_{y_{i}} A_{r} u} d g \\
+\int_{M} \sum C_{i} D_{x} A_{r} u \overline{x^{-1} D_{z_{i}} A_{r} u} d g+\int_{M} \sum B_{i j} D_{y_{i}} A_{r} u \overline{D_{y_{j}} A_{r} u} d g \\
+\int_{M} \sum C_{i j} x^{-1} D_{z_{i}} A_{r} u \overline{x^{-1} D_{z_{j}} A_{r} u} d g+\int_{M} \sum E_{i j} D_{y_{i}} A_{r} u \overline{x^{-1} D_{z_{j}} A_{r} u} d g .
\end{gathered}
$$

As $B_{i j}(x, y, z)=B_{i j}(0, y, z)+x B_{i j}^{\prime}(x, y, z)$ (in fact, $B(0, y, z)$ is independent of $z$ ), we see that if $A_{r}$ is supported in $x<\delta$,

$$
\left|\int_{M} x B_{i j}^{\prime} D_{y_{i}} A_{r} u \overline{D_{y_{j}} A_{r} u} d g\right| \leq C \delta \sum_{i^{\prime}, j^{\prime}}\left\|D_{y_{i^{\prime}}} A_{r} u\right\|\left\|D_{y_{j^{\prime}}} A_{r} u\right\|,
$$

with analogous estimates for $C_{i j}(x, y, z)-C_{i j}(0, y, z)$ and for $B_{i}(x, y, z)$, $C_{i}(x, y, z)$ and $E_{i j}(x, y, z)$. Moreover, as the matrix $B_{i j}(0, y, z)$ is positive definite, for some $c>0$,

$$
c \int_{M} \sum_{j}\left|x^{-1} D_{z_{j}} A_{r} u\right|^{2} d g \leq \frac{1}{2} \int_{M} \sum_{i j} B_{i j}(0, y, z) x^{-1} D_{z_{i}} A_{r} u \overline{x^{-1} D_{z_{j}} A_{r} u} d g
$$

we also make $c<1 / 2$. Thus, there exists $\tilde{C}>0$ and $\delta_{0}>0$ such that if $\delta<\delta_{0}$ and $A$ is supported in $x<\delta$ then

$$
\begin{gathered}
c \int_{M}\left(\left|D_{x} A_{r} u\right|^{2}+\sum_{j}\left|x^{-1} D_{z_{j}} A_{r} u\right|^{2}\right) d g \\
+\int_{M}\left((1-\tilde{C} \delta) \sum_{j}\left|D_{y_{j}} A_{r} u\right|_{h}^{2}-\left|D_{t} A_{r} u\right|^{2}\right) d g \\
\leq \int_{M}\left(\left|d_{X} A_{r} u\right|^{2}-\left|D_{t} A_{r} u\right|^{2}\right) d g
\end{gathered}
$$

where we used the notation

$$
\sum_{j}\left|D_{y_{j}} A_{r} u\right|_{h}^{2}=\sum_{i j} B_{i j}(0, y, z) D_{y_{i}} A_{r} u \overline{D_{y_{j}} A_{r} u}
$$

i.e. $h$ is the dual metric $g$ restricted to the span of the $d y_{j}, j=1, \ldots, l$.

Now we distinguish the cases $q \in \dot{\mathcal{E}}$ and $q \in{ }^{\mathrm{b}} \dot{S}^{*} M \backslash \dot{\pi}\left({ }^{\mathrm{e}} S^{*} M\right)$. If $q \in \dot{\mathcal{E}}, A$ is supported near $\dot{\mathcal{E}}$, we choose $\delta \in\left(0,(2 \tilde{C})^{-1}\right)$ so that $(1-\tilde{C} \delta) \hat{\eta}^{2}>1+\delta$ on a neighborhood of $\mathrm{WF}_{\mathrm{b}}^{\prime}(A)$, which is possible in view of $(2)$ at the beginning of the proof. Then the second integral on the left hand side of (8.9) can be written as $\left\|B A_{r} u\right\|^{2}$, with the symbol of $B$ given by $\left((1-\tilde{C} \delta)|\underline{\eta}|^{2}-\underline{\tau}^{2}\right)^{1 / 2}$ (which is $\geq \delta \underline{\tau}$ ), modulo a term

$$
\int_{M} F A_{r} u \overline{A_{r} u}, F \in \Psi_{\mathrm{b}}^{1}(M)
$$


But this expression is uniformly bounded as $r \rightarrow 0$ by the argument above. We thus deduce that

$$
c\left(\left\|D_{x} A_{r} u\right\|^{2}+\sum_{j}\left\|x^{-1} D_{z_{j}} A_{r} u\right\|^{2}\right)+\left\|B A_{r} u\right\|^{2}
$$

is uniformly bounded as $r \rightarrow 0$.

If $q \in{ }^{\mathrm{b}} \dot{S}^{*} M \backslash \dot{\pi}\left({ }^{\mathrm{e}} S^{*} M\right)$, and $A$ is supported in $x<\delta$,

$$
\int_{M} \delta^{-2}\left|D_{z_{j}} A_{r} u\right|^{2} \leq \int_{M}\left|x^{-1} D_{z_{j}} A_{r} u\right|^{2}, \int_{M} \delta^{-2}\left|x D_{x} A_{r} u\right|^{2} \leq \int_{M}\left|D_{x} A_{r} u\right|^{2} .
$$

On the other hand, near $q \in{ }^{\mathrm{b}} \dot{S}^{*} M \backslash \dot{\pi}\left({ }^{\mathrm{e}} S^{*} M\right)$, for $\delta>0$ sufficiently small,

$$
\begin{gathered}
I=\int_{M}\left(\frac{c}{2 \delta^{2}}\left(\left|x D_{x} A_{r} u\right|^{2}+\sum_{j}\left|D_{z_{j}} A_{r} u\right|^{2}\right)-\left|D_{t} A_{r} u\right|^{2}\right) \\
=\left\|B A_{r} u\right\|^{2}+\int_{M} F A_{r} u \overline{A_{r} u},
\end{gathered}
$$

with the symbol of $B$ given by $\left(\left(c / 2 \delta^{2}\right)\left(\underline{\xi}^{2}+\sum \underline{\zeta}_{j}^{2}\right)-\underline{\tau}^{2}\right)^{1 / 2}$ (which does not vanish on $U$ for $\delta>0$ small), while $F \in \Psi_{\mathrm{b}}^{1}(X)$, so the second term on the right hand side is uniformly bounded as $r \rightarrow 0$. Now the LHS of (8.9) is bounded below by

$$
I+\frac{c}{2}\left(\left\|D_{x} A_{r} u\right\|^{2}+\sum_{j}\left\|x^{-1} D_{z_{j}} A_{r} u\right\|^{2}\right) .
$$

We thus deduce in this case that

$$
\frac{c}{2}\left(\left\|D_{x} A_{r} u\right\|^{2}+\sum_{j}\left\|x^{-1} D_{z_{j}} A_{r} u\right\|^{2}\right)+\left\|B A_{r} u\right\|^{2}
$$

is uniformly bounded as $r \rightarrow 0$.

We thus conclude that $D_{x} A_{r} u, x^{-1} D_{z_{j}} A_{r} u, B A_{r} u$ are uniformly bounded $L^{2}(M)$. Correspondingly there are sequences $D_{x} A_{r_{k}} u, x^{-1} D_{z_{j}} A_{r_{k}} u, B A_{r_{k}} u$, weakly convergent in $L^{2}(M)$, and such that $r_{k} \rightarrow 0$, as $k \rightarrow \infty$. Since they converge to $D_{x} A u, x^{-1} D_{z_{j}} A u, B A u$, respectively, in $\mathcal{C}^{-\infty}(M)$, we deduce that the weak limits are $D_{x} A u, x^{-1} D_{z_{j}} A u, B A u$, which therefore lie in $L^{2}(M)$. Consequently, $d A u \in L^{2}(M)$ proving the proposition with $\mathrm{WF}_{\mathrm{b}, \tilde{\mathcal{D}}^{\prime}}^{m}(\square u)$ replaced by $\mathrm{WF}_{\mathrm{b}, \tilde{\mathcal{D}}^{\prime}}^{m+1 / 2}(\square u)$.

To obtain the full result observe from Lemma 8.9 that for any $\epsilon>0$

$$
\begin{aligned}
\int_{M} & \left(\left|d_{X} A_{r} u\right|^{2}-\left|D_{t} A_{r} u\right|^{2}-\epsilon\left|d_{M} A_{r} u\right|^{2}\right) \\
& =\int_{M}\left((1-\epsilon)\left|d_{X} A_{r} u\right|^{2}-(1+\epsilon)\left|D_{t} A_{r} u\right|^{2}\right)
\end{aligned}
$$


is uniformly bounded above for $r \in(0,1]$. By repeating the argument for sufficiently small $\epsilon>0$, the right hand side gives an upper bound for

$$
\frac{c}{2} \int_{X}\left(\left|D_{x} A_{r} u\right|^{2}+\sum_{j}\left|x^{-1} D_{z_{j}} A_{r} u\right|^{2}\right)+\left\|B A_{r} u\right\|^{2},
$$

which is thus uniformly bounded as $r \rightarrow 0$ and the result follows.

Theorem 8.11. (Elliptic regularity) Suppose $\square u=0$. Then $\mathrm{WF}_{\mathrm{b}, \tilde{\mathcal{D}}}(u) \subset$ $\dot{\Sigma}$.

Proof. Over $M^{\circ}$, this is ordinary elliptic regularity. Over $\partial M$, we note that it follows from the first part of Proposition 8.10 that the wavefront set is contained in $\dot{\pi}\left({ }^{\mathrm{e}} S^{*} M\right)$. On the other hand, $\dot{\pi}\left({ }^{\mathrm{e}} S^{*} M\right) \backslash \dot{\Sigma}=\dot{\mathcal{E}}$, so the result then follows from the second part of the proposition.

\section{LAW OF REFLECTION}

In this section we show that singularities interacting with the boundary may be microlocalized to give a propagation theorem in the slow variables, i.e. constraining the values of $t, y$ and their dual variables at which singularities come off the boundary. The argument is global in the fast variables in the fiber (and in a certain sense the dual to the normal variable is also 'fast'). Recall that we may freely regard $\mathrm{WF}_{\mathrm{b}, \tilde{\mathcal{D}}}(u)$ as a subset of ${ }^{\mathrm{b}} S^{*} M$ or of ${ }^{\mathrm{b}} \dot{S}^{*} M$ (see Definition 6.10 ).

As is already clear from [19], subprincipal terms matter in the commutator argument, so our arguments are somewhat delicate. To carry out commutator computations we trivialize the fibration locally near a point of $Y$, and hence extend the trivialization to a neighborhood in $M$ of the fiber above $y$. Thus an open set $O$ in $M$ is identified with $[0, \epsilon)_{x} \times O^{\prime} \times Z$, where $O^{\prime} \ni y$ is open in $Y$ and we take $O^{\prime}$ to be a coordinate patch. Then ${ }^{\mathrm{b}} T_{O}^{*} M$ is identified with ${ }^{\mathrm{b}} T^{*}\left([0, \epsilon) \times O^{\prime}\right) \times T^{*} Z$, and this product decomposition allows us to pull back functions from ${ }^{\mathrm{b}} T^{*}\left([0, \epsilon) \times O^{\prime}\right)$ to ${ }^{\mathrm{b}} T_{O}^{*} M$.

In particular this gives a connection for the fibration near the chosen fiber. Any b-vector field $V$ on $[0, \epsilon) \times O^{\prime}$ lifts to $O$ via the product identification and then for any vector field $W$ tangent to the fibers, the commutator $[V, W]$ is also tangent to the fibers. Indeed, if $W$ is the lift of a vector field from $Z$, the conclusion is clear, and in general $W$ is a finite linear combination of such vector fields with coefficients on $M$. If $W$ is only tangent to the fibers over the boundary then the same is true of $[V, W]$. Also, if $f$ is the pull-back of a function on $[0, \epsilon) \times O^{\prime}$, its commutator with $W$ vanishes. Using this local trivialization of the fibration we also choose an explicit quantization map for b-pseudodifferential operators.

Definition 9.1. Fix a partition of unity $\phi_{i}$ on $Z$ supported in coordinate charts, cut-offs $\chi_{i}$ identically equal to 1 on a neighborhood of $\operatorname{supp} \phi_{i}$ and 
still supported in coordinate charts and a function $\rho \in \mathcal{C}_{c}^{\infty}((-1 / 2,1 / 2))$ identically 1 near 0 . Then for $a \in S^{m}\left({ }^{\mathrm{b}} T^{*} O\right)$ set

$$
\begin{aligned}
& \operatorname{Op}_{\text {trv }}(a)=\sum_{i} \chi_{i} A_{i} \chi_{i}, \text { where } \\
& \begin{aligned}
A_{i}=(2 \pi)^{-n} \int e^{i\left(\underline{\xi} \cdot \frac{x-x^{\prime}}{x^{\prime}}+\underline{\eta} \cdot\left(y-y^{\prime}\right)+\underline{\zeta} \cdot\left(z-z^{\prime}\right)\right)} \\
\quad \rho\left(\frac{x-x^{\prime}}{x^{\prime}}\right) a(x, y, \underline{\xi}, \underline{\eta}, \underline{\zeta}) \phi_{i}(z) d \underline{\xi} d \underline{\zeta} d \underline{\eta} \nu .
\end{aligned}
\end{aligned}
$$

This is an explicit semi-global quantization, with $\nu$ a choice of right density, for the algebra of (compactly supported) b-pseudodifferential operators, as can be seen directly by lifting the kernels to the b-stretched product on which the cut-off $\rho\left(x / x^{\prime}-1\right)$ is smooth. In particular it is surjective, modulo terms of order $-\infty$, for operators essentially supported near the given fiber over the boundary.

To construct a 'test operator' we choose a symbol $\tilde{a}$ on ${ }^{\mathrm{b}} T^{*}\left([0, \epsilon) \times O^{\prime}\right)$, lift it to ${ }^{\mathrm{b}} T^{*} O$ (still denoting it by $\tilde{a}$ ) and multiply it by a cut-off that is identically 1 near $\dot{\Sigma}$. Thus set

$$
a=\tilde{a} \psi\left(\underline{\hat{\xi}}^{2}+|\underline{\hat{\zeta}}|^{2}\right)
$$

with $\psi \in \mathcal{C}_{c}^{\infty}(\mathbb{R})$ supported in $\left[-2 c_{1}, 2 c_{1}\right]$ and identically equal to 1 on $\left[-c_{1}, c_{1}\right]$. Thus, $d \psi\left(\underline{\hat{\xi}}^{2}+|\hat{\hat{\zeta}}|^{2}\right)$ is supported in $\underline{\hat{\xi}}^{2}+|\hat{\hat{\zeta}}|^{2} \in\left[c_{1}, 2 c_{1}\right]$ and hence outside $\dot{\Sigma}$. This may be thought of as a factor microlocalizing near the characteristic set but effectively commuting with $\square$. Note that $a$ is indeed a symbol on ${ }^{\mathrm{b}} T^{*} O$.

Lemma 9.2. With $\tilde{a} \in S^{m}\left({ }^{\mathrm{b}} T^{*}\left([0, \epsilon) \times O^{\prime}\right)\right)$ given by $(9.2), A=O p_{\operatorname{trv}}(a)$ and $W$ a vector field lifted from $Z$ using the local trivialization of the fibration,

$$
[W, A]=B \in \Psi_{\mathrm{b}}^{m}(M)
$$

has wave front set disjoint from $\dot{\Sigma}$ and depends continuously on a.

Proof. Changing $A$ by an operator of order $-\infty$ operator does not affect the conclusion, so in particular the commutator terms involving the cut-offs $\chi_{i}$ in the definition of $\mathrm{Op}_{\mathrm{trv}}(a)$ may be ignored.

Working modulo $\Psi_{\mathrm{b}}^{-\infty}(M)$, so localizing near the diagonal as necessary, the Schwartz kernel of $A$ can be computed using product coordinate charts $\tilde{O} \times \tilde{O}$. In these, where we allow coordinate changes in $z, A$ takes the form

$$
\begin{aligned}
(2 \pi)^{-n} \int e^{i\left(\underline{\xi} \cdot \frac{x-x^{\prime}}{x^{\prime}}+\underline{\eta} \cdot\left(y-y^{\prime}\right)+\underline{\zeta} \cdot\left(z-z^{\prime}\right)\right)} \\
\rho\left(\frac{x-x^{\prime}}{x^{\prime}}\right) \tilde{a}(x, y, \underline{\xi}, \underline{\eta}) \tilde{\psi}(x, y, z, \underline{\xi}, \underline{\eta}, \underline{\zeta}) d \underline{\xi} d \underline{\zeta} d \underline{\eta},
\end{aligned}
$$


where $d(\tilde{\psi})$ is supported away from $\dot{\Sigma}$. The commutator $[A, W]$ thus arises from the derivative of $\tilde{\psi}$ along $W$, hence is microsupported away from $\dot{\Sigma}$.

The adjoint of $\mathrm{Op}_{\text {trv }}(a)$ with respect to $d g$ is not quite of the form $\mathrm{Op}_{\text {trv }}(b)$ because we have not used Weyl quantization in (9.1), however it is of this form modulo terms of order $-\infty$ since $\mathrm{Op}_{\mathrm{trv}}$ is a full quantization of the b-calculus. If $\nu=\nu_{B} \nu_{Z}$ is a product type edge density relative to the trivialization, with $B=[0, \epsilon) \times O^{\prime}$, and $a$ is given by (9.2) then, modulo terms with $\mathrm{WF}_{\mathrm{b}}^{\prime}$ disjoint from $\dot{\Sigma}$ (arising from the cut-off $\psi$ ) the adjoint $\mathrm{Op}_{\text {trv }}(a)^{\dagger}$ of $A$ with respect to $\nu$ reduces to the adjoint with respect to $\nu_{B}$. Since the quantization is of product type, the full symbol of the adjoint is of the same form (9.2). Thus $A$ may be arranged to be self-adjoint with respect to $\nu$. Then $d g=J \nu$, for some smooth nonvanishing $J$, and the adjoint $A^{*}$ of $A$ with respect to $d g$ is $A^{*}=J^{-1} A^{\dagger} J$, i.e. if $A^{\dagger}=A$ then $A^{*}=J^{-1} A J$.

Lemma 9.3. If $A=A^{\dagger}$ with symbol given by (9.2) and $W$ is a vector field lifted from $Z$ using the trivialization of the fibration, then $E \in \Psi_{\mathrm{b}}^{2 m}(M)$, and $F \in \Psi_{\mathrm{b}}^{2 m-1}(M)$ may be chosen to depend continuously on $A$ so that

$$
\left[W, A^{*} A\right]=F+E, \sigma(F)=a\{a, W(\log J)\}, \mathrm{WF}_{\mathrm{b}}^{\prime}(E) \cap \dot{\Sigma}=\emptyset,
$$

where $W(\log J) \in \mathcal{C}^{\infty}(M)$ is lifted to ${ }^{\mathrm{b}} T^{*} M$ by the bundle projection.

Proof. Working modulo operators which have wavefront set disjoint from $\dot{\Sigma}$, $[A, W] \equiv 0$ so

$$
\begin{aligned}
{\left[W, A^{*} A\right] } & \equiv\left[W, J^{-1}\right] A J A+J^{-1} A[W, J] A \\
& \equiv-J^{-2}[W, J] A J A+J^{-1} A[W, J] A \equiv J^{-1}\left[A,[W, J] J^{-1}\right] J A .
\end{aligned}
$$

Then $F$ and $E$ may be defined by inserting appropriate cut-offs.

Using the chosen local trivialization of the fibration, set

$$
Q_{0}=x^{-1}, Q_{1}=D_{x} \text { and } Q_{j}=x^{-1} W_{j}, j \geq 2
$$

where the $W_{j}$ are the lifts of vector fields from $Z$ spanning $\mathcal{V}(Z)$ over $\mathcal{C}^{\infty}(Z)$.

Lemma 9.4. The $Q_{i}$ span the $\mathcal{C}^{\infty}(M)$-module $x^{\ell-1} \operatorname{Diff}_{\mathrm{e}}^{1}(M) / x^{\ell} \operatorname{Diff}_{\mathrm{b}}^{1}(M)$ over $x^{\ell} \mathcal{C}^{\infty}(M)$; in fact for every $\ell$,

$$
\begin{aligned}
P_{1} \in x^{\ell-1} \operatorname{Diff}_{\mathrm{e}}^{1}(M) \Longrightarrow \exists B_{i} \in x^{\ell} \mathcal{C}^{\infty}(M) \text { and } C & \in x^{\ell} \mathcal{V}_{\mathrm{b}}(M) \\
\text { such that } P_{1} & =\sum B_{i} Q_{i}+C .
\end{aligned}
$$

Similarly, each element $P \in x^{\ell-2} \operatorname{Diff}_{\mathrm{e}}^{2}(M)$ may be decomposed as

$$
\begin{aligned}
& P=\sum Q_{i}^{*} B_{i j} Q_{j}+\sum C_{i} Q_{i}+L, \\
& \quad \text { with } B_{i j} \in x^{\ell} \mathcal{C}^{\infty}(M), C_{i} \in x^{\ell} \mathcal{V}_{\mathrm{b}}(M) \text { and } L \in x^{\ell} \operatorname{Diff}_{\mathrm{b}}^{2}(M) .
\end{aligned}
$$


Proof. If (9.6) holds locally over a covering of $M$ by coordinate charts then summing, on the left, over a partition of unity subordinate to this cover gives the semi-global result. Thus, it suffices to work in coordinates compatible with the chosen trivialization. The vector fields $x D_{x}, x D_{y_{i}}$ and $W_{j}$ span $\mathcal{V}_{\mathrm{e}}(U)$ so if $P_{1} \in x^{\ell-1} \operatorname{Diff}_{\mathrm{e}}^{1}(M)$ then over $U, x P_{1}=a\left(x D_{x}\right)+\sum b_{j} W_{j}+$ $\sum c_{i}\left(x D_{y_{i}}\right)+c$ with $a, c, b_{j}, c_{i} \in x^{\ell-1} \mathcal{C}^{\infty}(U)$. This is the local form of (9.6):

$$
P_{1}=c Q_{0}+a Q_{1}+\sum b_{j} Q_{j}+\sum\left(c_{i} x\right) D_{y_{i}} .
$$

For the second claim, write $P=\sum P_{i}^{*} P_{i}^{\prime}$ with $P_{i} \in x^{-1} \operatorname{Diff}_{\mathrm{e}}^{1}(M), P_{i}^{\prime} \in$ $x^{\ell-1} \operatorname{Diff}_{\mathrm{e}}^{1}(M)$, and apply the first part to $P_{i}$ and $P_{i}^{\prime}$.

Lemma 9.5. With the $Q_{i}$ as in (9.5) there exist $M_{i}, N_{i} \in x \operatorname{Diff}_{\mathrm{b}}^{1}(M)$, $\kappa_{i j} \in \mathcal{C}^{\infty}(M)$ and $H \in \operatorname{Diff}_{\mathrm{b}}^{2}(M)$ such that

$$
\begin{aligned}
& -\square=\sum Q_{i}^{*} \kappa_{i j} Q_{j}+\sum Q_{i}^{*} M_{i}+\sum N_{i} Q_{i}+H \\
& \text { with }\left.\sigma(H)\right|_{x=0}=h-\underline{\tau}^{2},
\end{aligned}
$$

$h$ being the metric on the base $Y$.

Proof. If the requirements are weakened to $M_{i}, N_{i} \in \operatorname{Diff}_{\mathrm{b}}^{1}(M)$ and the conclusion regarding $\left.\sigma(H)\right|_{x=0}$ is dropped then (9.7) follows from Lemma 9.4 applied to $\square \in x^{-2} \operatorname{Diff}_{\mathrm{e}}^{2}(M)$. Moreover, every element of $x^{-1} \operatorname{Diff}_{\mathrm{e}}^{2}(M)$ has the form (9.7) with $M_{i}, N_{i}$ as stated, and with $\left.\sigma(H)\right|_{x=0}=0$. So the conclusions of the lemma regarding $M_{i}, N_{i}$ and $\left.\sigma(H)\right|_{x=0}$ only depend on the normal operator of $\square$ in $x^{-2} \operatorname{Diff}_{\mathrm{e}}^{2}(M)$.

Now, the normal operator is determined by the restriction of $x^{-2} g$ to ${ }^{\mathrm{e}} T_{\partial X}^{*} X$, which, by (1.6), is $x^{-2}\left(d x^{2}+h(0, y, d y)\right)+k(0, y, z, 0,0, d z)$. The dual of $x^{-2} g$, as a symmetric edge 2-tensor, i.e. as a symmetric section of ${ }^{\mathrm{e}} T^{*} X \otimes{ }^{\mathrm{e}} T^{*} X$, is $x^{2}\left(\partial_{x}^{2}+H\left(0, y, \partial_{y}\right)\right)+K\left(0, y, z, 0,0, \partial_{z}\right)$ modulo sections vanishing at $\partial X$, with $H$, resp. $K$, the duals of $h$ and $k$. The standard expression for the Laplacian yields

$$
\Delta=D_{x}^{*} D_{x}+\sum_{i, j} D_{y_{i}}^{*} H^{i j}(y) D_{y_{j}}+\sum_{i, j} D_{z_{i}}^{*} x^{-2} K^{i j}(y, z) D_{z_{j}}+P,
$$

$P \in x^{-1} \operatorname{Diff}_{\mathrm{e}}^{2}(M)$. As $\operatorname{det} g=x^{2 f} a, a \in \mathcal{C}^{\infty}(X), a>0$,

$$
D_{y_{i}}^{*}-D_{y_{i}}=(\operatorname{det} g)^{-1 / 2} D_{y_{i}}(\operatorname{det} g)^{1 / 2}-D_{y_{i}} \in \mathcal{C}^{\infty}(X),
$$

and the lemma follows.

The $Q_{i}$ in (9.5) are homogeneous of degree -1 with respect to dilations in the $x$ factor in the trivialization. This leads to the term $4 a\left(\partial_{\underline{\xi}} a\right) \kappa_{i j}$ in $\sigma\left(L_{i j}\right)$ in the following commutator calculation; ultimately this is the dominant term. 
Lemma 9.6. With the notation of Lemma 9.5, if $A=A^{\dagger}=O p_{\operatorname{trv}}(a) \in$ $\Psi_{\mathrm{b}}^{m}(M)$ then

$$
\begin{gathered}
i\left[A^{*} A, \square\right]=\sum Q_{i}^{*} L_{i j} Q_{j}+\sum Q_{i}^{*} L_{i}^{\prime}+\sum L_{i}^{\prime \prime} Q_{i}+L_{0}+E \\
\text { with } L_{i j} \in \Psi_{\mathrm{b}}^{2 m-1}(M), L_{i}^{\prime}, L_{i}^{\prime \prime} \in \Psi_{\mathrm{b}}^{2 m}(M), L_{0} \in \Psi_{\mathrm{b}}^{2 m+1}(M), \\
E \in x^{-2} \operatorname{Diff}_{\mathrm{e}}^{2} \Psi_{\mathrm{b}}^{2 m-1}(M) \text { having } \mathrm{WF}_{\mathrm{b}}^{\prime}(E) \cap \dot{\Sigma}=\emptyset, \\
\sigma\left(L_{i j}\right)=4 a\left(\partial_{\underline{\xi}} a\right) \kappa_{i j}+a V_{i j} a, \sigma\left(L_{i}^{\prime}\right)=a V_{i}^{\prime} a, \\
\sigma\left(L_{i}^{\prime \prime}\right)=a V_{i}^{\prime \prime} a \text { and } \sigma\left(L_{0}\right)=a V_{0} a
\end{gathered}
$$

where the $V_{i j}, V_{i}^{\prime}, V_{i}^{\prime \prime}$ and $V_{0}$ are in the span, over homogeneous functions on ${ }^{\mathrm{b}} T^{*} M$ (independent of a), of vector fields on ${ }^{\mathrm{b}} T^{*}([0, \epsilon) \times Y$ ) that are tangent to $\{\underline{\xi}=c\}$ at $x=0$ for each $c$, and

$$
\left.V_{0}\right|_{x=0}=2 H_{h-\underline{\tau}^{2}} .
$$

We remark that while the factors in $x^{-k} \operatorname{Diff}_{\mathrm{e}}^{k} \Psi_{\mathrm{b}}^{m}(M)$ may be arbitrarily rearranged, according to Lemma 8.1 and Corollary 8.3, the principal symbols of the factors in the rearranged version may involve derivatives of the principal symbol of the original operators. This is due to the appearance of $Q_{0}=x^{-1}$ terms which are lower order than the $Q_{j}$ in the standard sense, but not in the sense of the grading of $x^{-k} \operatorname{Diff}_{\mathrm{e}}^{k} \Psi_{\mathrm{b}}^{m}(M)$. This is of no consequence if the terms being rearranged are a priori bounded. However, for positive commutator estimates such as those below, the principal symbols needs to be controlled, and the basic problem we face is that the differential of a non-zero function is never bounded by a multiple of the function near the boundary of its support, so in microlocalizing it is necessary to exclude such undesirable derivatives, hence the careful structuring of (9.8).

Proof. Using the decomposition of $\square$ in Lemma 9.5

$$
\begin{gathered}
(9.9)-i\left[A^{*} A, \square\right]=i \sum\left[A^{*} A, Q_{i}^{*}\right] \kappa_{i j} Q_{j}+i \sum Q_{i}^{*} \kappa_{i j}\left[A^{*} A, Q_{j}\right] \\
+i \sum\left[A^{*} A, Q_{i}^{*}\right] M_{i}+i \sum N_{i}\left[A^{*} A, Q_{i}\right] \\
+i \sum Q_{i}^{*}\left[A^{*} A, \kappa_{i j}\right] Q_{j}+i \sum Q_{i}^{*}\left[A^{*} A, M_{i}\right]+i \sum\left[A^{*} A, N_{i}\right] Q_{i}+i\left[A^{*} A, H\right] .
\end{gathered}
$$

The last four terms involve commutators of the form $i\left[A^{*} A, R\right]$, with $R \in$ $\operatorname{Diffl}_{\mathrm{b}}^{l}(M)$ and Lemma 8.4 shows that such terms are in $\Psi_{\mathrm{b}}^{2 m+l-1}(M)$, with principal symbol of the form $a V a$ with $V$ a homogeneous vector field on ${ }^{\mathrm{b}} T^{*} M$, tangent to $\xi=c$ at $x=0$. These terms make obvious contributions as required by $(9 . \overline{8})$, so it only remains to consider the terms involving commutators with the $Q_{i}$ and $Q_{i}^{*}$.

Consider first $Q_{1}=D_{x}$. Lemma 8.6 applies and shows that $i\left[A^{*} A, D_{x}\right]$ is of the form $B+C D_{x}$ with $B \in \Psi_{\mathrm{b}}^{2 m}(M)$, and $C \in \Psi_{\mathrm{b}}^{2 m-1}(M)$ where $\sigma(B)=-\partial_{x} a^{2}$ and $\sigma(C)=-\partial_{\underline{\xi}} a^{2}$. The term involving $B$ can then be absorbed in the $L_{i}^{\prime}, L_{i}^{\prime \prime}$ or $L_{0}$ terms, while $C$ enters into $L_{i j}$ (or $L_{i}^{\prime}, L_{i}^{\prime \prime}$, 
depending on which factor of $Q_{i}$ we were considering). Notice though that if $C$ enters into $L_{i}^{\prime}$, i.e. if we were considering $i\left[A^{*} A, Q_{i}^{*}\right] M_{i}^{\prime}$, the vanishing of the principal symbol of $M_{i}^{\prime}$ at $x=0$ shows that the principal symbol of $L_{i}^{\prime}$ is indeed given by $a V a$ with $V$ a vector field tangent to $\underline{\xi}=c$ at $x=0$, namely $x \partial_{\xi}$.

Next consider the terms $\left[A^{*} A, Q_{i}\right]$ with $Q_{i}=x^{-1} W_{i}$ for $i \neq 0$, to which Lemma 9.3 applies for all $W_{i}$. Since $F$ in Lemma 9.3 is in $\Psi_{\mathrm{b}}^{2 m-1}(M)$, for all $W_{i}$ but $Q_{0}$ (for which this commutator vanishes), we may regard $x^{-1} F$ as a term of the form $F^{\prime} Q_{0}, F^{\prime} \in \Psi_{\mathrm{b}}^{2 m-1}(M)$, thus giving terms as in the statement of the lemma.

So it remains to consider $i\left[A^{*} A, x^{-1}\right]$. This lies in $\Psi_{\mathrm{b}}^{2 m-1}(M)$, and by Lemma 8.4, it has principal symbol $-2 x^{-1} a \partial_{\underline{\xi}} a$. This completes the proof.

In $O_{\delta}=O \cap\{x<\delta\}$, with $|\underline{\hat{\zeta}}|$ arising from the metric on the fibers

$$
p_{0}=\sigma_{b}(\square)=\underline{\tau}^{2}-\left(\frac{\underline{\xi}^{2}}{x^{2}}+(1+O(x))|\underline{\eta}|_{h}^{2}+O(1) \underline{\eta \zeta}+\left(\frac{1}{x^{2}}+O(1)\right)|\underline{\zeta}|_{k}^{2}\right) \text {. }
$$

and for $\delta>0$ sufficiently small, $\underline{\hat{\xi}}^{2}+|\hat{\hat{\zeta}}|^{2}<c_{1} / 2$ in $\dot{\Sigma} \cap U_{0}, U_{0}={ }^{\mathrm{b}} T^{*} O_{\delta}$. The Hamilton vector field of $p_{0}$ in ${ }^{\mathrm{b}} \bar{T}^{*}(M)$ is then

$$
\begin{aligned}
H_{p_{0}} & =\underline{\tau} \partial_{t}-\frac{\underline{\xi}}{x} \partial_{x}-\frac{2\left(\underline{\xi}^{2}+|\underline{\zeta}|^{2}\right)}{x^{2}} \partial_{\underline{\xi}}-H_{y, \underline{\eta}}-\frac{1}{x^{2}} H_{z, \underline{\zeta}} \\
& +(O(x \underline{\eta})+O(\underline{\zeta})) \partial_{y}+\left(O\left(x \underline{\eta}^{2}\right)+O(\underline{\eta \zeta})+O\left(\underline{\zeta}^{2} / x^{2}\right)\right) \partial_{\underline{\eta}} \\
& +(O(\underline{\eta})+O(\underline{\zeta})) \partial_{z}+\left(O(\underline{\eta \zeta})+O\left(\underline{\zeta}^{2}\right)\right) \partial_{\underline{\zeta}} \\
& +\left(O\left(x \underline{\eta}^{2}\right)+O(x \underline{\eta \zeta})+O\left(\underline{\zeta}^{2} / x\right)\right) \partial_{\underline{\xi}} .
\end{aligned}
$$

where $H_{y, \eta}$ and $H_{z, \zeta}$ denote respectively the Hamilton vector fields of $h$ in $(y, \underline{\eta})$ (with $x$ as a parameter) and of $k$ in $(z, \underline{\zeta})$ (with $(x, y)$ as parameters). So

$$
\begin{aligned}
& -\underline{\hat{\xi}}=-\frac{\underline{\xi}}{|\underline{\tau}|} \text { satisfies } \\
& \quad H_{p_{0}}(-\underline{\hat{\xi}})=\frac{1}{|\underline{\tau}|}\left(\frac{\underline{\xi}^{2}}{x^{2}}+\frac{|\underline{\zeta}|^{2}}{x^{2}}+O\left(x \underline{\eta}^{2}\right)+O(x \underline{\eta \zeta})+O\left(\underline{\zeta}^{2} / x\right)\right) .
\end{aligned}
$$

The function $-\hat{\xi}$ is therefore a 'propagating variable' decreasing under the flow. The commutator argument used below makes essential use of this decrease.

Now we are ready to state and prove the diffractive propagation theorem in the hyperbolic region; the proof is similar to that of the corresponding result in [25]. 
Theorem 9.7. Let $u$ be an admissible solution of $\square u=0$ and suppose $U \subset \dot{\Sigma}$ is a neighborhood of $q_{0}=\left(t_{0}, \underline{\tau}_{0}, y_{0}, \underline{\eta}_{0}\right) \in \dot{\mathcal{H}}$ then

$$
U \cap\{-\underline{\hat{\xi}}<0\} \cap \mathrm{WF} u=\emptyset \Longrightarrow q_{0} \notin \mathrm{WF}_{\mathrm{b}, \tilde{\mathcal{D}}} u .
$$

Since $\mathrm{WF} u$ and $\mathrm{WF}_{\mathrm{b}, \tilde{\mathcal{D}}} u$ are the same away from $x=0$, and $\mathrm{WF}_{\mathrm{b}, \tilde{\mathcal{D}}}$ is a closed set, this result suffices to get propagation of wavefront set into and back out of the edge boundary along rays striking it transversely (i.e. arriving at points in $\dot{\mathcal{H}}$.)

Proof. Fix a small coordinate neighborhood $U_{0}$ of $q_{0}$. By Lemma 7.1 and the hypotheses, there exists $\delta>0$ such that

$\left\{q:|x(q)|^{2}+\left|y(q)-y_{0}\right|^{2}+\left|t(q)-t_{0}\right|^{2}+\left|\underline{\hat{\eta}}(q)-\underline{\hat{n}}_{0}\right|^{2}<\delta\right\} \cap\{-\underline{\hat{\xi}}<0\} \cap \mathrm{WF} u=\emptyset$

where $\underline{\hat{\eta}}=\frac{\eta}{|\underline{\tau}|}$. Set

$$
\begin{gathered}
\omega=x^{2}+\left(y-y_{0}\right)^{2}+\left(\underline{\hat{\eta}}-\underline{\hat{\eta}}_{0}\right)^{2}+\left(t-t_{0}\right)^{2} \text { and } \\
\phi=-\underline{\hat{\xi}}+\frac{1}{\beta^{2} \delta} \omega
\end{gathered}
$$

with the idea that at the edge, the propagation which we cannot control is in the 'fast variables' $(\underline{\xi}, \underline{\zeta})$ but we may localize in the remaining ('slow') variables by cutting off in $\omega$ and using a multiple of $-\underline{\hat{\xi}}$ to construct a positive commutator. Note that

$$
|\underline{\tau}|^{-1} H_{p_{0}} \omega=O\left(\sqrt{\omega}\left(\underline{\hat{\xi}}^{2} / x^{2}+\underline{\hat{\zeta}}^{2} / x^{2}+1\right)\right) .
$$

Next we select some cut-off functions. First choose $\chi_{0} \in \mathcal{C}^{\infty}(\mathbb{R})$ with support in $[0, \infty)$ and $\chi_{0}(s)=\exp (-1 / s)$ for $s>0$. Thus, $\chi_{0}^{\prime}(s)=s^{-2} \chi_{0}(s)$. Take $\chi_{1} \in \mathcal{C}^{\infty}(\mathbb{R})$ to have support in $[0, \infty)$, to be equal to 1 on $[1, \infty)$ and to have $\chi_{1}^{\prime} \geq 0$ with $\chi_{1}^{\prime} \in \mathcal{C}_{c}^{\infty}((0,1))$. Finally, let $\chi_{2} \in \mathcal{C}_{c}^{\infty}(\mathbb{R})$ be supported in $\left[-2 c_{1}, 2 c_{1}\right]$ and be identically equal to 1 on $\left[-c_{1}, c_{1}\right]$. Here we will take $c_{1}$ to be such that

$$
\underline{\hat{\xi}}^{2}+|\underline{\hat{\zeta}}|^{2}<c_{1} / 2 \text { in } \dot{\Sigma} \cap U_{0}
$$

where $|\underline{\hat{\zeta}}|$ is the metric length of fiber covectors with respect to some fiber metric. Thus, $\chi_{2}\left(\underline{\hat{\xi}}^{2}+|\underline{\hat{\zeta}}|^{2}\right)$ is a cut-off such that $d \chi_{2}\left(\underline{\hat{\xi}}^{2}+|\underline{\hat{\zeta}}|^{2}\right)$ is supported in $\underline{\hat{\xi}}^{2}+|\hat{\hat{\zeta}}|^{2} \in\left[c_{1}, 2 c_{1}\right]$ hence outside $\dot{\Sigma}$. Such a factor microlocalizes near the characteristic set but effectively commutes with $\square$. We shall further insist that all cut-offs and their derivatives have (up to sign) smooth square roots.

Now consider the test symbol

$$
a=\chi_{0}(1-\phi / \delta) \chi_{1}(-\underline{\hat{\xi}} / \delta+1) \chi_{2}\left(\underline{\hat{\xi}}^{2}+|\underline{\hat{\zeta}}|^{2}\right) .
$$


We proceed to show that

(9.18)

for given $\beta>0$, if $\delta>0$ is sufficiently small then $a \in \mathcal{C}^{\infty}\left({ }^{\mathrm{b}} T^{*} M \backslash 0\right)$

is a basic symbol with support in any preassigned

$$
\text { conic neighborhood of } q_{0} \in{ }^{\mathrm{b}} \dot{S}^{*} M \text {. }
$$

To see this, observe that from the choices made for the cut-offs, $\phi \leq \delta$ and $\underline{\hat{\xi}} \leq \delta$ on the supports. Since $\omega \geq 0$ in $(9.14),-\underline{\hat{\xi}} \leq \delta$ so

$$
|\underline{\hat{\xi}}| \leq \delta \Longrightarrow \omega \leq \beta^{2} \delta(\phi+\underline{\hat{\xi}}) \leq \beta^{2} \delta(\delta+\underline{\hat{\xi}}) \leq 2 \delta^{2} \beta^{2} .
$$

In view of (9.14) and (9.13), this shows that for any $\beta>0, a \in \mathcal{C}^{\infty}\left({ }^{\mathrm{b}} T^{*} M \backslash\right.$ 0 ) is supported in a given conic neighborhood of $q_{0}$, provided $\delta>0$ is sufficiently small. Since the only dependence on the fiber in (9.17) comes through the metric, as $|\hat{\hat{\zeta}}|^{2}$, and $\chi_{2}$ is constant near $0, a$ is constant on the fibers near $\underline{\hat{\xi}}^{2}+|\hat{\hat{\zeta}}|^{2}=0$, which is to say it is basic; this proves (9.18).

Now, we may take $A=\operatorname{Op}_{\text {trv }}\left(a_{0}\right)$ using Definition 9.1 with $a_{0}$ from (9.17). To extract some positivity from the commutator of $A^{*} A$ with $\square$ using Lemma 9.6 we need to show that the term $4 a \partial_{\xi} a \kappa_{i j}$ in the symbol of $\sigma\left(L_{i j}\right)$ dominates the others. To do this it is enough to show that for a homogeneous vector field $V$, tangent to $\underline{\xi}=0, V a$ can be estimated by $\partial_{\xi} a$, if $\beta, \delta>0$ are chosen appropriately.

We shall rewrite the identity (9.8) for this particular choice of $A$, using (9.7). Choose a basic operator $\tilde{B} \in \Psi_{\mathrm{b}}^{1 / 2}(M)$ with

$$
\tilde{b}=\sigma_{\mathrm{b}, 1 / 2}(\tilde{B})=|\underline{\tau}|^{1 / 2} \delta^{-1 / 2}\left(\chi_{0} \chi_{0}^{\prime}\right)^{1 / 2} \chi_{1} \chi_{2} \in \mathcal{C}^{\infty}\left({ }^{\mathrm{b}} T^{*} M \backslash 0\right)
$$

where the regularity uses the properties of the cut-offs, in particular that they and their derivatives are squares. Thus $\tilde{b}^{2}$ occurs as a factor in any first derivative of $a$ in which the derivatives strike the $\chi_{0}$ term. (Note that other derivatives are acceptable either because they are supported away from $\dot{\Sigma}$ or because they are supported in the region where we have assume regularity.) Also, choose $C \in \Psi_{\mathrm{b}}^{0}(M)$ with symbol

$$
\sigma_{\mathrm{b}, 0}(C)=|\underline{\tau}|^{-1}\left(2 \underline{\tau}^{2}-2|\underline{\eta}|_{y}^{2}\right)^{1 / 2} \psi
$$

where $\psi \in S^{0}\left({ }^{\mathrm{b}} T^{*} M\right)$ is identically 1 on $U$ considered as a subset of ${ }^{\mathrm{b}} T^{*} M$. Then, recalling that the $Q_{i}$ are given in (9.5) we proceed to show that

$$
\begin{gathered}
i\left[A^{*} A, \square\right]=\sum Q_{i}^{*} L_{i j} Q_{j}+\sum Q_{i}^{*} L_{i}^{\prime}+\sum L_{i}^{\prime \prime} Q_{i}+L_{0}+E \\
=R^{\prime} \square+\tilde{B}^{*}\left(C^{*} C+R_{0}+\sum_{i} Q_{i} R_{i}+\sum_{i j} Q_{i} R_{i j} Q_{j}\right) \tilde{B}+R^{\prime \prime}+E^{\prime}+E^{\prime \prime}
\end{gathered}
$$


where all factors have wavefront set near $q_{0}$,

$$
\begin{gathered}
R_{0} \in \Psi_{\mathrm{b}}^{0}(M), R_{i} \in \Psi_{\mathrm{b}}^{-1}(M), R_{i j} \in \Psi_{\mathrm{b}}^{-2}(M), \\
R^{\prime} \in \Psi_{\mathrm{b}}^{-1}(M), R^{\prime \prime} \in x^{-2} \operatorname{Diff}_{\mathrm{e}}^{2} \Psi_{\mathrm{b}}^{-2}(M), \\
E^{\prime}, E^{\prime \prime} \in x^{-2} \operatorname{Diff}_{\mathrm{e}}^{2} \Psi_{\mathrm{b}}^{-1}(M),
\end{gathered}
$$

have symbols

$$
\begin{gathered}
r_{0}=\sigma_{\mathrm{b}, 0}\left(R_{0}\right), r_{i}=\sigma_{\mathrm{b},-1}\left(R_{i}\right), r_{i j} \in \sigma_{\mathrm{b},-2}\left(R_{i j}\right) \\
\text { satisfying } \operatorname{supp}\left(r_{j}\right) \subset\left\{\omega \leq 9 \delta^{2} \beta^{2}\right\},\left|r_{0}\right| \leq C_{2}\left(1+\frac{1}{\beta^{2} \delta}\right) \omega^{1 / 2}, \\
\left|\underline{\tau} r_{i}\right| \leq C_{2}\left(1+\frac{1}{\beta^{2} \delta}\right) \omega^{1 / 2},\left|\underline{\tau}^{2} r_{i j}\right| \leq C_{2}\left(1+\frac{1}{\beta^{2} \delta}\right) \omega^{1 / 2}, \\
\left|r_{0}\right| \leq 3 C_{2}\left(\delta \beta+\beta^{-1}\right),\left|\underline{\tau} r_{i}\right| \leq 3 C_{2}\left(\delta \beta+\beta^{-1}\right), \\
\left|\underline{\tau}^{2} r_{i j}\right| \leq 3 C_{2}\left(\delta \beta+\beta^{-1}\right)
\end{gathered}
$$

and

$$
\mathrm{WF}_{\mathrm{b}}^{\prime}\left(E^{\prime}\right) \subset \underline{\hat{\xi}}^{-1}((0, \infty)) \cap U, \mathrm{WF}_{\mathrm{b}}^{\prime}\left(E^{\prime \prime}\right) \cap \dot{\Sigma}=\emptyset .
$$

The first equality in (9.22) is just (9.8). The operators $L_{i}^{\prime}$ have symbols as described in (9.8) and in particular these are smooth multiples of $\tilde{b}^{2}$ plus noncharacteristic terms and terms supported in $\hat{\xi}^{-1}((0, \infty))$ so we may write $L_{i}^{\prime}=\tilde{B}^{*} R_{i} \tilde{B}+R_{i}^{\prime}+E^{\prime}+E^{\prime \prime}$ where $R_{i}^{\prime} \in \Psi_{\mathrm{b}}^{-2}(M)$ and the $E^{\prime}, E^{\prime \prime}$ terms satisfy (9.25); henceforth, the meaning of the terms $E^{\prime}$ and $E^{\prime \prime}$ (and corresponding symbols $\left.e^{\prime}, e^{\prime \prime}\right)$ will change from line to line, but all will satisfy (9.25). Thus

$$
\sum_{i} Q_{i}^{*} L_{i}^{\prime}=\tilde{B}^{*} \sum_{i} Q_{i}^{*} R_{i} \tilde{B}^{*}+\sum_{i}\left[Q_{i}^{*}, \tilde{B}^{*}\right] R_{i} \tilde{B}+\sum_{i} Q_{i}^{*} R_{i}^{\prime}
$$

Since $\tilde{B}$ is basic, the commutator term may be absorbed into $E^{\prime \prime}$. The final term may be absorbed into $R^{\prime \prime}$ in $(9.22)$. The $L_{i}^{\prime \prime} Q_{i}$ terms are similar, with a final commutation of $Q_{i}$ to the left. Similarly, $L_{0}$ gives rise to a term involving $R_{0}$ with additional contributions to $R^{\prime \prime}$ and $E^{\prime \prime}$.

Now consider the first term in which the $L_{i j}$ appear. The definitions above give

$$
\begin{aligned}
& 4 a \partial_{\underline{\xi}} a=-\frac{4}{|\underline{\tau}|^{2}} \tilde{b}^{2}+r_{i j}+e^{\prime}+e^{\prime \prime} \\
& \quad \Longrightarrow L_{i j}=B^{*}\left(T^{*} \kappa_{i j} T+R_{i j}\right) B+L_{i j}^{\prime}+E^{\prime}+E^{\prime \prime}, L_{i j}^{\prime} \in \Psi_{\mathrm{b}}^{-2}(M)
\end{aligned}
$$


where $T \in \Psi_{\mathrm{b}}^{-1}(M)$ is elliptic. The sum involving the $L_{i j}$ in (9.22) can therefore be written, using (9.7)

$$
\begin{aligned}
& \sum_{i} Q_{i}^{*} L_{i j} Q_{j}=\tilde{R}^{\prime} \square \\
+ & B^{*}\left(-H-\sum_{i} Q_{i}^{*} M_{i}-\sum_{i} N_{i} Q_{i}+\sum_{i j} Q_{i} R_{i j} Q_{j}\right) \tilde{B}+\sum Q_{i}^{*} L_{i j}^{\prime} Q_{j}+E^{\prime}+E^{\prime \prime}
\end{aligned}
$$

where the $E^{\prime}, E^{\prime \prime}$ terms now involve commutators of the $Q_{i}$ or $Q_{i}^{*}$ and $\tilde{B}$ or $\tilde{B}^{*}$, as well as commutators involving $T, T^{*}$. This leads to the final line in (9.22).

By Lemma 9.6, the symbol $r_{i j}$ can be written as $\chi_{0} V \chi_{0}$ times $\chi_{1}$ and $\chi_{2}$ factors, with $V$ tangent to $\underline{\xi}=c$ at $x=0$. (We have absorbed $\chi_{1}^{\prime}, \chi_{2}^{\prime}$ terms in $E^{\prime}, E^{\prime \prime}$.) As we can estimate $V \omega$ by a multiple of $\sqrt{\omega}$ and $V \xi$ by a multiple of $x$ and hence of $\sqrt{\omega}$, we obtain the desired symbol estimates. The estimates on $r_{i}$ and $r_{0}$ proceed analogously.

Having calculated the commutator in (9.22), we proceed to estimate the 'error terms' $R_{0}, R_{i}, R_{i j}$ as operators. We start with $R_{0}$. As follows from the standard square root construction to prove the $L^{2}$ boundedness of pseudodifferential operators, there exists $R_{0}^{\prime} \in \Psi_{\mathrm{b}}^{-1}(M)$ such that

$$
\left\|R_{0} v\right\| \leq 2 \sup \left|r_{0}\right|\|v\|+\left\|R_{0}^{\prime} v\right\|
$$

for all $v \in L^{2}(M, d g)$. Here $\|\cdot\|$ is the $L^{2}(M, d g)$-norm, as usual. Thus, we can estimate, for any $\gamma>0$,

$$
\begin{aligned}
\left|\left\langle R_{0} v, v\right\rangle\right| \leq\left\|R_{0} v\right\| \| & v\left\|\leq 2 \sup \left|r_{0}\right|\right\| v\left\|^{2}+\right\| R_{0}^{\prime} v\|\| v \| \\
& \leq 6 C_{2}\left(\delta \beta+\beta^{-1}\right)\|v\|^{2}+\gamma^{-1}\left\|R_{0}^{\prime} v\right\|^{2}+\gamma\|v\|^{2} .
\end{aligned}
$$

Now we turn to $R_{i}$. Let $T_{-1} \in \Psi_{\mathrm{b}}^{-1}(M)$ be elliptic (we will use this to keep track of orders), and $T_{1} \in \Psi_{\mathrm{b}}^{1}(M)$ a parametrix, so $T_{1} T_{-1}=\mathrm{Id}+F$, $F \in \Psi_{\mathrm{b}}^{-\infty}(M)$. Then there exist $R_{i}^{\prime} \in \Psi_{\mathrm{b}}^{-1}(M)$ such that

$$
\begin{aligned}
\left\|R_{i} w\right\|=\left\|R_{i}\left(T_{1} T_{-1}-F\right) w\right\| & \leq\left\|\left(R_{i} T_{1}\right)\left(T_{-1} w\right)\right\|+\left\|R_{i} F w\right\| \\
& \leq 6 C_{2}\left(\delta \beta+\beta^{-1}\right)\left\|T_{-1} w\right\|+\left\|R_{i}^{\prime} T_{-1} w\right\|+\left\|R_{i} F w\right\|
\end{aligned}
$$

for all $w$ with $T_{-1} w \in L^{2}(M, d g)$. Similarly, there exist $R_{i j}^{\prime} \in \Psi_{\mathrm{b}}^{-1}(M)$ such that

(9.29) $\left\|\left(T_{1}\right)^{*} R_{i j} w\right\| \leq 6 C_{2}\left(\delta \beta+\beta^{-1}\right)\left\|T_{-1} w\right\|+\left\|R_{i j}^{\prime} T_{-1} w\right\|+\left\|\left(T_{1}\right)^{*} R_{i j} F w\right\|$

for all $w$ with $T_{-1} w \in L^{2}(M, d g)$. Thus,

$$
\begin{aligned}
\left|\left\langle R_{i} Q_{i} v, v\right\rangle\right| \leq 6 C_{2}( & \left.\delta \beta+\beta^{-1}\right)\left\|T_{-1} Q_{i} v\right\|\|v\| \\
& +2 \gamma\|v\|^{2}+\gamma^{-1}\left\|R_{i}^{\prime} T_{-1} Q_{i} v\right\|^{2}+\gamma^{-1}\left\|F_{i} Q_{i} v\right\|^{2},
\end{aligned}
$$


and, writing $Q_{j} v=T_{1} T_{-1} Q_{j} v-F Q_{j} v$ in the right factor, and taking the adjoint of $T_{1},(9.29)$ gives

$$
\begin{aligned}
& \text { (9.31) }\left|\left\langle R_{i j} Q_{i} v, Q_{j} v\right\rangle\right| \leq 6 C_{2}\left(\delta \beta+\beta^{-1}\right)\left\|T_{-1} Q_{i} v\right\|\left\|T_{-1} Q_{j} v\right\| \\
& +2 \gamma\left\|T_{-1} Q_{j} v\right\|^{2}+\gamma^{-1}\left\|R_{i j}^{\prime} T_{-1} Q_{i} v\right\|^{2}+\gamma^{-1}\left\|F_{i j} Q_{i} v\right\|^{2}+\left\|R_{i j} Q_{i} v\right\|\left\|F Q_{j} v\right\|,
\end{aligned}
$$

with $F_{i}, F_{i j} \in \Psi_{\mathrm{b}}^{-\infty}(M)$.

Finally we turn to the positive commutator argument itself. Let $s<$ $\sup \left\{s^{\prime}: q_{0} \notin \mathrm{WF}_{\mathrm{b}, \tilde{\mathcal{D}}} u\right\}$; hence, without loss of generality, we have $\mathrm{WF}_{\mathrm{b}, \tilde{\mathcal{D}}}^{s} u \cap$ $U=\emptyset$ where we shrink $U$ as necessary. We will prove that $q_{0} \notin \mathrm{WF}_{\mathrm{b}, \tilde{\mathcal{D}}}^{s+1 / 2} u$, a contradiction unless $s=+\infty$.

Let $\Lambda_{r}$ be a quantization of

$$
|\underline{\tau}|^{s}\left(1+r|\underline{\tau}|^{2}\right)^{-s / 2}, \quad r \in[0,1)
$$

and set $A_{r}=A \Lambda_{r} \in \Psi_{\mathrm{b}}^{0}(M)$ for $r>0$. Then $A_{r}$ is uniformly bounded in $\Psi_{\mathrm{b} \infty}^{s}(M)$, and we may further arrange that $\left[\square, \Lambda_{r}\right]=0$.

By (9.22),

$$
\begin{aligned}
\left\langle i\left[A_{r}^{*} A_{r}, \square\right] u, u\right\rangle=\| & C \tilde{B} \Lambda_{r} u \|^{2}+\left\langle R^{\prime} \square \Lambda_{r} u, \Lambda_{r} u\right\rangle+\left\langle R_{0} \tilde{B} \Lambda_{r} u, \tilde{B} \Lambda_{r} u\right\rangle \\
& +\sum\left\langle R_{i} Q_{i} \tilde{B} \Lambda_{r} u, \tilde{B} \Lambda_{r} u\right\rangle+\sum\left\langle R_{i j} Q_{i} \tilde{B} \Lambda_{r} u, Q_{j} \tilde{B} \Lambda_{r} u\right\rangle \\
& +\left\langle R^{\prime \prime} \Lambda_{r} u, \Lambda_{r} u\right\rangle+\left\langle\left(E+E^{\prime}\right) \Lambda_{r} u, \Lambda_{r} u\right\rangle .
\end{aligned}
$$

On the other hand, as $A_{r} \in \Psi_{\mathrm{b}}^{0}(M)$ for $r>0$ and $u \in \tilde{\mathcal{D}}, A_{r}^{*} A_{r} u \in \tilde{\mathcal{D}}$, and the pairing in the following computation is well-defined:

$$
\begin{aligned}
\left\langle\left[A_{r}^{*} A_{r}, \square\right] u, u\right\rangle=\left\langle A_{r}^{*} A_{r} \square u, u\right\rangle & -\left\langle\square A_{r}^{*} A_{r} u, u\right\rangle \\
& =\left\langle A_{r} \square u, A_{r} u\right\rangle-\left\langle A_{r} u, A_{r} \square u\right\rangle=0 .
\end{aligned}
$$

The first term on the right in (9.33) is thus bounded by the sum of the absolute values of the others. The second term on the right in (9.33) vanishes, to the third we can apply (9.28), to the fourth (9.30) and (9.31) applies to the fifth. The penultimate term is bounded uniformly as $r \downarrow 0$ by the hypothesis on $s$, i.e. the assumed regularity of $u$. The last term is also uniformly bounded, the $E$ part for the same reason and the $E^{\prime}$ term by elliptic regularity. Similarly, in applying (9.28) - (9.31) all the terms but the first two terms on the right in each are also uniformly bounded as $r \downarrow 0$ by the assumed property of $\mathrm{WF}_{\mathrm{b}, \tilde{\mathcal{D}}}^{s} u$. So, with a constant $C^{\prime}(\gamma)$ independent of $r$ 
and for some $C_{3}>0$ depending only on the geometry

$$
\begin{aligned}
& \left\|C \tilde{B} \Lambda_{r} u\right\|^{2} \leq C^{\prime}(\gamma) \\
& +\left(6 C_{2}\left(\delta \beta+\beta^{-1}\right)+C_{3} \gamma\right)\left(\left\|\tilde{B} \Lambda_{r} u\right\|^{2}+\sum_{i}\left\|T_{-1} Q_{i} \tilde{B} \Lambda_{r} u\right\|^{2}\right) \\
& \quad+6 C_{2}\left(\delta \beta+\beta^{-1}\right)\left\|\tilde{B} \Lambda_{r} u\right\| \sum_{i}\left\|T_{-1} Q_{i} \tilde{B} \Lambda_{r} u\right\|+2 \gamma\left\|\tilde{B} \Lambda_{r} u\right\|^{2} .
\end{aligned}
$$

The remaining terms on the right may be estimated as follows: writing $T_{-1} Q_{i}=Q_{i} T_{i}^{\prime}+x^{-1} T_{i}^{\prime \prime}$ for some $T_{i}^{\prime}, T_{i}^{\prime \prime} \in \Psi_{\mathrm{b}}^{-1}(M)\left(\right.$ recall that $\left.x^{-1}=Q_{0}\right)$, and using Lemma 8.8 where necessary, we may choose our constants so as to absorb the highest order terms into the left-hand-side (using ellipticity of $C$ on $\mathrm{WF}^{\prime}(\tilde{B})$ and microlocal elliptic regularity). In particular, then, we may pick $\beta$ sufficiently large, and $\gamma=\gamma_{0}>0, \delta_{0}>0$, so small that for all $\delta<\delta_{0}$

$$
C_{4}\left\|\tilde{B} \Lambda_{r} u\right\|^{2} \leq C_{5}+C_{6}\left\|d_{M} T_{-1}^{2} \tilde{B} \Lambda_{r} u\right\|^{2},
$$

with $C_{4}>0$. Letting $r \rightarrow 0$ now keeps the right hand side bounded, proving that $\left\|\tilde{B} \Lambda_{r} u\right\|$ is uniformly bounded as $r \rightarrow 0$, hence $\tilde{B} \Lambda_{0} u \in L^{2}(M, d g)$ (cf. the proof of Proposition 8.10). In view of Lemma 8.8 this proves that $q_{0} \notin \mathrm{WF}_{\mathrm{b}, \tilde{\mathcal{D}}}^{s+1 / 2}(u)$, and hence proves the first statement of the proposition.

Finally we need check that the neighborhoods of $q_{0}$ which are disjoint from $\mathrm{WF}_{\mathrm{b}, \tilde{\mathcal{D}}}^{s}(u)$ do not shrink to $\left\{q_{0}\right\}$ as $s \rightarrow \infty$. This argument is parallel to the last paragraph of the proof of [9, Proposition 24.5.1]. In each iterative step it is only necessary to shrink the elliptic set of $\tilde{B}_{s}$ by an arbitrarily small amount, which allows us to conclude that $q_{0}$ has a neighborhood $U^{\prime}$ such that $\mathrm{WF}_{\mathrm{b}, \tilde{\mathcal{D}}}^{s}(u) \cap U^{\prime}=\emptyset$ for all $s$. This proves that $q_{0} \notin \mathrm{WF}_{\mathrm{b}, \tilde{\mathcal{D}}}^{\infty}(u)$.

We note that in the proof above the ability to control $Q_{0} u$ terms in terms of the $\|u\|_{\tilde{\mathcal{D}}}$ was crucial, and it is this aspect of the proof that breaks down in fiber dimension 1 . The necessary modifications to the proof in this case are discussed in the following section.

To carry through similar estimates in the glancing region we need the following technical lemma. In essence this shows that, applied to solutions of $\square u=0$ near $\dot{\mathcal{G}}, D_{x}$ and $x^{-1} D_{z_{j}}$ are not merely bounded by $D_{t}$ but small compared to it. Such an estimate is natural since

$$
\left.x^{2} p_{0}\right|_{x=0}=x^{2} \tau^{2} \hat{p}_{0}=\tau^{2}-\xi^{2}-|\eta|_{h}^{2}-|\zeta|_{k}^{2}
$$

gives

$$
\underline{\hat{\xi}}^{2}+|\hat{\hat{\zeta}}|_{k}^{2} \leq C\left(x^{2}|\hat{p}|+|x|+\left.|1-| \underline{\hat{\eta}}\right|_{h} ^{2} \mid\right),
$$

and $1-|\hat{\eta}|_{h}^{2}$ vanishes at $\dot{\mathcal{G}}$, so the right hand side is small near $\dot{\mathcal{G}}$. In the remainder of this section, a $\delta$-neighborhood will refer to a $\delta$-neighborhood with respect to the metric distance associated to any Riemannian metric on the manifold ${ }^{\mathrm{b}} S^{*} M$. The notation $\|u\|_{\tilde{\mathcal{D}}_{\text {loc }}}$ and $\|\square u\|_{\tilde{\mathcal{D}}_{\text {loc }}^{\prime}}^{2}$ is fixed in Remark 8.7. 
Lemma 9.8. Suppose $u \in \tilde{\mathcal{D}}_{\text {loc }}$ and that $K \subset{ }^{\mathrm{b}} S^{*} M$ is compact with

$$
K \subset \mathcal{G} \backslash \mathrm{WF}_{\mathrm{b}, \tilde{\mathcal{D}}^{\prime}}^{s+1 / 2}(\square u)
$$

then there exist $\delta_{0}>0$ and $C_{0}>0$ such that if $\mathcal{A}=\left\{A_{r}: r \in(0,1]\right\}$ is a bounded and basic family in $\Psi_{\mathrm{b} \infty}^{s}(M)$ with $\mathrm{WF}_{\mathrm{b}}^{\prime}(\mathcal{A}) \subset U \subset{ }^{\mathrm{b}} S^{*} M$, a $\delta$-neighborhood of $K, 0<\delta<\delta_{0}$, and with $A_{r} \in \Psi_{\mathrm{b}}^{s-1}(M)$ for $r \in(0,1]$ then for some

$$
G \in \Psi_{\mathrm{b}}^{s-1 / 2}(M), \tilde{G} \in \Psi_{\mathrm{b}}^{s+1 / 2}(M) \text { with } \operatorname{WF}_{\mathrm{b}}^{\prime}(G), \mathrm{WF}_{\mathrm{b}}^{\prime}(\tilde{G}) \subset U
$$

and $\tilde{C}_{0}=\tilde{C}_{0}(\delta)>0$

$$
\begin{aligned}
& \left\|D_{x} A_{r} u\right\|^{2}+\sum_{i}\left\|x^{-1} d_{Z} A_{r} u\right\|_{k}^{2} \\
\leq & C_{0} \delta\left\|D_{t} A_{r} u\right\|^{2}+\tilde{C}_{0}\left(\|u\|_{\tilde{\mathcal{D}}_{l o c}}^{2}+\|G u\|_{\tilde{\mathcal{D}}^{2}}^{2}+\|\square u\|_{\tilde{\mathcal{D}}_{l o c}^{\prime}}^{2}+\|\tilde{G} \square u\|_{\tilde{\mathcal{D}}^{\prime}}^{2}\right) \forall r>0 .
\end{aligned}
$$

Remark 9.9. As $K$ is compact, this is a local result in the extended base, $[0, \epsilon)_{x} \times Y$. In particular, we may assume that $K$ is a subset of ${ }^{\mathrm{b}} S^{*} M$ over a suitable local coordinate patch in the extended base. Moreover, we may assume that $\delta_{0}>0$ is so small that $D_{t}$ is elliptic on $U$.

Proof. The proof is again very similar to [25]. By Lemma 8.8 we already know that

$$
\begin{aligned}
\left\|d_{X} A_{r} u\right\|^{2} \leq & \left\|D_{t} A_{r} u\right\|^{2} \\
& +C_{0}^{\prime}\left(\|u\|_{\tilde{\mathcal{D}}_{\text {loc }}}^{2}+\|G u\|_{\tilde{\mathcal{D}}^{\prime}}^{2}+\|\square u\|_{\tilde{\mathcal{D}}_{\text {loc }}^{\prime}}^{2}+\|\tilde{G} \square u\|_{\tilde{\mathcal{D}}^{\prime}}^{2}\right) .
\end{aligned}
$$

for some $C_{0}^{\prime}>0$ and for some $G, \tilde{G}$ as in the statement of the lemma. Thus, we only need to show that if we replace the left hand side by $\left\|D_{x} A_{r} u\right\|^{2}+$ $\left\|x^{-1} d_{Z} A_{r} u\right\|_{k}^{2}$ (i.e. we drop the tangential derivatives, at least roughly speaking), the constant in front of $\left\|D_{t} A_{r} u\right\|^{2}$ can be made small.

A simple argument as in [25], amounting to moving the tangential derivatives to the right side and freezing the coefficients at $x=0$ (cf. also the proof of Proposition 8.10), reduces the problem to showing that

$$
\begin{aligned}
& \left|\int_{M}\left(\left(D_{t}^{2}-\sum h_{i j}(0, y) D_{y_{i}} D_{y_{j}}\right) A_{r} u \overline{A_{r} u}\right) d g\right| \\
& \quad \leq C_{2} \delta\left\|D_{t} A_{r} u\right\|^{2}+\tilde{C}_{2}(\delta)\left(\|u\|_{\tilde{\mathcal{D}}_{\text {loc }}}^{2}+\|G u\|_{\tilde{\mathcal{D}}}^{2}\right),
\end{aligned}
$$

which we proceed to do.

The key point is that the symbol of $D_{t}^{2}-\sum h_{i j}(0, y) D_{y_{i}} D_{y_{j}}$ is small on $\mathrm{WF}_{\mathrm{b}}^{\prime}(\mathcal{A})$; more precisely, it is of size $\delta$. It is convenient to microlocalize, i.e. replace this tangential part of the wave operator by an operator $F$ microsupported near $\mathcal{G}$. So let $\psi \in \mathcal{C}^{\infty}\left({ }^{\mathrm{b}} S^{*} M\right)(\psi$ can thus be identified with a homogeneous degree zero function on $\left.{ }^{\mathrm{b}} T^{*} M \backslash 0\right)$ with $\psi \equiv 1$ near $\operatorname{WF}_{\mathrm{b}}^{\prime}(\mathcal{A})$, 
$\operatorname{supp} \psi \subset U,|\psi| \leq 1$, and let $F \in \Psi_{\mathrm{b}}^{0}(M)$ be such that

$$
\begin{array}{r}
\mathrm{WF}_{\mathrm{b}}^{\prime}(F) \subset U, \mathrm{WF}_{\mathrm{b}}^{\prime}\left(D_{t} F D_{t}-\left(D_{t}^{2}-\sum h_{i j} D_{y_{i}} D_{y_{j}}\right)\right) \cap \mathrm{WF}_{\mathrm{b}}^{\prime}(\mathcal{A})=\emptyset \\
f=\sigma_{\mathrm{b}, 0}(F)=\psi\left(1-\sum h_{i j} \underline{\hat{\eta}}_{i} \hat{\underline{\eta}}_{j}\right),
\end{array}
$$

where such $\psi$ and $F$ exist, since $D_{t}$ is elliptic on $\operatorname{WF}_{\mathrm{b}}^{\prime}(\mathcal{A})$. Now,

$$
\left|\int_{M}\left(\left(D_{t} F D_{t}-\left(D_{t}^{2}-\sum h_{i j}(0, y) D_{y_{i}} D_{y_{j}}\right)\right) A_{r} u \overline{A_{r} u}\right)\right| \leq C_{2}^{\prime}\|u\|_{\tilde{\mathcal{D}}_{\text {loc }}}^{2}
$$

since $\left(D_{t} F D_{t}-\left(D_{t}^{2}-\sum h_{i j} D_{y_{i}} D_{y_{j}}\right)\right) A_{r}$ is uniformly bounded in $\Psi_{\mathrm{b}}^{-\infty}(M)$, by the first line of (9.38). Moreover, $1-\sum h_{i j} \underline{\hat{\eta}}_{i} \hat{\underline{\eta}}_{j}$ is a $\mathcal{C}^{\infty}$ function on a neighborhood of $K$ in ${ }^{\mathrm{b}} S^{*} M$ which vanishes at $\mathcal{G}$, so $\left|1-\sum h_{i j} \underline{\hat{\zeta}}_{i} \underline{\hat{\zeta}}_{j}\right|<C_{3} \delta$ on a $\delta$-neighborhood of $K$, and hence

$$
\sup |f| \leq C_{3} \delta \text {. }
$$

Since there exists $F^{\prime} \in \Psi_{\mathrm{b}}^{-1}(M)$ with $\mathrm{WF}_{\mathrm{b}}^{\prime}\left(F^{\prime}\right) \subset U$ satisfying

$$
\|F v\| \leq 2 \sup |f|\|v\|+\left\|F^{\prime} v\right\|
$$

for all $v \in L^{2}(M, d g)$, we deduce that $\|F v\| \leq 2 C_{3} \delta\|v\|+\left\|F^{\prime} v\right\|$ for all $v \in L^{2}(M, d g)$. Applying this with $v=D_{t} A_{r} u$, and estimating $\left\|F^{\prime} v\right\|$ using Lemma $6.8,(9.37)$ follows, which in turn completes the proof of the lemma.

For propagation at the glancing points it is convenient to introduce notation for the Hamilton vector field of the 'tangential part' of the wave operator. Thus, we let

$$
W^{b}=\partial_{t}-H_{\hat{h}}, \hat{h}(y, \underline{\eta})=|\underline{\tau}|^{-1}|\underline{\eta}|_{h}^{2},
$$

so $W^{b}$ is homogeneous of degree zero, hence can be regarded as a vector field on the cosphere bundle. This vector field is well-defined at $\mathcal{G}$ as a vector field tangent to $\mathcal{G}$. Away from $\mathcal{G}$ it depends on choices, but as pointed out in a remark below, these choices do not affect the statement of the following proposition. Moreover, this vector field also makes sense on $\dot{\mathcal{G}}$, which is a smooth manifold.

It is also useful to extend $\dot{\pi}$ to a neighborhood of $\partial M$ as projection to $\partial M$ followed by $\dot{\pi}$ :

$$
\dot{\pi}^{\mathrm{e}}(x, t, y, z, \hat{\xi}, 1, \hat{\eta}, \hat{\zeta})=(t, y, 1, \hat{\eta}) .
$$

Proposition 9.10. Let $u \in \tilde{\mathcal{D}}_{\text {loc }}$ and suppose

$$
K \subset{ }^{\mathrm{b}} S^{*} M \text { is compact with } K \subset \dot{\mathcal{G}} \backslash \mathrm{WF}_{\mathrm{b}, \tilde{\mathcal{D}}^{\prime}}^{\infty}(\square u) .
$$

Then there exist constants $C_{0}>0, \delta_{0}>0$ such that if $q_{0}=\left(t_{0}, y_{0}, 1, \underline{\underline{n}}_{0}\right) \in K$ (hence $|\underline{\hat{\eta}}|_{h}=1$ ), to conclude that $q_{0} \notin \mathrm{WF}_{\mathrm{b}, \tilde{\mathcal{D}}}(u)$ it suffices to know that for 
some $0<\delta<\delta_{0}$, with $C_{0} \delta \leq \beta<1$, and for all $q=(x, t, y, z, \hat{\xi}, 1, \hat{\eta}, \hat{\zeta}) \in \Sigma$

$$
\begin{aligned}
\left.\mid \dot{\pi}^{\mathrm{e}}(q)-\exp \left(-\delta W^{\mathrm{b}}\right)\left(q_{0}\right)\right) \mid \leq \beta \delta \text { and }|x(q)| & \leq \beta \delta \\
& \Longrightarrow \pi(q) \notin \mathrm{WF}_{\mathrm{b}, \tilde{\mathcal{D}}}(u) .
\end{aligned}
$$

Remark 9.11 . In the estimate $(9.41), W^{b}$ can be replaced by any $\mathcal{C}^{\infty}$ vector field which reduces to $W^{b}$ at $q_{0}$ since flow to distance $\delta$ along a vector field only depends on the vector field evaluated at the initial point of the flow, up to an error $\mathcal{O}\left(\delta^{2}\right)$. Similarly, changing the initial point of the flow by $\mathcal{O}\left(\delta^{2}\right)$ does not affect the endpoint up to an error $\mathcal{O}\left(\delta^{2}\right)$. Thus, estimate (9.41) can be further rewritten, at the cost of changing $C_{0}$ again, as

$$
\begin{aligned}
\left|\exp \left(\delta W^{\mathrm{b}}\right)\left(\dot{\pi}^{\mathrm{e}}(q)\right)-q_{0}\right| & \leq \beta \delta \text { and }\left|x\left(\exp \left(\delta W^{\mathrm{b}}\right)(q)\right)\right| \leq \beta \delta \\
& \Longrightarrow \pi(q) \notin \mathrm{WF}_{\mathrm{b}, \tilde{\mathcal{D}}}(u) ;
\end{aligned}
$$

here we interchanged the roles of the initial and final points of the flow.

Proof. To prove this result it suffices to modify the proof of the glancing propagation result of [25], with the modifications similar to those leading to the normal propagation above. The key ingredient is Lemma 9.8, which allows the $Q_{i}$ factors, including $Q_{0}$, in the commutator in Corollary 9.6 to be shown to be small, provided the symbol of the commutant is arranged to be supported sufficiently close to the glancing set. Thus, the $L_{0}$ term of Corollary 9.6 dominates the commutator (there is no $\underline{\xi}$ dependence of the commutant in this case near the characteristic set), with principal symbol at $x=0$ given by $2 a H_{h-\tau^{2}} a$.

First take a function $\omega_{0} \in \mathcal{C}^{\infty}\left(S^{*}(\mathbb{R} \times Y)\right)$ which is a sum of squares of $2 l$ $(l=\operatorname{dim} Y)$ homogeneous degree zero functions $\rho_{j}$ :

$$
\omega_{0}=\sum_{j=1}^{2 l} \rho_{j}^{2}, W^{b} \rho_{j}\left(q_{0}\right)=0, \rho_{j}\left(q_{0}\right)=0,
$$

$d \rho_{j}\left(q_{0}\right), j=1, \ldots, 2 l$ linearly independent at $q_{0}$. Since $\operatorname{dim} S^{*}(\mathbb{R} \times Y)=$ $2 l+1, d \rho_{j}\left(q_{0}\right), j=1, \ldots, 2 l$, together with $d t(t$ is also homogeneous of degree zero), span the cotangent space of the $S^{*}(\mathbb{R} \times Y)$, for dimensional reasons (note that $W^{\mathrm{b}} t\left(q_{0}\right) \neq 0$ ). In particular,

$$
\left|\underline{\tau}^{-1} W^{\mathrm{b}} \omega_{0}\right| \leq C_{1}^{\prime} \omega_{0}^{1 / 2}\left(\omega_{0}^{1 / 2}+\left|t-t_{0}\right|\right)
$$

Then extend $\omega_{0}$ to a function on ${ }^{\mathrm{b}} T^{*} M$ (using the trivialization) and set

$$
\omega=\omega_{0}+x^{2} \text {. }
$$

Then the 'naive' estimate, playing an analogous role to (9.15) in the hyperbolic region, is

$$
\begin{aligned}
\left|\underline{\tau}^{-1} H_{p_{0}} \omega\right| & \leq \tilde{C}_{1}^{\prime \prime} \omega^{1 / 2}\left(\omega^{1 / 2}+\left|t-t_{0}\right|+|\hat{\xi}|^{2}+|\hat{\xi}|^{2}\right) \\
& \leq C_{1}^{\prime \prime} \omega^{1 / 2}\left(\omega^{1 / 2}+\left|t-t_{0}\right|+\underline{\tau}^{-2}\left|p_{0}\right|\right)
\end{aligned}
$$


here we used $\left.p_{0}\right|_{x=0}=\underline{\tau}^{2}-|\underline{\xi}|^{2}-|\underline{\eta}|_{h}^{2}-|\underline{\zeta}|_{k}^{2}$, which lets us estimate

$$
\underline{\tau}^{-2}|\underline{\xi}|^{2} \leq C\left(\underline{\tau}^{-2}\left|p_{0}\right|+|x|+\omega_{0}^{1 / 2}+\left|t-t_{0}\right|\right),
$$

for $1-|\hat{\zeta}|_{y}^{2}$ is homogeneous of degree zero and vanishes at $\dot{\mathcal{G}}$ (recall that this last estimate motivates Lemma 9.8). Note that (9.44) is much more precise than (9.15): we have a factor of $\omega^{1 / 2}+\left|t-t_{0}\right|+\tau^{-2}\left|p_{0}\right|$ in addition to $\omega^{1 / 2}$ - this is crucial since we need to get the direction of propagation right.

Finally put

$$
\phi=t-t_{0}+\frac{1}{\beta^{2} \delta} \omega
$$

and define $a=a_{0}$ almost as in (9.17), with $-\hat{\xi}$ replaced by $t-t_{0}$, namely

$$
a=\chi_{0}(2-\phi / \delta) \chi_{1}\left(\left(t-t_{0}+\delta\right) / \beta \delta+1\right) \chi_{2}\left(|\sigma|^{2} / \tau^{2}\right) .
$$

The slight difference is in the argument of $\chi_{1}$, in order to microlocalize more precisely in the 'hypothesis region', i.e. where $u$ is a priori assumed to have no wave front set. This is natural, since for the hyperbolic points we only needed to prove that singularities cannot stay at the boundary, while for glancing points we need to get the correct direction of propagation. We always assume for this argument that $\beta<1$, so on $\operatorname{supp} a$ we have

$$
\phi \leq 2 \delta \text { and } t-t_{0} \geq-\beta \delta-\delta \geq-2 \delta .
$$

Since $\omega \geq 0$, the first of these inequalities implies that $t-t_{0} \leq 2 \delta$, so on $\operatorname{supp} a$

$$
\left|t-t_{0}\right| \leq 2 \delta
$$

Hence,

$$
\omega \leq \beta^{2} \delta\left(2 \delta-\left(t-t_{0}\right)\right) \leq 4 \delta^{2} \beta^{2} .
$$

Moreover, on $\operatorname{supp} d \chi_{1}$,

$$
t-t_{0} \in[-\delta-\beta \delta,-\delta], \omega^{1 / 2} \leq 2 \beta \delta,
$$

so this region lies in the hypothesis region of (9.42) after $\beta$ and $\delta$ are both replaced by appropriate constant multiples.

Now, using (9.44), (9.48), and $\underline{\tau}^{-1} H_{p_{0}}\left(t-t_{0}\right)=2$, we deduce that at $p_{0}=0$,

$$
\begin{aligned}
\underline{\tau}^{-1} H_{p_{0}} \phi & =\underline{\tau}^{-1} H_{p_{0}}\left(t-t_{0}\right)+\frac{1}{\beta^{2} \delta} \underline{\tau}^{-1} H_{p_{0}} \omega \\
& \geq 1-\frac{1}{\beta^{2} \delta} C_{1}^{\prime \prime} \omega^{1 / 2}\left(\omega^{1 / 2}+\left|t-t_{0}\right|\right) \\
& \geq 1-2 C_{1}^{\prime \prime}\left(\delta+\frac{\delta}{\beta}\right) \geq c_{0} / 4>0
\end{aligned}
$$


provided that $\delta<\frac{2}{16 C_{1}^{\prime \prime}}, \frac{\beta}{\delta}>\frac{16 C_{1}^{\prime \prime}}{2}$, i.e. that $\delta$ is small, but $\beta / \delta$ is not too small-roughly, $\beta$ can go to 0 at most as a multiple of $\delta$ (with an appropriate constant) as $\delta \rightarrow 0$. Recall also that $\beta<1$, so there is an upper bound as well for $\beta$, but this is of no significance as we let $\delta \rightarrow 0$. It is also worth remembering that in the hyperbolic region, $\beta$ roughly played the same role as here, but was bounded below by an absolute constant, rather than by a suitable multiple of $\delta$, hence could not go to 0 as $\delta \rightarrow 0$. With this, we can proceed exactly as in the hyperbolic region, so (recalling that $\underline{\tau}>0$ on $\operatorname{supp} a !)$

$$
H_{p_{0}} a^{2}=-b_{0}^{2}+e, b_{0}=\tau^{1 / 2}\left(2 \tau^{-1} H_{p_{0}} \phi\right)^{1 / 2} \delta^{-1 / 2}\left(\chi_{0} \chi_{0}^{\prime}\right)^{1 / 2} \chi_{1} \chi_{2},
$$

with $e$ arising from the derivative of $\chi_{1} \chi_{2}$. Again, $\chi_{0}$ stands for $\chi_{0}\left(2-\frac{\phi}{\delta}\right)$, etc. In view of (9.49) and (9.42) on the one hand, and the fact that $\operatorname{supp} d \chi_{2}$ is disjoint from the characteristic set on the other, both $\operatorname{supp} d \chi_{1}$ and $\operatorname{supp} d \chi_{2}$ are disjoint from $\mathrm{WF}_{\mathrm{b}, \tilde{\mathcal{D}}}(u)$. Thus, $i\left[A^{*} A, \square\right]$ is positive modulo terms that are controlled a priori, so the standard positive commutator argument gives an estimate for $B u$, where $B$ has symbol $b_{0}$. Replacing $a$ by $a \underline{\tau}^{s+1 / 2}$, still gives a positive commutator since $D_{t}$ commutes with $\square$, which now gives (with the new $B) B u \in L^{2}(M, d g)$. In particular $q_{0} \notin \mathrm{WF}_{\mathrm{b}, \tilde{\mathcal{D}}}^{s}(u)$.

Now, applying arguments that go back to [18] we find

Theorem 9.12. If $u \in H_{\tilde{\mathcal{D}}, l o c}^{-\infty}(M)$, then for all $s \in \mathbb{R} \cup\{\infty\}$,

$$
\mathrm{WF}_{\mathrm{b}, \tilde{\mathcal{D}}^{\prime}}^{s}(u) \backslash \mathrm{WF}_{\mathrm{b}, \tilde{\mathcal{D}}^{\prime}}^{s+1}(\square u) \subset \dot{\Sigma}
$$

is a union of maximally extended generalized broken bicharacteristics of $\square$ in $\dot{\Sigma} \backslash \mathrm{WF}_{\mathrm{b}, \tilde{\mathcal{D}}^{\prime}}^{s+1}(\square u)$.

\section{Fiber Dimension 1}

In this section we indicate the changes necessary in the previous sections to accommodate fiber dimension 1. Fortunately, these are quite minor, due to the rather trivial character of 1 dimensional Riemannian geometry. The basic reason for treating fiber dimension 1 separately is that $x^{-1}$ is not bounded from $\tilde{\mathcal{D}}$ to $L^{2}$, and terms with $x^{-1}$ arise throughout the previous section. Here we introduce a class of operators that we call very basic in order to eliminate these terms - this is possible as the metric on the fibers can be put in a rather simple form. ${ }^{4}$

We can assume that all fibers are circles (disconnected fibers can be dealt with similarly). Moreover, one may arrange that the fiber metric is $k(y) d z^{2}$, $d z^{2}$ denoting the standard metric on the circle (corresponding to a circumference of $2 \pi$, say). Correspondingly, locally in the base $Y$ (and all our

\footnotetext{
${ }^{4}$ Another, perhaps more natural way to proceed would be to consider operators that commute with the projection to fiber-constant functions at the boundary.
} 
considerations are local in the base) there is a circle action on $\partial M$, with infinitesimal generator $\partial_{z}$.

Definition 10.1. Let $x^{-1} \mathcal{V}_{\mathrm{e}}(M ; Y)$ be the subspace of $x^{-1} \mathcal{V}_{\mathrm{e}}(M)$ consisting of vector fields $V$ such that $\left[V, \partial_{z}\right] \in \mathcal{V}_{\mathrm{e}}(M)$.

Remark 10.2. $x^{-1} \mathcal{V}_{\mathrm{e}}(M ; Y)$ is not a left $\mathcal{C}^{\infty}(M)$-module, but it is a left $\mathcal{C}_{Y}^{\infty}(M)$-module, where

$$
\mathcal{C}_{Y}^{\infty}(M)=\left\{f \in \mathcal{C}^{\infty}(M):\left.f\right|_{\partial M} \text { is fiber constant }\right\} .
$$

Note also that $x$ is determined by the form of the metric up to multiplication by $a \in \mathcal{C}_{Y}^{\infty}(M)$.

Definition 10.3. We say that $A \in \Psi_{\mathrm{b} \infty}^{m}(M)$ is very basic if it commutes with $\partial_{z}$ at $x=0$, i.e. if $\left[A, \partial_{z}\right] \in x \Psi_{\mathrm{b} \infty}^{m}(M)$.

Remark 10.4. Very basic operators form a $\mathcal{C}_{Y}^{\infty}(M)$-bimodule. Moreover, they form a ring under composition as $\left[A B, \partial_{z}\right]=\left[A, \partial_{z}\right] B+A\left[B, \partial_{z}\right]$. Also, if $A \in \Psi_{\mathrm{b} \infty}^{m}(M)$ is very basic, $x^{-p}\left[A, x^{p}\right] \in \Psi_{\mathrm{b} \infty}^{m-1}(M)$ is also very basic, by the Jacobi identity.

The point of this definition is:

Lemma 10.5. If $A \in \Psi_{\mathrm{b} \infty}^{m}(M)$ is very basic then

(1) (replaces Lemma 8.1) for any $Q \in x^{-1} \mathcal{V}_{\mathrm{e}}(M ; Y)$ there exist very basic $A_{j} \in \Psi_{\mathrm{b}}^{m-1}(M)$ and $Q_{j} \in x^{-1} \mathcal{V}_{\mathrm{e}}(M ; Y), j=1, \ldots, l, A_{0} \in$ $\Psi_{\mathrm{b}}^{m}(M)$ such that

$$
[Q, A]=A_{0}+\sum A_{j} Q_{j}
$$

(2) (replaces Lemma 8.6) there exist $B \in \Psi_{\mathrm{b} \infty}^{m}(M), E \in \Psi_{\mathrm{b} \infty}^{m-1}(M)$ depending continuously on $A$ such that

$$
\left[x^{-1} D_{z}, A\right]=B+E x^{-1} D_{z},
$$

with $E$ very basic, and there exist $B \in \Psi_{\mathrm{b} \infty}^{m}(M), C \in \Psi_{\mathrm{b} \infty}^{m-1}(M)$ depending continuously on $A$ such that

$$
\left[D_{x}, A\right]=B+C D_{x}
$$

and $C$ is very basic.

(3) (replaces Lemma 6.2) if $m=0, A: \tilde{\mathcal{D}} \rightarrow \tilde{\mathcal{D}}$ with norm bounded by a seminorm of $A$ in $\Psi_{\mathrm{b}}^{0}(M)$.

Thus, for very basic operators $x^{-1} D_{z}$ behaves much like $D_{x}$.

Proof. (1,2): As very basic ps.d.o's form a $\mathcal{C}_{Y}^{\infty}(M)$ bimodule, it suffices to check that the conclusion holds for $Q=D_{x}, D_{y_{i}}$ and $Q=x^{-1} D_{z}$ in some local coordinates on $Y$. For $D_{y_{i}}$ this is immediate as $D_{y_{i}}$ is a very basic element of $\Psi_{\mathrm{b}}^{1}(M)$, so $\left[A, D_{y_{i}}\right]$ is a very basic element of $\Psi_{\mathrm{b}}^{m}(M)$.

For $Q=x^{-1} D_{z}$, we compute $\left[x^{-1} D_{z}, A\right]=\left[x^{-1}, A\right] x\left(x^{-1} D_{z}\right)+x^{-1}\left[A, D_{z}\right]$. The second term is in $\Psi_{\mathrm{b} \infty}^{m}(M)$ as $A$ is very basic, while the coefficient of 
$x^{-1} D_{z}, E=\left[x^{-1}, A\right] x \in \Psi_{\mathrm{b} \infty}^{m-1}(M)$ is very basic in view of the previous remark, proving the first line of (2). A similar argument applies to $D_{x}=$ $x^{-1}\left(x D_{x}\right)$, noting that $\left[A, x D_{x}\right] \in x \Psi_{\mathrm{b} \infty}^{m}(M)$ just by virtue of $A \in \Psi_{\mathrm{b} \infty}^{m}(M)$.

(3): As in the proof of Lemma 6.2, this reduces to $[A, V]: \tilde{\mathcal{D}} \rightarrow L^{2}(M)$ being bounded for $A \in \Psi_{\mathrm{b} \infty}^{0}(M)$ and $V \in x^{-1} \mathcal{V}_{\mathrm{e}}(M ; Y)$. By (1), this follows from $A_{0}, A_{j}$ being bounded on $L^{2}$ while $Q_{j}: \tilde{\mathcal{D}} \rightarrow L^{2}(M)$.

Of course, we need to know that there is a plentiful supply of very basic operators.

Lemma 10.6. Suppose that $a \in S^{m}\left({ }^{b} T^{*} M\right)$ is such that $\left.a\right|_{x=0}$ is invariant under the circle action. Then there exists $A \in \Psi_{\mathrm{b}}^{m}(M)$ very basic such that $\sigma(A)=a$.

Proof. Using a product decomposition of $M$ near $\partial M$, extend the circle action to a neighborhood $O$ of $\partial M$. Let $A^{\prime} \in \Psi_{\mathrm{b}}^{m}(M)$ be a quantization of $a$, so $\sigma\left(A^{\prime}\right)=a$, and let $A$ be the average of $A^{\prime}$ under the circle action, i.e. $A^{\prime \prime}=(2 \pi)^{-1} \int_{S^{1}} A_{\theta} d \theta$, where $A_{\theta}$ is the conjugate of $A$ by pull-back by translation by $\theta$. Due to the averaging, $A$ commutes with the infinitesimal generator of the circle action, $\partial_{z}$, so $A^{\prime \prime}$ is indeed very basic. Moreover, $\left.\sigma\left(A^{\prime \prime}\right)\right|_{x=0}=\left.a\right|_{x=0}$ as $a$ is invariant under the circle action, so the symbol of $A_{\theta}$ at $x=0$ is also $a$. Now let $\tilde{A} \in x \Psi_{\mathrm{b}}^{m}(M)$ be such that $\sigma(\tilde{A})=a-\sigma\left(A^{\prime \prime}\right)$; then $A=A^{\prime \prime}+\tilde{A}$ has all the desired properties.

With the help of this lemma, all proofs in Section 8 go through, provided we use commutants that are very basic.

Corollary 10.7. (Cf. Lemma 9.2.) With a trivialization in which the fiber metric at $x=0$ is $k(y) d z^{2}$, suppose that $\tilde{a}$, a are as in Lemma 9.2, where $|\zeta|^{2}$ stands for the lift of the standard metric on the circle $Z$. Let $A$ be as in the previous lemma. Then $\left[\partial_{z}, A\right]=B \in x \Psi_{\mathrm{b}}^{m}(M)$ and $\mathrm{WF}_{\mathrm{b}}^{\prime}(B) \cap \dot{\Sigma}=\emptyset$.

Proof. We merely need to observe that $\left.a\right|_{x=0}$ is invariant under the circle action, and apply the previous lemma to deduce $\left[\partial_{z}, A\right]=B \in x \Psi_{\mathrm{b}}^{m}(M)$. The wave front set statement follows from the construction of $A$ : it holds (in a uniform fashion) for each $A_{\theta}$.

With the notation of the paragraph preceding Lemma 9.3, since $d g=J \nu$ with $J \in \mathcal{C}_{Y}^{\infty}(M)$, as $\left.\partial_{z} \log J\right|_{x=0}=0$, Lemma 9.3 can be strengthened as follows:

Lemma 10.8. With $A=A^{\dagger}$ as in $\S 9$

$$
\begin{aligned}
& {\left[W, A^{*} A\right]=B+F, B \in x \Psi_{\mathrm{b}}^{2 m}(M), F \in x \Psi_{\mathrm{b}}^{2 m-1}(M)} \\
& \mathrm{WF}_{\mathrm{b}}^{\prime}(B) \cap \dot{\Sigma}=\emptyset, \sigma(F)=a\{a, W \log J\},
\end{aligned}
$$

with both $B$ and $F$ depending continuously on $A$. Here $W \log J \in x \mathcal{C}^{\infty}(M)$ is lifted to ${ }^{\mathrm{b}} T^{*} M$ by the bundle projection. 
Now Lemma 9.5 can be strengthened to include a statement that $\kappa_{i j}$ is very basic, i.e. $\kappa_{i j} \in \mathcal{C}_{Y}^{\infty}(M)$.

Then Corollary 9.6 remains valid even if the sums are so that $Q_{0}=x^{-1}$ is excluded from them, and $L_{i j}$ are very basic.

Consequently, the proofs in the rest of Section 9 go through.

\section{Edge PROPAGATION}

The flow along (2.4) in the boundary, i.e. at $x=0$, is explicitly solvable in ${ }^{\mathrm{e}} T_{\partial M}^{*} M$ : we have

$$
\xi=|\zeta| \tan (|\zeta| s+C)
$$

while $|\zeta|$ is conserved, $(z, \zeta /|\zeta|)$ undergo geodesic flow at speed $|\zeta|$ with respect to the base metric on the boundary, and

$$
\tau=A \sec (|\zeta| s+C), \quad \eta=B \sec (|\zeta| s+C) .
$$

Thus along maximally extended integral curves, $(z, \zeta /|\zeta|)$ undergoes time- $\pi$ geodesic flow and $(\tau, \eta)$ traverse a line through the origin in $\mathbb{R}^{b+1}$. Since the Hamilton vector field along these curves is nowhere vanishing, we conclude from the arguments of [16] that for any $k, l, \mathrm{WF}_{\mathrm{e}}{ }^{k, l} u$ is a union of such integral curves. The Hamilton vector field vanishes at the endpoints of these integral curves, at the codimension-two corner of the radial compactification of ${ }^{\mathrm{e}} T^{*} X$, hence the question remaining is how the interior wavefront set estimates on $u$ extend to the corner, and thence into and out of ${ }^{\mathrm{e}} T_{\partial X}^{*} X$. In fact, we can do a (crucial) bit more, obtaining propagation of coisotropic regularity, i.e. regularity under $\mathcal{A}^{k}$, along the flow discussed above.

Theorem 11.1. Let $u \in H_{\mathrm{e}}^{-\infty, l}(I \times X)$ be a distributional solution to the wave equation with $\square u=0, \bar{t} \in I \subset \mathbb{R}$ with $I$ open.

(1) Let $m>l+f / 2$. Given $p \in \dot{\mathcal{H}}_{I}$, if $\left(\mathcal{F}_{I, p} \backslash \partial M\right) \cap \mathrm{WF}^{m} A u=\emptyset$, for all $A \in \mathcal{A}^{k}$ then $p \notin \mathrm{WF}_{\mathrm{e}}{ }^{m, l^{\prime}} B u$ for all $l^{\prime}<l$ and all $B \in \mathcal{A}^{k}$.

(2) Let $m<l+f / 2$. Given $p \in \dot{\mathcal{H}}_{O}$, if a neighborhood $U$ of $p$ in $\left.{ }^{\mathrm{e}} S^{*}\right|_{\partial M} M$ is such that $\mathrm{WF}_{\mathrm{e}}{ }^{m, l}(A u) \cap U \subset \partial \mathcal{F}_{O}$ for all $A \in \mathcal{A}^{k}$ then $p \notin \mathrm{WF}_{\mathrm{e}}{ }^{m, l}(B u)$ for all $B \in \mathcal{A}^{k}$.

Remark 11.2. For any point $p \in{ }^{\mathrm{e}} T_{\partial M}^{*} M \backslash\{\zeta=0\}$ there is an element of $\mathcal{A}^{k}$ elliptic there, hence (1), with $k=\infty$, shows that solutions with (infinite order) coisotropic regularity have no wavefront set in ${ }^{\mathrm{e}} T_{\partial M}^{*} M \backslash\{\zeta=0\}$. Indeed this result holds microlocally in the edge cotangent bundle. Note that the set $\{x=0, \zeta=0\}$ is just the set of radial points for the Hamilton vector field.

As the proof follows quite closely that of Theorem 8.1 of [19], we shall be somewhat concise here. However, as the flowout cannot be put in a simple model form as in [19], we need a lemma adapted from [8] to handle factors from $\mathcal{A}^{m}$, replacing the powers of the fiber Laplacian used in [19]. This is stated in the Appendix, in Proposition A.6. 
Proof of Theorem 11.1. We only consider part (1) of the Theorem; the proof of (2) is completely analogous, cf. [19].

Let $p=(\bar{t}, \bar{y}, \bar{z}, \bar{\tau}, \bar{\xi}, \bar{\eta}) \in \dot{\mathcal{H}}_{I}$. We begin by constructing a localizer in the fast variables. Let

$$
\Upsilon:{ }^{\mathrm{e}} T_{\partial M}^{*}(M) \backslash\{\xi=0\} \rightarrow Z
$$

be locally defined by

$$
\Upsilon(q)=z\left(\exp _{z_{0}, \zeta_{0}} s_{\infty} H_{q}\right), \quad s_{\infty}=\frac{\operatorname{sgn} \xi}{|\zeta|_{K_{q}}} \arctan \frac{|\zeta|}{\xi} .
$$

where $q \in{ }^{\mathrm{e}} T_{\partial M}^{*}(M)$ has coordinates $\left(t_{0}, y_{0}, z_{0}, \tau_{0}, \xi_{0}, \eta_{0}, \zeta_{0}\right)$. This map simply takes a point over the boundary to its limit point in the fiber variables along the forward bicharacteristic flow, hence on the boundary, we certainly have $\Upsilon_{*}(H)=0$. We now extend $\Upsilon$ smoothly to the interior of ${ }^{\mathrm{e}} T^{*} M$, thus obtaining a map satisfying

$$
\Upsilon_{*}(H)=O(x) .
$$

Let $a_{i}$ be homogeneous degree zero defining functions for $\overline{\mathcal{F}}_{I}$ as in Section 4. (In fact, for the proof of (1), one could use $\zeta_{i}$ in place of $a_{i}$, as one is only concerned about positivity at $x=0$, where one can take $a_{i}=\zeta_{i}$, but for the proof of (2), the use of $a_{i}$ is important.)

Now fix any sufficiently small neighborhood $U$ of $p$ in ${ }^{\mathrm{e}} T^{*} M$. There exists $\delta>0$ such that

$$
\left\{x<\delta,\left|a_{i}\right|<\delta,|\eta / \tau-\bar{\eta} / \bar{\tau}|^{2}+|y-\bar{y}|^{2}+d(\Upsilon, \bar{z})^{2}+|t-\bar{t}|^{2}<\delta\right\} \subset U .
$$

There exists $\beta$ such that

$$
H\left(|\eta / \tau-\bar{\eta} / \bar{\tau}|^{2}+|y-\bar{y}|^{2}+d(\Upsilon, \bar{z})^{2}+|t-\bar{t}|^{2}-\beta x\right)>0
$$

on $U$, since the derivative falling on each term in the cut-off is $O(x)$, and $H(x)=\xi x+O\left(x^{2}\right)$, i.e. is bounded above on $U$ by a negative multiple of $x$.

We now need some cut-off functions. Let $\psi$ be nonnegative, nonincreasing, and supported in $(-\infty, \epsilon)$, and $\chi \in \mathcal{C}^{\infty}(\mathbb{R})$ be nonnegative, nondecreasing, and supported in $(\epsilon, \infty)$. We may further arrange that $\psi, \chi,-\psi^{\prime}$ and $\chi^{\prime}$ are squares of smooth functions. We also let $\phi \in \mathcal{C}_{c}^{\infty}([0, \epsilon))$ be nonnegative, nonincreasing, identically 1 near 0 , with $\phi,-\phi^{\prime}$ the squares of a smooth function.

Set

$$
\begin{aligned}
& a_{m, l}^{2}=\chi( \pm \tau) \chi( \pm \xi) \phi(x) \phi\left(\epsilon^{-1} \sum a_{j}^{2}\right) \psi\left(p^{2}(\cdot) / \tau^{4}\right) \\
& \quad \psi\left(\left(|\eta / \tau-\bar{\eta} / \bar{\tau}|^{2}+|y-\bar{y}|^{2}+d(\Upsilon, \bar{z})^{2}+|t-\bar{t}|^{2}-\beta x\right)( \pm \tau)^{m} x^{l},\right.
\end{aligned}
$$

with $\pm=\operatorname{sgn} \bar{\xi} \bar{\tau}$

Note that applying $H$ to $\tau^{m} x^{l}$ on the support of $a_{m, l}$ gives a main term $(m+l) \xi^{m} x^{l}$, hence this term is positive provided $m+l>0$. As long as $\epsilon$ is sufficiently small that $(\beta+1) \epsilon<\delta$, the support of the symbol lies in $U$, hence choosing $\epsilon$ sufficiently small ensures the positivity of the term in 
$H\left(a_{m, l}^{2}\right)$ coming from the last cut-off term. The cut-off factors involving $\chi$ are harmless as the support of the Hamilton vector field applied to these intersects the characteristic set inside ${ }^{\mathrm{e}} T^{*} M$ in a compact set. Finally, the cut-off terms involving $\phi$ have the following properties: the Hamilton derivative of $\phi(x)$ supported in the interior of ${ }^{\mathrm{e}} T^{*} M$, while the Hamilton derivative of $\phi\left(\epsilon^{-1} \sum a_{j}^{2}\right)$ is supported away from $\overline{\mathcal{F}}_{I}$.

Using the above observations, provided $m+l>0$, by choosing $\epsilon$ sufficiently small, we can ensure that

$$
H\left(a_{m, l}^{2}\right)= \pm\left(a^{\prime}\right)^{2} \pm \sum_{j} b_{j}^{2}+e+c+k
$$

where supp $e \subset T^{*} X^{\circ}$, supp $c$ is compact in ${ }^{\mathrm{e}} T^{*}(M)$ and $\Sigma \cap \operatorname{supp}(k)=\emptyset$.

For $k=0$, the proof is finished with a positive commutator argument as in [19, Theorem 8.1]. For arbitrary $k$, we can now use Proposition A.6 to finish the proof inductively.

Remark 11.3. The reason for the appearance of $f / 2$ in the statement of the theorem is that in order to prove the theorem, we need to use $a_{r, s}$ with $r=m-\frac{1}{2}, s=-l-\frac{f-1}{2}$. Indeed, the commutator of $A_{r, s}^{*} A_{r, s}$ (with $A_{r, s} \in \Psi_{\mathrm{e}}^{r, s}(M)$ having principal symbol $\left.a_{r, s}\right)$ with $\square$ is in $\Psi_{\mathrm{e}}^{2 r-1,2 s-2}(M)$. As in the commutator argument we use the metric density $d g$ (to make $\square$ formally self-adjoint), which is $x^{f+1}$ times a non-degenerate smooth bdensity (with respect to which $H_{\mathrm{e}}^{m, l}(M)$ is weighted), a principal term in this commutator of the form $\left(A^{\prime}\right)^{*} A^{\prime}$ provides a bound for the $H_{\mathrm{e}}^{m, l}(M)$ norm of $u$ provided that $2 m-(2 r-1)=0,2 l+(2 s-2)+(f+1)=0$, leading to the stated values of $r$ and $s$ in terms of $m$ and $l$. As in order to obtain a positive commutator we need $r+s>0$ (or $<0$ in the outgoing region), we deduce that $m>l+\frac{f}{2}$ (resp. $m<l+\frac{f}{2}$ ) must be satisfied.

Moreover, the reason for $l^{\prime}<l$ in the statement of the theorem is the possible need for an interpolation argument, in case the a priori regularity of $u$ is weak, i.e. $u \in H_{\mathrm{e}}^{q, l}$ with $q$ too small (possibly negative). This is because, due to the standard error terms, the regularity of $u$ can only be improved by $1 / 2$ order at a time, so already $m=q+1 / 2$ would have to satisfy $m>l+\frac{f}{2}$, i.e. we would need $q>l+\frac{f-1}{2}$. In particular, if $q>l+\frac{f-1}{2}$ is satisfied, i.e. if we have this much a priori regularity, we can take $l^{\prime}=l$.

\section{Propagation of Coisotropic Regularity}

Theorem 12.1. Let $p \in \dot{\mathcal{H}}, \epsilon>0$ and $k \in \mathbb{N}$. Then there is $k^{\prime}$ (depending on $k$ and $\epsilon$ ) such that if $\square u=0$ and $u$ has coisotropic regularity of order $k^{\prime}$ relative to $H^{s}$ (on the coisotropic $\dot{\mathcal{F}}_{I}$ ) near $\dot{\mathcal{F}}_{I, p}$ strictly away from $\partial M$, then $u$ has coisotropic regularity of order $k$ relative to $H^{s-\epsilon}$ (on the coisotropic $\dot{\mathcal{F}}_{O}$ ) near $\dot{\mathcal{F}}_{O, p}$, strictly away from $\partial M$.

In particular, if $\square u=0$ and $u$ is coisotropic (i.e. has infinite regularity) relative to $H^{s}$ (on the coisotropic $\dot{\mathcal{F}}_{I}$ ) near $\dot{\mathcal{F}}_{I, p}$ strictly away from $\partial M$, then 
for all $\epsilon>0, u$ is coisotropic relative to $H^{s-\epsilon}$ (on the coisotropic $\dot{\mathcal{F}}_{O}$ ) near $\dot{\mathcal{F}}_{O, p}$, strictly away from $\partial M$.

Explicitly, with the argument presented below, one can take $k^{\prime}=k$ if $\epsilon>1 / 2$, and any $k^{\prime}>\frac{1}{2 \epsilon} k$ if $\epsilon \leq 1 / 2$.

Proof. First we show that the result holds with $H^{s-0}$ replaced by $H^{s-1 / 2-0}$. Indeed, by Theorem $9.7, u$ is $\mathcal{C}^{\infty}$ microlocally near $\dot{\mathcal{F}}_{O, p}$ strictly away from $\partial M$ provided it is $\mathcal{C}^{\infty}$ microlocally near $\dot{\mathcal{F}}_{I, p}$ away from $\partial M$, i.e.

$$
\mathrm{WF}(u) \cap\left(\dot{\mathcal{F}}_{I, p} \backslash \partial \dot{\mathcal{F}}_{I, p}\right)=\emptyset \Longrightarrow \mathrm{WF}(u) \cap\left(\dot{\mathcal{F}}_{O, p} \backslash \partial \dot{\mathcal{F}}_{O, p}\right)=\emptyset .
$$

In particular, $u$ is coisotropic as stated, provided that $\operatorname{WF}(u) \cap\left(\dot{\mathcal{F}}_{I, p} \backslash\right.$ $\left.\partial \dot{\mathcal{F}}_{I, p}\right)=\emptyset$. Thus, we we may assume that $u$ is microlocalized near $\dot{\mathcal{F}}_{I, p}$ and away from $\partial M$ for $t<t(p)$, hence that $u$ and $\mathcal{A}^{k} u$ lie in $H_{\mathrm{loc}}^{s}\left(M^{\circ}\right)$ for $t<t(p)$ (with loc indicating locally in time). It is convenient to normalize $s$ to make Proposition 5.4 easier to use. Let $\Theta_{s}$ have Schwartz kernel

$$
\kappa\left(\Theta_{s}\right)\left(t, t^{\prime}\right)=\psi\left(t-t^{\prime}\right) \kappa\left(\left|D_{t}\right|^{s}\right)\left(t, t^{\prime}\right)
$$

where $\psi(t)$ is a smooth function of compact support, equal to one near $t=0$ (cf. [19]). Since applying $\Theta_{-s}$ to $u$ preserves the hypotheses, except replacing $s$ by 0 , and then the conclusion is preserved (shifting $s$ back) upon the application of $\Theta_{s}$, we may apply Proposition 5.4, to conclude that $u \in H_{\mathrm{e}, \mathrm{loc}}^{s, s-\frac{f+1}{2}}(M)$ for $t<t(p)$, hence for all $t$.

Then Theorem 11.1 part (1) can be applied, first with $l=s-\frac{f+1}{2}$, $m=s-\frac{1}{2}+0$ near incoming points (so $m>l+\frac{f}{2}$ ), to conclude that $u$ is coisotropic of order $k$ relative to $H_{\mathrm{e}}^{s-\frac{1}{2}+0, s-\frac{f+1}{2}-0}(M)$ near $\partial \dot{\mathcal{F}}_{I}$. Note that if $U$ is a sufficiently small neighborhood of $\dot{\mathcal{H}}_{I}=\partial \dot{\mathcal{F}}_{I}$ in ${ }^{\mathrm{e}} T_{\partial M}^{*} M$, this implies that in fact $u$ is microlocally in $H_{\mathrm{e}}^{s+k-\frac{1}{2}+0, s-\frac{f+1}{2}-0}(M)$ on $U \backslash \dot{\mathcal{H}}_{I}$, since $\mathcal{M}$ has elliptic elements near each point in $U \backslash \dot{\mathcal{H}}_{I}$. Since bicharacteristics $\gamma$ in $\dot{\pi}^{-1}(\dot{\mathcal{H}}) \backslash\left(\dot{\mathcal{H}}_{I} \cup \dot{\mathcal{H}}_{O}\right) \subset{ }^{\mathrm{e}} T_{\partial M}^{*} M$ tend to $\dot{\mathcal{H}}_{I}$, resp. $\dot{\mathcal{H}}_{O}$, as the parameter along $\gamma$ tends to $\pm \infty$, the standard (non-radial) propagation of singularities, [19, Theorem 8.1(ii)], yields that $u$ is coisotropic of order $k$ relative to $H_{\mathrm{e}}^{s-\frac{1}{2}+0, s-\frac{f+1}{2}-0}(M)$ at $\dot{\pi}^{-1}(\dot{\mathcal{H}}) \backslash \dot{\mathcal{H}}_{O}$-indeed, $u$ is simply in $H_{\mathrm{e}}^{s+k-\frac{1}{2}+0, s-\frac{f+1}{2}-0}(M)$ microlocally on $\dot{\pi}^{-1}(\dot{\mathcal{H}}) \backslash\left(\dot{\mathcal{H}}_{I} \cup \dot{\mathcal{H}}_{O}\right)$. Applying Theorem 11.1 with $m=s-\frac{1}{2}-0, l=s-\frac{f+1}{2}-0$ (the two small constants in the -0 are chosen so that $m<l+\frac{f}{2}$ ), we conclude that $u$ is coisotropic of order $k$ relative to $H_{\mathrm{e}}^{s-\frac{1}{2}-0, s-\frac{f+1}{2}-0}(M)$ near $\dot{\mathcal{F}}_{O}$, as claimed.

On the other hand, $u$ is in $H^{s}$ along $\dot{\mathcal{F}}_{O, p}$ by Theorem 9.7. Hence the theorem follows by the interpolation result of the following lemma.

Lemma 12.2. Suppose that $u$ is in $H^{s}$ microlocally near some point $q$ away from $\partial M$, and it is coisotropic of order $N$ relative to $H^{m}$ near $q$ with $s>m$. 
Then for $\epsilon>0$ and $k<\frac{\epsilon N}{s-m}, u$ is coisotropic of order $k$ relative to $H^{s-\epsilon}$ near $q$.

In particular, if $u$ is in $H^{s}$ microlocally near some point $q$ away from $\partial M$ and $u$ is coisotropic (of order $\infty$, that is) relative to $H^{m}$ near $q$ with $s>m$, then $u$ is coisotropic relative to $H^{s-\epsilon}$ for all $\epsilon>0$.

Proof. If $Q \in \Psi^{0}(M)$ and $\mathrm{WF}^{\prime}(Q)$ lies sufficiently close to $q$, then the hypotheses are globally satisfied by $u^{\prime}=Q u$. Moreover, being coisotropic, locally $\mathcal{F}$ can be put in a model form $\zeta=0$ by a symplectomorphism $\Phi$ in some canonical coordinates $(y, z, \eta, \zeta)$, see [9, Theorem 21.2.4] (for coisotropic submanifolds one has $k=n-l, \operatorname{dim} S=2 n$, in the theorem). Further reducing $\operatorname{WF}^{\prime}(Q)$ if needed, and using an elliptic 0th order Fourier integral operator $F$ with canonical relation given by $\Phi$ to consider the induced problem for $v=F u^{\prime}=F Q u$, we may thus assume that $v \in H^{s}$, and $D_{z}^{\alpha} v \in H^{m}$ for all $\alpha$, i.e. $\left\langle D_{z}\right\rangle^{N} v \in H^{m}$. Considering the Fourier transform $\hat{v}$ of $v$, we then have $\langle\eta, \zeta\rangle^{s} \hat{v} \in L^{2},\langle\eta, \zeta\rangle^{m}\langle\zeta\rangle^{N} \hat{v} \in L^{2}$. But this implies $\langle\eta, \zeta\rangle^{m \theta+s(1-\theta)}\langle\zeta\rangle^{N \theta} \hat{v} \in L^{2}$ for all $\theta \in[0,1]$ by interpolation (indeed, in this case by Hölder's inequality). In particular, taking $\theta=\frac{\epsilon}{s-m}$, $\langle\eta, \zeta\rangle^{s-\epsilon}\langle\zeta\rangle^{k} \hat{v} \in L^{2}$ if $k<\frac{N \epsilon}{s-m}$, and the lemma follows.

\section{Geometric theorem}

We now prove Theorem 1.3, using as our main ingredients Theorem 12.1 and a duality argument.

Let $U_{1}$ be a small open neighborhood of a single point $w \in \mathcal{F}_{O, p}^{\circ}$ such that all points in $\mathcal{F}_{O} \cap U_{1}$ have a distance from $\partial M$ between $0.9 d_{0}$ and $d_{0}$ for some small $d_{0}$. Pick any time $T$ greater than $d_{0}$, so that all interactions of $U_{1}$ with the boundary occur under backward generalized bicharacteristic flow for time less than $T$.

Let $U_{0}^{G}$ and $U_{0}^{D}$ now denote two open sets in $T^{*} M^{\circ}$ such that $U_{0}^{D}$ contains the whole time- $T$ backward flowout of $U_{1}$ in the sense of generalized broken bicharacteristics. $U_{0}^{G}$, by contrast, must contain only the geometric backward flow from $U_{1}$ (see $\S 7$ for the definitions of these flows). By hypothesis, we may choose $U_{0}^{D}$ such that the nonfocusing condition holds relative to $H^{s}$ on $U_{0}^{D}$. We may split the initial data into a piece microsupported in $U_{0}^{G}$ and supported away from a boundary, and a remainder. The former piece, by hypothesis, is globally in $\mathcal{C}\left(\mathbb{R} ; \mathcal{D}_{s}\right)$, hence satisfies the conclusion of the theorem. Thus it suffices to consider only the latter piece, which has no wavefront set of any order in $U_{0}^{G}$.

Let $r<s$. Let $A_{i}, i=1, \ldots, N$, denote first-order pseudodifferential operators, generating $\mathcal{M}$ as above, but now locally over a large set in $M^{\circ}$, and with kernels compactly supported in $M^{\circ}$. Let $B_{0}$ and $B_{1}$ be pseudodifferential operators of order 0 , compactly supported in $M^{\circ}$, and with microsupport in $U_{0}^{D}$ resp. $U_{1}$, such that $B_{1}$ is elliptic at $w$ and such that $B_{0}$ is elliptic on the time- $T$ backward flowout of $\mathrm{WF}^{\prime} B_{1}$ along generalized broken bicharacteristics. Let $U$ denote the forward time-evolution operator, taking 
wave equation solutions on $[0, \delta]$ to wave equation solutions on $[T-\delta, T]$. Theorem 12.1, together with time reversal symmetry, gives

$$
\begin{aligned}
& \sum_{|\alpha| \leq k^{\prime}}\left\|\Theta_{-r+1} A^{\alpha} B_{1} U(u)\right\|_{\tilde{\mathcal{D}}([T-\delta, T])}^{2}<\infty \\
\Longrightarrow & \sum_{|\alpha| \leq k}\left\|\Theta_{-r+1-\epsilon} A^{\alpha} B_{0} u\right\|_{\tilde{\mathcal{D}}([0, \delta])}^{2}<\infty,
\end{aligned}
$$

with $\Theta$. defined by (12.1). Thus, if we define Hilbert spaces

$$
\mathrm{H}_{0}=\left\{u \in \tilde{\mathcal{D}}([0, \delta]): \square u=0, \sum_{|\alpha| \leq k}\left\|\Theta_{-r+1-\epsilon} A^{\alpha} B_{0} u\right\|_{\tilde{\mathcal{D}}([0, \delta])}^{2}<\infty\right\}
$$

and

$$
\mathrm{H}_{1}=\left\{u \in \tilde{\mathcal{D}}([T-\delta, T]): \square u=0, \sum_{|\alpha| \leq k^{\prime}}\left\|\Theta_{-r+1} A^{\alpha} B_{1} u\right\|_{\tilde{\mathcal{D}}([T-\delta, T])}^{2}<\infty\right\},
$$

we have $U^{-1}: \mathrm{H}_{1} \rightarrow \mathrm{H}_{0}$. Hence by unitarity of $U$ with respect to the norm on $\tilde{\mathcal{D}}, U: \mathrm{H}_{0}^{*} \rightarrow \mathrm{H}_{1}^{*}$, where the dual spaces are taken with respect to energy norm, i.e. the norm on $\tilde{\mathcal{D}}$. Now

$$
\mathrm{H}_{0}^{*}=\left\{u \in \tilde{\mathcal{D}}([0, \delta]): \square u=0, u=\sum_{|\alpha| \leq k}\left(A^{\alpha}\right)_{\mathcal{D}}^{*}\left(B_{0}\right)_{\mathcal{D}}^{*} \Theta_{-r+1-0} v_{\alpha}, v_{\alpha} \in \tilde{\mathcal{D}}\right\}
$$

and

$$
\mathrm{H}_{1}^{*}=\left\{u \in \tilde{\mathcal{D}}([T-\delta, T]): \square u=0, u=\sum_{|\alpha| \leq k^{\prime}}\left(A^{\alpha}\right)_{\mathcal{D}}^{*}\left(B_{1}\right)_{\mathcal{D}}^{*} \Theta_{-r+1} v_{\alpha}, v_{\alpha} \in \tilde{\mathcal{D}}\right\}
$$

Thus since $u$ satisfies the nonfocusing condition of degree $k$ w.r.t. $H^{s}$ for $t \in[0, \delta]$ on $U_{0}^{D}$, it lies in $\mathrm{H}_{0}^{*}$; hence $U(u) \in \mathbf{H}_{1}^{*}$, i.e. $u$ also satisfies the nonfocusing condition of degree $k^{\prime}$ w.r.t. $H^{r}$ for $t \in[T-\delta, T]$ on $U_{1}$. (Here we have used Lemma 5.6 to see that lying in the range of $\left(A^{\alpha}\right)_{\mathcal{D}}^{*}$ yields nonfocusing; recall that the $\mathcal{D}$ subscript means adjoint with respect to the $\mathcal{D}$ inner product.) So microlocally near $w$ and for $t \in[T-\delta, T]$,

$$
u \in \sum_{|\alpha| \leq k^{\prime}} A^{\alpha}\left(H^{r}\right) .
$$

On the other hand, since WF $u \cap U_{0}^{G}=\emptyset$, Theorem 11.1 shows that there exists $\beta \in \mathbb{R}$ such that microlocally near $w$ and for $t \in[T-\delta, T]$,

$$
A^{\alpha} u \in H^{\beta} \quad \forall \alpha
$$

(The particular choice of $\beta$ is dependent on the background regularity of the solution.) We can interpolate (13.1) and (13.2) similarly to Lemma 12.2; the only difference is that one has $\langle\eta, \zeta\rangle^{r}\langle\zeta\rangle^{-k^{\prime}} \hat{v} \in L^{2}$ rather than $\langle\eta, \zeta\rangle^{s} \hat{v} \in L^{2}$, with the notation of that lemma. This shows that near microlocally $w$, for $t \in[T-\delta, T], u \in H^{r-0}$. Since $r<s$ is arbitrary, this proves Theorem 1.3. 


\section{Applications to Lagrangian data}

An important class of examples of solutions satisfying the nonfocusing condition is given by choosing as Cauchy data Lagrangian distributions in $X^{\circ}$, with respect to Lagrangian manifolds transverse to the coisotropic submanifold $\mathcal{F}$ obtained by flowout from the boundary. For the proof of the proposition below it is useful to have the following lemma which puts the coisotropic and Lagrangian manifolds into model form, and which seems to be well-known although it is hard to find a published reference.

Lemma 14.1. Suppose that $\mathcal{F}$ is coisotropic of codimension $k, \mathcal{L}$ is Lagrangian, and $\mathcal{F}$ and $\mathcal{L}$ are transverse. Let $\omega$ denote the standard symplectic form on $T^{*} \mathbb{R}^{n}$, and write $\mathbb{R}^{n}=\mathbb{R}_{y}^{n-k} \times \mathbb{R}_{z}^{k}$, with dual coordinates $(\eta, \zeta)$. Then there is a local symplectomorphism mapping $\mathcal{F}$, resp. $\mathcal{L}$ to $\{\zeta=0\}$, resp. $\{y=0, z=0\}$ in $\left(T^{*} \mathbb{R}^{n}, \omega\right)$.

If in addition $\mathcal{F}$ and $\mathcal{L}$ are conic and the canonical one-form does not vanish on $T_{q} \mathcal{F}$, with $q \in \mathcal{F} \cap \mathcal{L}$ then there is a homogeneous symplectomorphism mapping a neighborhood of $q$ into $\left(T^{*} \mathbb{R}^{n}, \omega\right)$, equipped with the standard (fiberwise) $\mathbb{R}^{+}$-action, such that $\mathcal{F}$, resp. $\mathcal{L}$ are mapped into $\{\zeta=0\}$, resp. $\{y=0, z=0\}$.

Proof. By [9, Theorem 21.2.4] (and the subsequent remark), we may assume that $\mathcal{F}$ is given by $\{\zeta=0\}$ in $\left(T^{*} \mathbb{R}^{n}, \omega\right)$. As $\mathcal{L}$ is transverse to $\mathcal{F}, d \zeta_{j}$, $j=1, \ldots, k$, restrict to be linearly independent on $\mathcal{L}$ at points in $\mathcal{L} \cap$ $\mathcal{F}$. Moreover, for $q \in \mathcal{L} \cap \mathcal{F}$, we may assume that $d \eta_{j}(q), j=1, \ldots, n-$ $k$, together with $d \zeta_{j}(q)$, are linearly independent on $\mathcal{L}$. Indeed, a linear combination $\sum c_{j} \partial_{z_{j}}$ cannot be tangent to $\mathcal{L}$ at $q$, as it is the image of $\sum c_{j} d \zeta_{j}$ under the Hamilton map; for a Lagrangian submanifold the tangent space is the image of the conormal bundle under the Hamilton map, and we just established that $\sum c_{j} d \zeta_{j}(q)$ is not an element of the conormal bundle of $\mathcal{L}$. By a symplectic linear transformation in $T^{*} \mathbb{R}^{n-k}$ that switches the roles of some components of $d y$ and $d \eta$ we can arrive at the stated situation.

Thus, $\mathcal{L}$ is locally a graph over $\{0\} \times\left(\mathbb{R}^{n}\right)_{\eta, \zeta}^{*}$, i.e. on $\mathcal{L}, y=Y(\eta, \zeta)$, $z=Z(\eta, \zeta)$. As $\mathcal{L}$ is Lagrangian with respect to $\omega=\sum d \eta_{j} \wedge d y_{j}+\sum d \zeta_{j} \wedge d z_{j}$, it follows that $\mathcal{L}$ is locally the graph of the differential of a function $F$ : $\left(\mathbb{R}^{n}\right)_{\eta, \zeta}^{*} \rightarrow \mathbb{R}$, i.e. $Y=d_{\eta} F, Z=d_{\zeta} F$. The map

$$
(y, z, \eta, \zeta) \mapsto\left(y-d_{\eta} F, z-d_{\zeta} F, \eta, \zeta\right)
$$

is a local symplectomorphism, it preserves $\mathcal{F}=\{\zeta=0\}$, and maps $\mathcal{L}$ to $\{y=0, z=0\}$ as desired.

For the conic version, one may apply [9, Theorem 21.2.4], so one can assume that $\mathcal{F}$ is given by $\zeta=0$. We may assume that $y(q)=0 ; \eta(q) \neq 0$ since $q$ does not lie in the zero section. Now, the span of the $\partial_{z_{j}}$ intersects $T_{q} \mathcal{L}$ trivially as above. Let $V^{*}$ be the span of the $\sum c_{j} d \eta_{j}(q)$ which lie in $N_{q}^{*} \mathcal{L}$, i.e. for which the image $V$ under the Hamilton map $\sum c_{j} \partial_{y_{j}} \in T_{q} \mathcal{L}$. Taking $W$ to be a complementary subspace to $V$ in the span of the $d y_{j}$, we 
see that functions with differentials in $V$ plus functions with differentials in $W^{*}$, together with the $\zeta_{j}$, give coordinates on $\mathcal{L}$ locally. Moreover, by the conic hypothesis, $W$ is at least one-dimensional. There is a homogeneous symplectomorphism switching the roles of the variables in $V$ and $V^{*}$ (if $V$ is not already trivial); if $\eta_{1}^{\prime \prime}$ is away from 0 , as one may assume, one can take the map on $V \oplus W \oplus V^{*} \oplus W^{*}$ given by

$$
\left(y^{\prime}, y_{1}^{\prime \prime}, \eta^{\prime}, \eta_{1}^{\prime \prime}\right) \mapsto\left(-\frac{\eta^{\prime}}{\eta_{1}^{\prime \prime}}, y_{1}^{\prime \prime}+\frac{y^{\prime} \cdot \eta^{\prime}}{\eta_{1}^{\prime \prime}}, \eta_{1}^{\prime \prime} y^{\prime}, \eta_{1}^{\prime \prime}\right),
$$

with the rest of the $y^{\prime \prime}$ and $\eta^{\prime \prime}$ as well as the $z$ and $\zeta$ are unchanged under the map. Note that the pullback of the differentials of the new $\eta$ coordinates is $\eta_{1}^{\prime \prime} d y_{j}^{\prime}+y_{j}^{\prime} d \eta_{1}^{\prime \prime}$ and $d \eta_{j}^{\prime \prime}$, which have the same span as $d y_{j}^{\prime}$ and $d \eta_{j}^{\prime \prime}$, hence pull-back to be linearly independent in $T_{q}^{*} \mathcal{L}$. Thus, one can arrange that $\mathcal{L}$ is locally a graph over $\{0\} \times\left(\mathbb{R}^{n}\right)_{\eta, \zeta}^{*}$, which in addition is conic, so $Y$ and $Z$ as above are homogeneous of degree zero. Then $F$ may be arranged to be homogeneous of degree 1 (we can take $F=\sum \eta_{j} Y_{j}+\sum \zeta_{j} Z_{j}$-see [9, Proof of Theorem 21.2.16]), so (14.1) is homogeneous, proving the lemma.

Proposition 14.2. Let $p \in \mathcal{F}_{I}$ and let $q \in \mathcal{F}_{I}^{X}$ be its projection. Let $u$ be a solution to the wave equation with Cauchy data that is in $\mathcal{D}_{\infty}$ near $\partial X$ and that in $X^{\circ}$ is given by $\left.\left(u, D_{t} u\right)\right|_{t=0}=\left(u_{0}, u_{1}\right)$ with $\left(u_{0}, u_{1}\right) \in\left(I^{s}(\mathcal{L}), I^{s-1}(\mathcal{L})\right)$ where $\mathcal{L}$ is a Lagrangian intersecting $\mathcal{F}^{X}$ transversely at $\dot{\mathcal{F}}_{q}^{X}$. Then $u$ satisfies the nonfocusing condition of degree $(-s-n / 4)+f / 2-$, microlocally along $\dot{\mathcal{F}}_{I, p}$.

Note that $-s-n / 4-0$ is the a priori Sobolev regularity of the initial data, hence the gain in this result is of $f / 2-0$ derivatives.

Proof. Without loss of generality, we may take $u_{1}=0$ (if instead, $u_{0}=0$ we simply consider the solution $\partial_{t} u$ ). Decomposing by a partition of unity, it suffices to assume that $u_{0}$ has support in a small open set in $X^{\circ}$.

By the preceding lemma, there exists a local symplectomorphism $\Phi$ that reduces this geometric configuration to a normal form: $\Phi$ maps $\mathcal{F}^{X}$ to the set $\{\zeta=0\}$ and $\mathcal{L}$ to $N^{*}\{p\}$ with $p \in X^{\circ}$. Quantize $\Phi$ to an FIO $T$ of order 0 , with parametrix $S$, so that $S T-I, T S-I \in \Psi^{-\infty}\left(M^{\circ}\right)$. Thus if $Y \in \mathcal{A}^{m}$,

$$
T u=(T Y S) T v \Longrightarrow u=Y v+r, \quad r \in \mathcal{C}^{\infty}\left(M^{\circ}\right),
$$

and this would give the desired conclusion. As we are working locally in $M^{\circ}, T$ and $S$ preserve the scale of spaces $\mathcal{D}_{r}$; thus it now suffices to show that there exists $w \in \mathcal{D}_{-s-n / 4+f / 2-0}$ such that $\left(T(1+Y)^{m} S\right) w=T u$, with $Y \in \mathcal{A}^{2}$. By Egorov's theorem, TYS is a pseudodifferential operator of order 2 whose symbol vanishes quadratically on $\{\zeta=0\}$; it thus suffices to take $T Y S=\Delta_{z}$, where $\Delta_{z}$ is the (indeed, any) Laplacian in the fibers.

Meanwhile, $T u \in I^{s}\left(N^{*}\{p\}\right)$. We thus simply drop $T$ and take $u \in$ $I^{s}\left(N^{*}\{p\}\right), \mathcal{F}^{X}=\{\zeta=0\}, Y=\Delta_{z}$. We lump together the base variable $(x, y)$ to a single set of variables $\tilde{y}$, and shift our variables so that 
$\tilde{y}(p)=z(p)=0$. Thus, in our transformed coordinates we have

$$
(1+Y)^{-N} u=\int e^{i\left(\tilde{y} \cdot \eta+z^{\prime} \cdot \zeta^{\prime}+\left(z-z^{\prime}\right) \cdot \zeta\right)} a(z, \zeta) b\left(x, \tilde{y}, z^{\prime}, \xi, \eta, \zeta^{\prime}\right) d z^{\prime} d \xi d \eta d \zeta d \zeta^{\prime},
$$

where $a$, the symbol of $\left(1+\Delta_{z}\right)^{-N}$, is a symbol of order $-2 N$, and where $b$ is a symbol of order $s-n / 4$.

For $N$ sufficiently large, the integral in $\zeta$ is absolutely convergent, taking values in Lagrangian distributions of order $s+f / 4$ in the $\tilde{y}$ variables, hence

$$
(1+Y)^{-N} u \in \mathcal{C}\left(z ; H_{\text {loc }}^{-s-n / 4+f / 2-0}\right) \subset H_{\text {loc }}^{-s-n / 4+f / 2-0}\left(X^{\circ}\right) .
$$

Thus $u \in \mathcal{D}_{-s-n / 4+f / 2-0}+Y\left(\mathcal{D}_{-s-n / 4+f / 2-0}\right)+\cdots+Y^{N}\left(\mathcal{D}_{-s-n / 4+f / 2-0}\right)$.

\section{APPENDIX: ITERATIVE REGULARITY UNDER MODULES}

In this section we adapt the result of [7] and [8] to the present setting. The key new ingredient is that the set of radial points is not discrete, so we cannot be quite as specific with microlocalization as in these papers; we use the construction of Section 11. The notation below is that of Definition 4.2 and Lemma 4.4; additionally, we use the 'reduced' multiindex notation, dropping $A_{N}$, which is an elliptic multiple of $\square$ and thus treated separately, and set

$$
A_{\alpha}=\prod_{i=1}^{N-1} A_{i}^{\alpha_{i}}, \alpha_{i} \in \mathbb{N}_{0}, 1 \leq i \leq N-1 .
$$

Also let $W_{m, l} \in \Psi_{\mathrm{e}}^{m, l}(M)$ with $\sigma\left(W_{m, l}\right)=|\tau|^{m} x^{l}$, and let

$$
A_{\alpha, m, l}=W_{m, l} A_{\alpha} .
$$

The justification for treating $A_{N}$ separately is:

Lemma A.3. (See [7, Corollary 6.4].) Suppose $\mathcal{M}$ is a test module (see [7, Definition 6.1]), and $A_{N}$ is a generator with principal symbol $a_{N}$ an elliptic multiple of $\square$. Suppose $u \in \mathcal{C}^{-\infty}$ satisfies $\square u=0$ and $u$ is coisotropic of order $k-1$ on $O$ relative to $H_{\mathrm{e}}^{m, l}(M)$. Then for $O^{\prime} \subset O, u$ is coisotropic of order $k$ on $O^{\prime}$ relative to $H_{\mathrm{e}}^{m, l}(M)$ if for each multiindex $\alpha$, with $|\alpha|=k$, $\alpha_{N}=0$, there exists $Q_{\alpha} \in \Psi_{\mathrm{e}}^{0,0}(M)$, elliptic on $O^{\prime}$ such that $Q_{\alpha} A_{\alpha, m, l} u \in$ $L^{2}(M)$.

Proof. By Lemma 4.5 we only need to show that under our hypotheses, for $\alpha$ with $\alpha_{N} \neq 0$, there exists $Q_{\alpha} \in \Psi_{\mathrm{e}}^{0,0}(M)$ elliptic on $O^{\prime}$ such that $Q_{\alpha} A_{\alpha, m, l} u \in L^{2}(M)$. But such $A_{\alpha, m, l}$ is of the form $Q_{\alpha} A_{\beta, m, l} A_{N} u$ with $\beta_{j}=\alpha_{j}$ for $j \neq N, \beta_{N}=\alpha_{N}-1$, and $A_{N}=W_{-1,2} \square+B$, with $B \in \Psi_{\mathrm{e}}^{0,0}(M)$, $W_{-1,2} \in \Psi_{\mathrm{e}}^{-1,2}(M)$. By the hypotheses, $\square u=0$ while for any $Q_{\alpha} \in \Psi_{\mathrm{e}}^{0,0}(M)$ with $\mathrm{WF}_{\mathrm{e}}{ }^{\prime}\left(Q_{\alpha}\right) \subset O, Q_{\alpha} A_{\beta, m, l} B u \in L^{2}(M)$ since $u$ is coisotropic of order $k-1$ on $O$ relative to $H_{\mathrm{e}}^{m, l}(M)$ and $Q_{\alpha} A_{\beta, m, l} B \in \mathcal{A}^{k-1}$.

Thus, below $\alpha, \beta$ will stand for reduced multiindices, with $\alpha_{N}=0, \beta_{N}=0$. 
Lemma A.4. (Special case of [7, Lemma 6.5] adopted to the present setting.) Suppose $Q \in \Psi_{\mathrm{e}}^{0,0}(M)$, and let $C_{0}$ and $C_{i j}$ be given by (4.7), resp. (4.5). Then, assuming (4.5), and using the notation of (A.2), we have

(A.3)

$$
\begin{aligned}
& \sum_{|\alpha|=k} i\left[A_{\alpha, m, l}^{*} Q^{*} Q A_{\alpha, m, l}, \square\right] \\
& =\sum_{|\alpha|,|\beta|=k} A_{\alpha, m+1 / 2, l-1}^{*} Q^{*} C_{\alpha \beta}^{\prime} Q A_{\beta, m+1 / 2, l-1} \\
& \quad+\sum_{|\alpha|=k}\left(A_{\alpha, m+1 / 2, l-1}^{*} Q^{*} E_{\alpha, m+1 / 2, l-1}+E_{\alpha, m+1 / 2, l-1}^{*} Q A_{\alpha, m+1 / 2, l-1}\right) \\
& \quad+\sum_{|\alpha|=k} A_{\alpha, m, l}^{*} i\left[Q^{*} Q, \square\right] A_{\alpha, m, l},
\end{aligned}
$$

where

$$
E_{\alpha, m, l}=W_{m, l} E_{\alpha}, E_{\alpha} \in \mathcal{A}^{k-1}+\mathcal{A}^{k-1} A_{N}, \mathrm{WF}_{\mathrm{e}}{ }^{\prime}\left(E_{\alpha}\right) \subset \mathrm{WF}_{\mathrm{e}}{ }^{\prime}(Q),
$$

and for all $\alpha, \beta$,

$$
C_{\alpha \beta}^{\prime} \in \Psi_{\mathrm{e}}^{0,0}(M),\left.\sigma\left(C_{\alpha \beta}^{\prime}\right)\right|_{\dot{\mathcal{F}}}=-(m+l) \hat{\xi} \delta_{\alpha \beta},
$$

with $\delta$ denoting the Kronecker delta function.

Remark A.5. The first term on the right hand side of (A.3) is the principal term in terms of $\mathcal{A}$ order; both $A_{\alpha, m, l}$ and $A_{\beta, m, l}$ have $\mathcal{A}$ order $k$. Moreover, (A.4) states that it has non-negative principal symbol near $\partial \dot{\mathcal{F}}$. The terms involving $E_{\alpha, m, l}$ have $\mathcal{A}$ order $k-1$, or include a factor of $A_{N}$, so they can be treated as error terms. On the other hand, one does need to arrange that $i\left[Q^{*} Q, \square\right]$ is positive, as discussed below.

Proof. The commutator with $\square$ distributes over the factors in $A_{\alpha, m, l}=$ $W_{m, l} A_{1}^{\alpha_{1}} \ldots A_{N-1}^{\alpha_{N-1}}$. Using (4.5) for each individual commutator $i\left[A_{i}, \square\right]$, (4.7) for $W_{m, l}$, and rearranging the factors (with error, i.e. commutator, terms arising from rearrangement included in one of the $E_{\beta, m, l}$ terms as $\mathcal{M}$ is a Lie algebra) gives the conclusion. See [7, Proof of Lemma 6.5] for a more leisurely discussion.

We now consider the operators from Lemma A.4 $C^{\prime}=\left(C_{\alpha \beta}^{\prime}\right),|\alpha|=k=$ $|\beta|$, as a matrix of operators, or rather as an operator on a trivial vector bundle with fiber $\mathbb{R}^{\left|M_{k}\right|}$ over a neighborhood of $\dot{\mathcal{F}}$, where $\left|M_{k}\right|$ denotes the number of elements of the set $M_{k}$ of multiindices $\alpha$ with $|\alpha|=k$. Let $c^{\prime}=$ $\left.\sigma\left(C^{\prime}\right)\right|_{\partial \dot{\mathcal{F}}} \cdot$

Then $c^{\prime}=\left.\sigma\left(C^{\prime}\right)\right|_{\partial \dot{\mathcal{F}}}$ is positive or negative definite with the sign of $\left.\sigma\left(C_{0}\right)\right|_{\partial \dot{\mathcal{F}}}$. The same is therefore true microlocally near $\partial \dot{\mathcal{F}}$. For the sake of definiteness, suppose that $\sigma\left(C_{0}\right)_{\partial \dot{\mathcal{F}}}>0$. Then there exist a neighborhood 
$O_{k}$ of $\partial \dot{\mathcal{F}}$, depending on $|\alpha|=k$, and $B \in \Psi_{\mathrm{e}}^{0,0}(M), G \in \Psi_{\mathrm{e}}^{-1,0}(M)$, with $\sigma(B)>0$ on $O_{k}$ such that

$$
Q \in \Psi_{\mathrm{e}}^{m, l}(M), \mathrm{WF}_{\mathrm{e}}(Q) \subset O_{k} \Rightarrow Q^{*} C^{\prime} Q=Q^{*}\left(B^{*} B+G\right) Q .
$$

With $O_{k}$ as above, we assume that $\mathrm{WF}_{\mathrm{e}}{ }^{\prime}(Q) \subset O_{k}$,

$$
\begin{aligned}
& i\left[Q^{*} Q, \square\right]=\sum \tilde{B}_{j}^{*} \tilde{B}_{j}+\tilde{G}+\tilde{F}, \text { where } \\
& \tilde{B}_{j} \in \Psi_{\mathrm{e}}^{1 / 2,-1}(O), \tilde{F} \in \Psi_{\mathrm{e}}^{1,-2}(O), \tilde{G} \in \Psi_{\mathrm{e}}^{0,-2}(O) .
\end{aligned}
$$

In the actual application to solutions $u$ of the wave equation, stated below, $\tilde{F}$ will be such that $u$ is a priori regular on $\mathrm{WF}_{\mathrm{e}}{ }^{\prime}(\tilde{F})$, namely it is coisotropic of the order that we wish to propagate.

In fact, due to the two step nature of the proof below, we also need another microlocalizer $Q^{\prime} \in \Psi_{\mathrm{e}}^{0,0}(M)$ satisfying analogous assumptions with $\tilde{B}_{j}$, etc., replaced by $\tilde{B}_{j}^{\prime}$, etc.,

$$
i\left[\left(Q^{\prime}\right)^{*} Q^{\prime}, \square\right]=\sum\left(\tilde{B}_{j}^{\prime}\right)^{*} \tilde{B}_{j}^{\prime}+\tilde{G}^{\prime}+\tilde{F}^{\prime}
$$

with properties analogous to (A.6), except that $\mathrm{WF}_{\mathrm{e}}{ }^{\prime}\left(Q^{\prime}\right) \subset O_{k}^{\prime}$, etc., where $O_{k}^{\prime}$ is contained in the elliptic set of $Q$.

Proposition A.6. (Cf. [8, Appendix]; modified version of [7, Proposition 6.7].) Suppose that $\hat{\xi}<0$ on $O_{k}, u$ is coisotropic of order $k-1$ on $O_{k}$ relative to $H_{\mathrm{e}}^{m, l}(M), m>l+\frac{f}{2}, \mathrm{WF}_{\mathrm{e}}(\square u) \cap O_{k}=\emptyset$ and that there exist $Q, Q^{\prime} \in \Psi_{\mathrm{e}}^{0,0}\left(O_{k}\right)$ that satisfies (A.6)-(A.7) with u coisotropic of order $k$ on a neighborhood of $\mathrm{WF}_{\mathrm{e}}{ }^{\prime}(\tilde{F}) \cup \mathrm{WF}_{\mathrm{e}}{ }^{\prime}\left(\tilde{F}^{\prime}\right)$ relative to $H_{\mathrm{e}}^{m, l}(M)$. Then $u$ is coisotropic of order $k$ on $O^{\prime \prime}$ relative to $H_{\mathrm{e}}^{m, l}(M)$ where $O^{\prime \prime}$ is the elliptic set of $Q^{\prime}$.

The same conclusion holds if $\hat{\xi}>0$ on $O_{k}$ and $m<l+\frac{f}{2}$.

Proof. For the reader's convenience, we sketch the argument. We first prove a weaker statement, namely that $u$ is coisotropic of order $k$ on $O^{\prime}$ relative to $H_{\mathrm{e}}^{m-1 / 2, l}(M)$ where $O^{\prime}$ is the elliptic set of $Q$. Then we strengthen the result to complete the proof of the proposition.

First consider $u$ coisotropic of order $k$ on $O_{k}$ relative to $H_{\mathrm{e}}^{m, l}(M)$. Set

$$
r=m-1 \text { and } s=-l-\frac{f-1}{2} .
$$


Let $A u^{\prime}=\left(Q A_{\alpha, r+1 / 2, s-1} u^{\prime}\right)_{|\alpha|=k}$, regarded as a column vector of length $\left|M_{k}\right|$. Now consider

$$
\begin{aligned}
& \text { (A.8) } \sum_{|\alpha|=k}\left\langle u^{\prime}, i\left[A_{\alpha, r, s}^{*} Q^{*} Q A_{\alpha, r, s}, \square\right] u^{\prime}\right\rangle=\left\|B A u^{\prime}\right\|^{2}+\left\langle A u^{\prime}, G A u^{\prime}\right\rangle+ \\
& \sum_{|\alpha|=k}\left(\left\langle Q A_{\alpha, r+1 / 2, s-1} u^{\prime}, E_{\alpha, r+1 / 2, s-1} u^{\prime}\right\rangle+\left\langle E_{\alpha, r+1 / 2, s-1} u^{\prime}, Q A_{\alpha, r+1 / 2, s-1} u^{\prime}\right\rangle\right) \\
& +\sum_{|\alpha|=k}\left(\left\|\tilde{B} A_{\alpha, r, s} u^{\prime}\right\|^{2}+\left\langle A_{\alpha, r, s} u^{\prime}, \tilde{F} A_{\alpha, r, s} u^{\prime}\right\rangle+\left\langle A_{\alpha, r, s} u^{\prime}, \tilde{G} A_{\alpha, r, s} u^{\prime}\right\rangle\right) .
\end{aligned}
$$

Dropping the term involving $\tilde{B}$ and applying the Cauchy-Schwarz inequality to the terms with $E_{\alpha, r+1 / 2, s-1}$, we have for any $\epsilon>0$,

$$
\begin{aligned}
\left\|B A u^{\prime}\right\|^{2} \leq \sum_{\alpha} \mid & \left\langle u^{\prime}, i\left[A_{\alpha, r, s}^{*} Q^{*} Q A_{\alpha, r, s}, \square\right] u^{\prime}\right\rangle \mid+\epsilon\left\|A u^{\prime}\right\|^{2} \\
& +\epsilon^{-1} \sum_{\alpha}\left\|E_{\alpha, r+1 / 2, s-1} u^{\prime}\right\|^{2}+\left|\left\langle A u^{\prime}, G A u^{\prime}\right\rangle\right| \\
& +\sum_{|\alpha|=k}\left(\left|\left\langle A_{\alpha, r, s} u^{\prime}, \tilde{F} A_{\alpha, r, s} u^{\prime}\right\rangle\right|+\left|\left\langle A_{\alpha, r, s} u^{\prime}, \tilde{G} A_{\alpha, r, s} u^{\prime}\right\rangle\right|\right) .
\end{aligned}
$$

Choosing $\epsilon>0$ small enough, the second term on the right can be absorbed in the left hand side (since $B$ is strictly positive), and we get

$$
\begin{aligned}
\frac{1}{2}\left\|B A u^{\prime}\right\|^{2} \leq \sum_{\alpha} & \left|\left\langle u^{\prime}, i\left[A_{\alpha, r, s}^{*} Q^{*} Q A_{\alpha, r, s}, \square\right] u^{\prime}\right\rangle\right| \\
& +\epsilon^{-1} \sum_{\alpha}\left\|E_{\alpha, r+1 / 2, s-1} u^{\prime}\right\|^{2}+\left|\left\langle A u^{\prime}, G A u^{\prime}\right\rangle\right| \\
& +\sum_{|\alpha|=k}\left|\left\langle A_{\alpha, r, s} u^{\prime}, \tilde{G} A_{\alpha, r, s} u^{\prime}\right\rangle\right|+\sum_{|\alpha|=k}\left|\left\langle A_{\alpha, r, s} u^{\prime}, \tilde{F} A_{\alpha, r, s} u^{\prime}\right\rangle\right|
\end{aligned}
$$

Now, all but the first and last terms on the right hand side are bounded by the square of a coisotropic order $k-1$ norm of $u^{\prime}$ relative to $H_{\mathrm{e}}^{m, l}(M)$.

For the $E_{\alpha, r+1 / 2, s-1}$ term this is immediate, for $r+1 / 2=m-1 / 2 \leq m$, $s-1=-l-\frac{f+1}{2}$, and $E_{\alpha, r+1 / 2, s-1}=W_{r+1 / 2, s-1} E_{\alpha}, E_{\alpha} \in \mathcal{A}^{k-1}$, and as the pairing is relative to the Riemannian density $|d g|$, while the weighting of $H_{\mathrm{e}}^{m, l}(M)$ is relative to a smooth non-degenerate b-density, $\left\|E_{\alpha, r+1 / 2, s-1} u^{\prime}\right\|$ being bounded by a coisotropic order $k-1$ norm of $u$ relative to $H_{\mathrm{e}}^{m, l}(M)$ requires that $m-(r+1 / 2) \geq 0, l+\frac{f+1}{2}+s \geq 0$ (and these are $1 / 2$, resp. 0 ).

For the $G$ and $\tilde{G}$ terms, this can be seen by factoring out some $A_{j}$ from $A_{\alpha, r+1 / 2, s-1}=W_{r+1 / 2, s-1} A_{\alpha}$, i.e. writing it as $A_{\alpha, r+1 / 2, s-1}=A_{\beta, r+1 / 2, s-1} A_{j}$ with $|\beta|=|\alpha|-1=k-1$, and regarding $A_{j}$ simply as an operator in $\Psi_{\mathrm{e}}^{1,0}(M)$. As $G \in \Psi_{\mathrm{e}}^{-1,0}(M)$, the claimed boundedness requires $2 m-(-1+$ $2+2(r+1 / 2)) \geq 0,2 l+(f+1)+2(s-1) \geq 0$, and both are in fact 0 . For 
$\tilde{G} \in \Psi_{\mathrm{e}}^{0,-2}(M)$, the claimed boundedness requires (now we have factors of $\left.A_{\alpha, r, s}\right) 2 m-(0+2+2 r) \geq 0$ and $2 l+(f+1)+(-2+2 s) \geq 0$, and again both of these are 0 .

The first term on the right hand side vanishes if $\square u^{\prime}=0$, while the last term is bounded by the square of a coisotropic order $k$ norm of $u^{\prime}$ relative to $H_{\mathrm{e}}^{m, l}(M)$ in a neighborhood of $\mathrm{WF}_{\mathrm{e}}{ }^{\prime}(\tilde{F})$.

We apply this with $u^{\prime}$ replaced by $u_{\delta}=\left(1+\delta\left|D_{t}\right|\right)^{-1} u$ where now $u$ is coisotropic of order $k-1$ relative to $H_{\mathrm{e}}^{m, l}$ and still solves $\square u=0$. Then letting $\delta \rightarrow 0$, using the strong convergence of $\left(1+\delta\left|D_{t}\right|\right)^{-1}$ to the identity, and the assumption that $u$ is coisotropic of order $k$ relative to $H_{\mathrm{e}}^{m, l}(M)$ in a neighborhood of $\mathrm{WF}_{\mathrm{e}}{ }^{\prime}(\tilde{F})$, shows that $B A u \in L^{2}(M)$, hence that $u$ is coisotropic of order $k$ relative to $H_{\mathrm{e}}^{m-1 / 2, l}(M)$ on the elliptic set of $B$, finishing the proof of the first part.

For the second part, we carry through the same argument, but replacing $Q$ by $Q^{\prime}, \tilde{B}$ by $\tilde{B}^{\prime}$, etc., and taking

$$
r=m-\frac{1}{2}, s=-l-\frac{f-1}{2} .
$$

The claim is that all but the first and last terms on the right hand side of (A.9) (with the changes just mentioned) are bounded by the square of either a coisotropic norm of order $k-1$ of $u^{\prime}$ relative to $H_{\mathrm{e}}^{m, l}(M)$ (namely, the $E_{\alpha, r+1 / 2, s-1}$ term) or a coisotropic norm of order $k$ of $u^{\prime}$ relative to $H_{\mathrm{e}}^{m-1 / 2, l}(M)$ (the $G$ and $\tilde{G}^{\prime}$ terms).

For the $E_{\alpha, r+1 / 2, s-1}$ term, as above, the claimed boundedness requires $m-(r+1 / 2) \geq 0, l+\frac{f+1}{2}+s \geq 0$, and now these both vanish.

For the $G$ and $\tilde{G}^{\prime}$ terms the conclusion is also immediate, without factoring out an $A_{j}$ this time. Indeed, as $G \in \Psi_{\mathrm{e}}^{-1,0}(M)$, the claimed boundedness requires $2(m-1 / 2)-(-1+2(r+1 / 2)) \geq 0,2 l+(f+1)+2(s-1) \geq 0$, and both are in fact 0 . For $\tilde{G} \in \Psi_{\mathrm{e}}^{0,-2}(M)$, the claimed boundedness requires (now we have factors of $\left.A_{\alpha, r, s}\right) 2(m-1 / 2)-(0+2 r) \geq 0$ and $2 l+(f+1)+(-2+2 s) \geq 0$, and again both of these are 0 .

The regularization argument as above proves that $u$ is coisotropic of order $k$ relative to $H_{\mathrm{e}}^{m, l}(M)$ on the elliptic set of $B$, finishing the proof of the proposition.

\section{REFERENCES}

[1] V. A. Borovikov, The Green's function for a diffraction problem on a polyhedral angle. Dokl. Akad. Nauk SSSR 1511963 251-254.

[2] Jeff Cheeger and Michael Taylor, On the diffraction of waves by conical singularities. I, Comm. Pure Appl. Math. 35 (1982), no. 3, 275-331, MR84h:35091a.

[3] _ On the diffraction of waves by conical singularities. II, Comm. Pure Appl. Math. 35 (1982), no. 4, 487-529, MR84h:35091b.

[4] F. G. Friedlander, Sound pulses, Cambridge University Press, New York, 1958. MR 20 \#3703 
[5] F. G. Friedlander and R. B. Melrose, The wave front set of the solution of a simple initial-boundary value problem with glancing rays. II, Math. Proc. Cambridge Philos. Soc. 81 (1977), no. 1, 97-120. MR 55 \#10859

[6] Patrick Gérard and Gilles Lebeau, Diffusion d'une onde par un coin, J. Amer. Math. Soc. 6 (1993), no. 2, 341-424.

[7] Andrew Hassell, Richard Melrose, and András Vasy, Spectral and scattering theory for symbolic potentials of order zero, Adv. Math. 181 (2004), no. 1, 1-87. MR 2020655

[8] Andrew Hassell, Richard Melrose, and András Vasy, Scattering for symbolic potentials of order zero and microlocal propagation near radial points, preprint.

[9] L. Hörmander, The analysis of linear partial differential operators, vol. 3, SpringerVerlag, Berlin, Heidelberg, New York, Tokyo, 1985.

[10] J. Keller, One hundred years of diffraction theory, IEEE Trans. Antennas and Propagation 33 (1985), no. 2, 123-126.

[11] G. Lebeau, Propagation des ondes dans les variétés à coins, Séminaire sur les Équations aux Dérivées Partielles, 1995-1996, École Polytech., Palaiseau, 1996, MR98m:58137, pp. Exp. No. XVI, 20.

[12] Gilles Lebeau, Propagation des ondes dans les variétés à coins, Ann. Sci. École Norm. Sup. (4) 30 (1997), no. 4, 429-497, MR98d:58183.

[13] Rafe Mazzeo, Elliptic theory of differential edge operators. I, Comm. Partial Differential Equations 16 (1991), no. 10, 1615-1664. MR 93d:58152

[14] Richard B. Melrose, Microlocal parametrices for diffractive boundary value problems, Duke Math. J. 42 (1975), no. 4, 605-635. MR 58 \#24409

[15] _ Transformation of boundary problems, Acta Math. 147 (1981), no. 3-4, 149 236. MR 83f:58073

[16] Spectral and scattering theory for the Laplacian on asymptotically Euclidian spaces, Spectral and scattering theory (Sanda, 1992), Dekker, New York, 1994, pp. 85130. MR 95k:58168

[17] R. B. Melrose and J. Sjöstrand, Singularities of boundary value problems. I, Comm. Pure Appl. Math. 31 (1978), no. 5, 593-617. MR 58 \#11859.

[18] R. B. Melrose and J. Sjöstrand, Singularities of boundary value problems. II, Comm. Pure Appl. Math. 35 (1982), no. 2, 129-168. MR 83h:35120

[19] R. B. Melrose and J. Wunsch, Propagation of singularities for the wave equation on conic manifolds, Inventiones Mathematicae, 156 (2004), 235-299.

[20] Johannes Sjöstrand, Propagation of analytic singularities for second order Dirichlet problems, Comm. Partial Differential Equations 5 (1980), no. 1, 41-93. MR MR556454 (81e:35031a)

[21] _ Propagation of analytic singularities for second order Dirichlet problems. II, Comm. Partial Differential Equations 5 (1980), no. 2, 187-207. MR MR559155 (81e:35031b)

[22] _ Propagation of analytic singularities for second order Dirichlet problems. III, Comm. Partial Differential Equations 6 (1981), no. 5, 499-567. MR MR613851 (82k:35011)

[23] A. Sommerfeld, Mathematische theorie der diffraktion, Math. Annalen 47 (1896), $317-374$.

[24] M.E. Taylor, Grazing rays and reflection of singularities to wave equations, Comm. Pure Appl. Math. 29 (1978), 1-38.

[25] A. Vasy, Propagation of singularities for the wave equation on manifolds with corners, Ann. Math., to appear. 